TRANSACTIONS OF THE

AMERICAN MATHEMATICAL SOCIETY

Volume 363, Number 10, October 2011, Pages 5537-5576

S 0002-9947(2011)05310-9

Article electronically published on May 13, 2011

\title{
ON THE WEAK REFLECTION PRINCIPLE
}

\author{
JOHN KRUEGER
}

\begin{abstract}
The Weak Reflection Principle for $\omega_{2}$, or $\operatorname{WRP}\left(\omega_{2}\right)$, is the statement that every stationary subset of $P_{\omega_{1}}\left(\omega_{2}\right)$ reflects to an uncountable ordinal in $\omega_{2}$. The Reflection Principle for $\omega_{2}$, or $\operatorname{RP}\left(\omega_{2}\right)$, is the statement that every stationary subset of $P_{\omega_{1}}\left(\omega_{2}\right)$ reflects to an ordinal in $\omega_{2}$ with cofinality $\omega_{1}$. Let $\kappa$ be a $\kappa^{+}$-supercompact cardinal and assume $2^{\kappa}=\kappa^{+}$. Then there exists a forcing poset $\mathbb{P}$ which collapses $\kappa$ to become $\omega_{2}$, and $\Vdash_{\mathbb{P}} \operatorname{WRP}\left(\omega_{2}\right) \wedge \neg \operatorname{RP}\left(\omega_{2}\right)$.
\end{abstract}

In this paper we will be concerned with reflection of stationary subsets of $P_{\omega_{1}}\left(\omega_{2}\right)$. Recall that for an uncountable ordinal $\alpha$, a set $S \subseteq P_{\omega_{1}}(\alpha)=\left\{x \subseteq \alpha:|x|<\omega_{1}\right\}$ is stationary if for any function $F:[\alpha]^{<\omega} \rightarrow \alpha$, there is a set $b$ in $S$ which is closed under $F$. Let $S \subseteq P_{\omega_{1}}\left(\omega_{2}\right)$ be a stationary set. We say that $S$ reflects to $\alpha$, where $\alpha$ is an uncountable ordinal in $\omega_{2}$, if $S \cap P_{\omega_{1}}(\alpha)$ is stationary in $P_{\omega_{1}}(\alpha)$.

The Weak Reflection Principle for $\omega_{2}$, or $\operatorname{WRP}\left(\omega_{2}\right)$, is the statement that for every stationary set $S \subseteq P_{\omega_{1}}\left(\omega_{2}\right)$, there is an uncountable ordinal $\alpha$ in $\omega_{2}$ such that $S$ reflects to $\alpha$. This principle is a special case of the stronger Weak Reflection Principle introduced in [1]. The statement $\operatorname{WRP}\left(\omega_{2}\right)$ has a number of interesting combinatorial consequences, including $2^{\omega} \leq \omega_{2}$ ([4], [] $), \neg \square\left(\omega_{2}\right)$ ([7]), and every stationary subset of $\omega_{2} \cap \operatorname{cof}(\omega)$ reflects to an ordinal in $\omega_{2}$ with cofinality $\omega_{1}$.

A related principle is the Reflection Principle for $\omega_{2}$, or $\operatorname{RP}\left(\omega_{2}\right)$, which asserts that every stationary subset of $P_{\omega_{1}}\left(\omega_{2}\right)$ reflects to an ordinal in $\omega_{2}$ with cofinality $\omega_{1}$. The standard models for obtaining $\operatorname{WRP}\left(\omega_{2}\right)$ (for example, by Lévy collapsing a large cardinal to become $\left.\omega_{2}\right)$ all satisfy $\operatorname{RP}\left(\omega_{2}\right)$. Also, it tends to be easier to work with $\operatorname{RP}\left(\omega_{2}\right)$ than with $\operatorname{WRP}\left(\omega_{2}\right)$. Thus it is a natural question, and one which has been open for some time, whether $\operatorname{WRP}\left(\omega_{2}\right)$ implies $\operatorname{RP}\left(\omega_{2}\right)$.

A standard argument shows that if $\nabla_{A}$ holds for every stationary set $A \subseteq \omega_{2} \cap$ $\operatorname{cof}(\omega)$, then for any stationary set $S \subseteq P_{\omega_{1}}\left(\omega_{2}\right)$, there is a stationary set $T \subseteq S$ which does not reflect to any uncountable ordinal in $\omega_{2}$ with cofinality $\omega$. Therefore, assuming the existence of such diamonds, $\operatorname{WRP}\left(\omega_{2}\right)$ implies $\operatorname{RP}\left(\omega_{2}\right)$. Classically, the existence of such diamonds was known to follow from GCH; more recently, Shelah [5] has proven they are a consequence of $2^{\omega_{1}}=\omega_{2}$. Thus assuming $2^{\omega_{1}}=\omega_{2}$, $\mathrm{WRP}\left(\omega_{2}\right)$ implies $\mathrm{RP}\left(\omega_{2}\right)$ (originally, this result was proven in [2] by a different argument).

Received by the editors May 30, 2009 and, in revised form, February 8, 2010.

2010 Mathematics Subject Classification. Primary 03E35; Secondary $03 E 05$.

Key words and phrases. Weak Reflection Principle, Reflection Principle, generalized stationarity.

(C)2011 American Mathematical Society Reverts to public domain 28 years from publication 
The Weak Reflection Principle for $\omega_{2}$ is equiconsistent with a weakly compact cardinal. However, Sakai [3] has shown that a kind of local reflection is consistent from ZFC. Specifically, assuming GCH and $\square_{\omega_{1}}$, there is a generic extension in which there exists a stationary set $S \subseteq P_{\omega_{1}}\left(\omega_{2}\right)$ such that every stationary subset of $S$ reflects to an uncountable ordinal in $\omega_{2}$ with cofinality $\omega$.

We had the idea to combine parts of Sakai's argument with the classical methods for constructing models of the Weak Reflection Principle, in an attempt to show that $\operatorname{WRP}\left(\omega_{2}\right)$ does not imply $\operatorname{RP}\left(\omega_{2}\right)$. This paper is the culmination of those efforts. We will prove that if $\kappa$ is a $\kappa^{+}$-supercompact cardinal and $2^{\kappa}=\kappa^{+}$, then there is a forcing poset $\mathbb{P}$ which collapses $\kappa$ to become $\omega_{2}$ and forces $\operatorname{WRP}\left(\omega_{2}\right) \wedge \neg \operatorname{RP}\left(\omega_{2}\right)$.

\section{BACKGROUND MATERIAL}

We begin by recording some definitions and results which will be used in the paper.

By a forcing poset we mean an order which is reflexive and transitive (we do not assume it is antisymmetric or separative). Let $\mathbb{P}$ be a forcing poset, and let $\mu$ be a regular uncountable cardinal. We say $\mathbb{P}$ has the $\mu$-chain condition, or is $\mu$-c.c., if for any sequence $\left\langle p_{i}: i<\mu\right\rangle$ of conditions in $\mathbb{P}$ there are $i<j$ such that $p_{i}$ and $p_{j}$ are compatible. We say $\mathbb{P}$ is $\mu$-Knaster if for any sequence $\left\langle p_{i}: i<\mu\right\rangle$ of conditions in $\mathbb{P}$ there is a set $Z \subseteq \mu$ with $|Z|=\mu$ such that for all $i<j$ in $Z, p_{i}$ and $p_{j}$ are compatible. Clearly if $\mathbb{P}$ is $\mu$-Knaster, then $\mathbb{P}$ is $\mu$-c.c.

We say $\mathbb{P}$ is $\mu$-distributive if whenever $\left\langle D_{i}: i<\nu\right\rangle$ is a sequence of dense open subsets of $\mathbb{P}$, where $\nu<\mu$, then $\bigcap\left\{D_{i}: i<\nu\right\}$ is dense. This property implies that if $G$ is a generic filter on $\mathbb{P}$ over $V$, then for any set $x \subseteq V$ in $V[G]$ such that $V[G] \models|x|<\mu, x$ is in $V$. (Assuming $\mathbb{P}$ is separative, these two properties are equivalent, but they are not equivalent in general.)

A map $i: \mathbb{P} \rightarrow \mathbb{Q}$, where $\mathbb{P}$ and $\mathbb{Q}$ are forcing posets, is a complete embedding if:

(1) $q \leq p$ in $\mathbb{P}$ implies $i(q) \leq i(p)$ in $\mathbb{Q}$,

(2) if $i(p)$ and $i(q)$ are compatible in $\mathbb{Q}$, then $p$ and $q$ are compatible in $\mathbb{P}$,

(3) for all $q$ in $\mathbb{Q}$, there is $p$ in $\mathbb{P}$ such that whenever $t \leq p$ in $\mathbb{P}, i(t)$ and $q$ are compatible in $\mathbb{Q}$.

Suppose $i: \mathbb{P} \rightarrow \mathbb{Q}$ is a complete embedding. If $H$ is a generic filter on $\mathbb{Q}$ over $V$, then $i^{-1}(H)$ is a generic filter on $\mathbb{P}$ over $V$, and $V\left[i^{-1}(H)\right] \subseteq V[H]$. Note that if $\mathbb{P} \subseteq \mathbb{Q}$ and $i$ is the inclusion map, then $i^{-1}(H)=\{p \in \mathbb{P}: p \in H\}=H \cap \mathbb{P}$.

A complete embedding $i: \mathbb{P} \rightarrow \mathbb{Q}$ provides a way to translate $\mathbb{P}$-names to $\mathbb{Q}$ names. Define $i(\dot{x})$, for each $\mathbb{P}$-name $\dot{x}$, by recursion, letting $i(\dot{x})=\{(i(p), i(\dot{y}))$ : $(p, y) \in \dot{x}\}$. If $H$ is a generic filter on $\mathbb{Q}$ over $V$, then $i(\dot{x})^{H}=\dot{x}^{i^{-1}(H)}$. Note that if $i$ is the inclusion map, then for any $\mathbb{P}$-name $\dot{x}, i(\dot{x})=\dot{x}$. Therefore, if $H$ is a generic filter on $\mathbb{Q}$ over $V$, then $\dot{x}^{H}=\dot{x}^{H \cap \mathbb{P}}$.

A map $i: \mathbb{P} \rightarrow \mathbb{Q}$ is a dense embedding if

(1) $q \leq p$ in $\mathbb{P}$ implies $i(q) \leq i(p)$ in $\mathbb{Q}$,

(2) if $i(p)$ and $i(q)$ are compatible in $\mathbb{Q}$, then $p$ and $q$ are compatible in $\mathbb{P}$,

(3) $i[\mathbb{P}]$ is dense in $\mathbb{Q}$.

Every dense embedding is a complete embedding. If $i$ is a dense embedding, then $\mathbb{P}$ and $\mathbb{Q}$ are forcing equivalent in the sense that they have the same generic extensions. We will consider a particular kind of dense embedding, as follows. Say a map $i: \mathbb{P} \rightarrow \mathbb{Q}$ is an isomorphism if $i$ is a bijection, and $q \leq p$ in $\mathbb{P}$ iff $i(q) \leq i(p)$ in 
$\mathbb{Q}$. If $i: \mathbb{P} \rightarrow \mathbb{Q}$ is an isomorphism of $\mathbb{P}$ onto a dense subset of $\mathbb{Q}$, then $i$ is a dense embedding.

Let $\mathbb{P}$ and $\mathbb{Q}$ be forcing posets with maximum elements $1_{\mathbb{P}}$ and $1_{\mathbb{Q}}$, respectively. A map $\pi: \mathbb{Q} \rightarrow \mathbb{P}$ is a projection mapping if:

(1) $\pi\left(1_{\mathbb{Q}}\right)=1_{\mathbb{P}}$

(2) $q \leq p$ in $\mathbb{Q}$ implies $\pi(q) \leq \pi(p)$ in $\mathbb{P}$,

(3) if $t \leq \pi(q)$ in $\mathbb{P}$, then there is $r \leq q$ in $\mathbb{Q}$ such that $\pi(r) \leq t$ in $\mathbb{P}$.

Suppose $\pi: \mathbb{Q} \rightarrow \mathbb{P}$ is a projection mapping. If $H$ is a generic filter on $\mathbb{Q}$ over $V$, then $\pi[H]$ generates a generic filter on $\mathbb{P}$ over $V$. Furthermore, the projection mapping $\pi$ allows a factorization of $\mathbb{Q}$ into a two-step iteration $\mathbb{P} *(\mathbb{Q} / \mathbb{P})$ as follows. Let $G$ be a generic filter on $\mathbb{P}$ over $V$. In $V[G]$, define $\mathbb{Q} / \mathbb{P}$ as the forcing poset with underlying set $\{q \in \mathbb{Q}: \pi(q) \in G\}$, ordered as in $\mathbb{Q}$. Then the map $i: \mathbb{Q} \rightarrow \mathbb{P} *(\mathbb{Q} / \mathbb{P})$ defined by $i(q)=(\pi(q), \check{q})$ is an isomorphism of $\mathbb{Q}$ onto a dense subset of $\mathbb{P} *(\mathbb{Q} / \mathbb{P})$. Moreover, if $K$ is a generic filter on $\mathbb{Q}$ over $V$ and if $J$ is the filter on $\mathbb{P}$ generated by $\pi[K]$, then $K$ is a generic filter on $\mathbb{Q} / \mathbb{P}$ over $V[J]$, and $V[K]=V[J][K]$.

Lemma 1.1. Suppose $\pi: \mathbb{Q} \rightarrow \mathbb{P}$ is a projection mapping. If $D$ is a dense subset of $\mathbb{Q}$, then $\mathbb{P}$ forces $D \cap(\mathbb{Q} / \mathbb{P})$ is a dense subset of $\mathbb{Q} / \mathbb{P}$.

Proof. Let $G$ be a generic filter on $\mathbb{P}$ over $V$. Then $\mathbb{Q} / \mathbb{P}=\{q \in \mathbb{Q}: \pi(q) \in G\}$. Let $p$ be in $\mathbb{Q} / \mathbb{P}$, and we find $r \leq p$ in $D \cap(\mathbb{Q} / \mathbb{P})$. Note that $\pi(p) \in G$.

Let $D^{\prime}=\{u \leq \pi(p): \exists r \leq p(r \in D \wedge \pi(r)=u)\}$. We claim $D^{\prime}$ is dense below $\pi(p)$, so let $t \leq \pi(p)$ in $\mathbb{P}$ be given. By the definition of a projection mapping, fix $q \leq p$ in $\mathbb{Q}$ such that $\pi(q) \leq t$. Since $D$ is dense, choose $r \leq q$ in $D$. Let $u=\pi(r)$. Then $u=\pi(r) \leq \pi(q) \leq t$, so $u \leq t$. Also $r \leq q \leq p$, so $r \leq p, r \in D$, and $\pi(r)=u$, so $u \leq t$ and $u$ is in $D^{\prime}$.

Since $\pi(p)$ is in $G$ and $D^{\prime}$ is dense below $\pi(p)$, fix $u$ in $D^{\prime} \cap G$. Then there is $r \leq p$ in $D$ such that $\pi(r)=u$. Since $\pi(r)=u$ is in $G, r$ is in $D \cap(\mathbb{Q} / \mathbb{P})$.

Suppose $\mathbb{P}$ and $\mathbb{Q}$ are forcing posets. The Product Lemma says that for any set $G \subseteq \mathbb{P} \times \mathbb{Q}, G$ is a generic filter on $\mathbb{P} \times \mathbb{Q}$ over $V$ iff there is a generic filter $G_{\mathbb{P}}$ on $\mathbb{P}$ over $V$ and a generic filter $G_{\mathbb{Q}}$ on $\mathbb{Q}$ over $V\left[G_{\mathbb{P}}\right]$, such that $G=G_{\mathbb{P}} \times G_{\mathbb{Q}}$. Moreover, $V[G]=V\left[G_{\mathbb{P}}\right]\left[G_{\mathbb{Q}}\right]$, where $G=G_{\mathbb{P}} \times G_{\mathbb{Q}}$. Since $\mathbb{P} \times \mathbb{Q}$ is isomorphic to $\mathbb{Q} \times \mathbb{P}$ by the map $(p, q) \mapsto(q, p)$, it follows that if $G_{\mathbb{P}}$ is a generic filter on $\mathbb{P}$ over $V$ and $G_{\mathbb{Q}}$ is a generic filter on $\mathbb{Q}$ over $V\left[G_{\mathbb{P}}\right]$, then $G_{\mathbb{P}}$ is a generic filter on $\mathbb{P}$ over $V\left[G_{\mathbb{Q}}\right]$ and $V\left[G_{\mathbb{P}}\right]\left[G_{\mathbb{Q}}\right]=V\left[G_{\mathbb{Q}}\right]\left[G_{\mathbb{P}}\right]$.

We will apply the Product Lemma in the following way. Consider a generic extension $V[G][H]$, where $G$ is a generic filter on $\mathbb{P}$ over $V$ and $H$ is a generic filter on $\mathbb{Q}$ over $V[G]$. If it happens that the forcing poset $\mathbb{Q}$ is already in $V$, then by the Product Lemma, $V[G][H]=V[G \times H]=V[H \times G]=V[H][G]$. Therefore, $V[G][H]=V[H][G]$.

Let $X$ be a set. A nice $\mathbb{P}$-name for a subset of $X$ is a $\mathbb{P}$-name of the form $\bigcup\left\{A_{x} \times\{\check{x}\}: x \in X\right\}$, where for each $x$ in $X, A_{x}$ is an antichain of $\mathbb{P}$. A basic fact about $\mathbb{P}$-names is that if $p \Vdash \dot{A} \subseteq X$, then there is a nice $\mathbb{P}$-name $\dot{B}$ for a subset of $X$ such that $p \Vdash \dot{A}=\dot{B}$. Nice names are useful for controlling the continuum function. Let $Z$ be the collection of all nice $\mathbb{P}$-names for a subset of $X$. If $G$ is a generic filter on $\mathbb{P}$ over $V$, then in $V[G]$ the map $\dot{A} \mapsto \dot{A}^{G}$ is a surjective function from $Z$ onto $P(X)$. Hence $V[G] \models|P(X)| \leq|Z|$. Usually the chain condition and the size of $\mathbb{P}$ are used to put an upper bound on the size of $Z$. 
Suppose $f: X \rightarrow Y$ is a function and $a$ is a subset of $X$. If we say $a$ is closed under $f$, this implicitly assumes that $a$ is non-empty. If $f$ is a function and $A \subseteq \operatorname{dom}(f)$, we write $f[A]$ for the pointwise image of $A,\{f(a): a \in A\}$.

For any set $A$, let $P_{\omega_{1}}(A)=\left\{a \subseteq A:|a|<\omega_{1}\right\}$. Suppose $\omega_{1} \subseteq A$. A set $C \subseteq P_{\omega_{1}}(A)$ is club if (1) whenever $\left\langle a_{n}: n<\omega\right\rangle$ is a sequence of sets in $C$ such that $a_{n} \subseteq a_{n+1}$ for all $n<\omega$, then $\bigcup\left\{a_{n}: n<\omega\right\} \in C$, and (2) for all $b$ in $P_{\omega_{1}}(A)$, there is $c$ in $C$ with $b \subseteq c$. A set $S \subseteq P_{\omega_{1}}(A)$ is stationary if for every club set $C \subseteq P_{\omega_{1}}(A), S \cap C$ is non-empty.

A useful characterization of stationarity is given in terms of functions. A set $S \subseteq P_{\omega_{1}}(A)$ is stationary iff for any function $F:[A]^{<\omega} \rightarrow A$ there is a set $a$ in $S$ which is closed under $F$. Fodor's Lemma says that whenever $S \subseteq P_{\omega_{1}}(A)$ is stationary and $F: S \rightarrow A$ satisfies that $F(b) \in b$ for all $b$ in $S$, then there is a stationary set $S^{\prime} \subseteq S$ and $x$ in $A$ such that $F(a)=x$ for all $a \in S^{\prime}$.

When we say a cardinal $\theta$ is much larger than a set $X$, we mean that $P(P(P(X)))$ is in $H(\theta)$. We will frequently consider countable elementary substructures of $H(\theta)$, where $\theta \geq \omega_{2}$ is a regular cardinal. Let $N \prec H(\theta)$ be countable. We claim that for any uncountable ordinal $\nu$ in $N, N \cap \nu$ is not in $N$. For if $N \cap \nu$ is in $N$, then since $\nu \nsubseteq N \cap \nu$, let $\xi$ be the least ordinal in $\nu \backslash(N \cap \nu)$. Then by elementarity, $\xi$ is in $N$, which is a contradiction.

Let $N \prec H(\theta)$ be countable. Suppose $C \in N$ is a club subset of $P_{\omega_{1}}(A)$. Then $N \cap A \in C$. Suppose $\mathbb{P}$ is a forcing poset in $N$. If $G$ is a generic filter on $\mathbb{P}$ over $V$, then $N[G]=\left\{\dot{x}^{G}: \dot{x}\right.$ is a $\mathbb{P}$-name in $\left.N\right\}$ is an elementary substructure of $H(\theta)^{V[G]}$. Assume $\mathbb{P}$ preserves $\omega_{1}, \dot{C}$ is a $\mathbb{P}$-name in $N$ and $p$ forces $\dot{C}$ is club in $P_{\omega_{1}}(A)$. If $G$ is a generic filter on $\mathbb{P}$ over $V$ and $p \in G$, then $C=\dot{C}^{G}$ is in $N[G]$, and hence $N[G] \cap A \in C$.

A condition $q$ is $N$-generic (for $\mathbb{P}$ ) if $q \Vdash N[\dot{G}] \cap V=N$, where $\dot{G}$ is the canonical $\mathbb{P}$-name for the generic filter. Suppose $p \Vdash \dot{C}$ is club in $P_{\omega_{1}}(A)$, where $\dot{C} \in N$ and $p$ is $N$-generic. Then by the last paragraph, $p \Vdash N[\dot{G}] \cap A \in \dot{C}$. But since $p$ is $N$-generic, $p \Vdash N[\dot{G}] \cap A=N \cap A$; therefore, $p \Vdash N \cap A \in \dot{C}$. A descending sequence of conditions $\left\langle p_{n}: n<\omega\right\rangle$ in $\mathbb{P} \cap N$ is $N$-generic if for every dense open set $D \subseteq \mathbb{P}$ in $N$, there is $n<\omega$ such that $p_{n} \in D \cap N$. It is always possible to construct an $N$-generic sequence by simply enumerating the dense open subsets of $\mathbb{P}$ which are in $N$ in order type $\omega$ and by defining a descending sequence of conditions in $N \cap \mathbb{P}$ by recursion, meeting each dense open set in the enumeration. If $\left\langle p_{n}: n\langle\omega\rangle\right.$ is an $N$-generic sequence and $q \leq p_{n}$ for all $n<\omega$, then $q$ is an $N$-generic condition.

We will use the following special case of the $\Delta$-System Lemma. Assume $\mathrm{CH}$. Then for any sequence $\left\langle x_{i}: i<\omega_{2}\right\rangle$ of countable sets, there is a set $Z \subseteq \omega_{2}$ with $|Z|=\omega_{2}$ and a countable set $b$, such that for all $i<j$ in $Z, x_{i} \cap x_{j}=b$.

A cardinal $\kappa$ is $\lambda$-supercompact, where $\kappa \leq \lambda$, if there exists an elementary embedding $j: V \rightarrow M$ with critical point $\kappa$ such that $j(\kappa)>\lambda$ and $M^{\lambda} \subseteq M$.

Suppose $j: N \rightarrow M$ is an elementary embedding, where $N$ and $M$ are transitive inner models of ZFC. Suppose $\mathbb{P}$ is a forcing poset in $N, G$ is a generic filter on $\mathbb{P}$ over $N$, and $H$ is a generic filter on $j(\mathbb{P})$ over $M$. Then $j$ can be extended to $j: N[G] \rightarrow M[H]$ such that $j(G)=H$ iff $j[G] \subseteq H$ (namely, let $j\left(\dot{x}^{G}\right)=j(\dot{x})^{H}$ ).

Let $M \subseteq N$ be inner models of ZFC, suppose $\lambda$ is a regular uncountable cardinal of $N$, and assume $M^{<\lambda} \cap N \subseteq M$. Let $\mathbb{P}$ be a forcing poset in $M$, and assume $N$ models that $\mathbb{P}$ is $\lambda$-c.c. If $G$ is a generic filter on $\mathbb{P}$ over $N$, then $M[G]<\lambda \cap N[G] \subseteq$ $M[G]$. 


\section{OUtLine OF THE PROOF}

The objective of this paper is to define a forcing poset $\mathbb{P}$ such that

$$
\Vdash_{\mathbb{P}} \operatorname{WRP}\left(\omega_{2}\right) \wedge \neg \mathrm{RP}\left(\omega_{2}\right) .
$$

Specifically, we will define a forcing poset $\mathbb{P}$ which satisfies the fact that whenever $K$ is a generic filter on $\mathbb{P}$ over $V$, then in $V[K]$ there is a set $S$ satisfying:

(1) $S$ is a stationary subset of $P_{\omega_{1}}\left(\omega_{2}\right)$,

(2) $S$ does not reflect to any ordinal in $\omega_{2} \cap \operatorname{cof}\left(\omega_{1}\right)$,

(3) every stationary subset of $S$ reflects to an uncountable ordinal in $\omega_{2} \cap \operatorname{cof}(\omega)$,

(4) every stationary subset of $P_{\omega_{1}}\left(\omega_{2}\right) \backslash S$ reflects to an ordinal in $\omega_{2} \cap \operatorname{cof}\left(\omega_{1}\right)$. Clearly statements (1) and (2) imply that $\neg R P\left(\omega_{2}\right)$ holds in $V[K]$. Statements (3) and (4) imply that $\operatorname{WRP}\left(\omega_{2}\right)$ holds in $V[K]$. For if $U$ is a stationary subset of $P_{\omega_{1}}\left(\omega_{2}\right)$, then either $U \cap S$ is stationary or $U \backslash S$ is stationary. In the first case, (3) implies there is an uncountable ordinal $\alpha$ in $\omega_{2} \cap \operatorname{cof}(\omega)$ such that $U \cap S$ reflects to $\alpha$. In the second case, (4) implies there is an ordinal $\alpha$ in $\omega_{2} \cap \operatorname{cof}\left(\omega_{1}\right)$ such that $U \backslash S$ reflects to $\alpha$. In either case, there is an uncountable ordinal $\alpha$ in $\omega_{2}$ such that $U$ reflects to $\alpha$.

We will define $\mathbb{P}$ in a model $V$ in which there is a cardinal $\kappa$ which is $\kappa^{+}$supercompact and $2^{\kappa}=\kappa^{+}$. The forcing poset $\mathbb{P}$ will be of the form

$$
\operatorname{CoLL}\left(\omega_{1},<\kappa\right) * \dot{\mathbb{P}}_{\omega_{2}} * \dot{\mathbb{Q}}
$$

Therefore, in the first step, we Lévy collapse $\kappa$ to become $\omega_{2}$. Let $G$ be a generic filter on $\operatorname{CoLL}\left(\omega_{1},<\kappa\right)$ over $V$. Note that in $V[G], 2^{\omega}=\omega_{1}, 2^{\omega_{1}}=\omega_{2}$, and $2^{\omega_{2}}=\omega_{3}$.

In $V[G]$ we define a forcing poset $\mathbb{P}_{\omega_{2}}$ which adds a generic stationary set $S \subseteq$ $P_{\omega_{1}}\left(\omega_{2}\right)$ which does not reflect to any ordinal in $\omega_{2} \cap \operatorname{cof}\left(\omega_{1}\right)$.

Definition 2.1. Let $\mathbb{P}_{\omega_{2}}$ be the forcing poset consisting of pairs $(X, F)$ satisfying:

(1) $X$ is a countable subset of $P_{\omega_{1}}\left(\omega_{2}\right) \backslash\{\emptyset\}$,

(2) $F$ is a function whose domain is a countable subset of $\omega_{2} \cap \operatorname{cof}\left(\omega_{1}\right)$,

(3) for all $\xi$ in $\operatorname{dom}(F), F(\xi)$ is a $\subseteq$-increasing and continuous sequence $\left\langle a_{i}^{\xi}\right.$ : $\left.i \leq \gamma_{\xi}\right\rangle$ of countable subsets of $\xi$, for some $\gamma_{\xi}<\omega_{1}$,

(4) for all $\xi$ in $\operatorname{dom}(F)$ and $i \leq \gamma_{\xi}, a_{i}^{\xi}$ is not in $X$,

(5) for all $\xi$ in $\operatorname{dom}(F), a_{\gamma_{\xi}}^{\xi} \subseteq \bigcup X$.

Define an order on $\mathbb{P}_{\omega_{2}}$ by letting $(Y, K) \leq(X, F)$ if:

(a) $X \subseteq Y$,

(b) for all $b$ in $Y \backslash X, b \nsubseteq \bigcup X$,

(c) $\operatorname{dom}(F) \subseteq \operatorname{dom}(K)$,

(d) for all $\xi$ in $\operatorname{dom}(F), F(\xi)$ is an initial segment of $K(\xi)$.

The forcing poset $\mathbb{P}_{\omega_{2}}$ is $\omega_{1}$-distributive. Since $2^{\omega}=\omega_{1}$ holds in $V[G], \mathbb{P}_{\omega_{2}}$ is $\omega_{2}$-c.c. It is easy to see that $\mathbb{P}_{\omega_{2}}$ forces $2^{\omega}=\omega_{1}, 2^{\omega_{1}}=\omega_{2}$, and $2^{\omega_{2}}=\omega_{3}$.

Let $H$ be a generic filter on $\mathbb{P}_{\omega_{2}}$ over $V$. Define $S=\bigcup\{X: \exists F(X, F) \in H\}$. Then $S$ is a stationary subset of $P_{\omega_{1}}\left(\omega_{2}\right)$. For each $\nu$ in $\omega_{2} \cap \operatorname{cof}\left(\omega_{1}\right)$, the union of the collection of sequences $\{F(\nu): \exists X(X, F) \in H, \nu \in \operatorname{dom}(F)\}$ is an increasing and continuous sequence $\left\langle a_{i}^{\nu}: i<\omega_{1}\right\rangle$, with union equal to $\nu$, which is disjoint from $S$. It follows that $S$ does not reflect to any ordinal in $\omega_{2} \cap \operatorname{cof}\left(\omega_{1}\right)$. Moreover, by 
upwards absoluteness, $S$ does not reflect to any ordinal in $\omega_{2} \cap \operatorname{cof}\left(\omega_{1}\right)$ in any outer model of $V[G * H]$ with the same $\omega_{1}$ and $\omega_{2}$.

In $V[G * H]$, we define a forcing iteration $\mathbb{Q}$, which is designed to destroy the stationarity of any subset of $S$ which does not reflect to an uncountable ordinal in $\omega_{2} \cap \operatorname{cof}(\omega)$. This forcing poset is $\omega_{1}$-distributive and $\omega_{2}$-c.c. in $V[G * H]$. Let $I$ be a generic filter on $\mathbb{Q}$ over $V[G * H]$. Then in $V[G * H * I], S$ is a stationary subset of $P_{\omega_{1}}\left(\omega_{2}\right)$ which does not reflect to any ordinal in $\omega_{2} \cap \operatorname{cof}\left(\omega_{1}\right)$, and moreover, every stationary subset of $S$ reflects to an uncountable ordinal in $\omega_{2} \cap \operatorname{cof}(\omega)$.

Finally, let $K=G * H * I$. Statements (1), (2), and (3) hold in $V[K]$, as we have already observed. It remains to show (4), where every stationary subset of $P_{\omega_{1}}\left(\omega_{2}\right) \backslash S$ reflects to an ordinal in $\omega_{2} \cap \operatorname{cof}\left(\omega_{1}\right)$.

In the ground model $V$, let $j: V \rightarrow M$ be an elementary embedding with critical point $\kappa$ such that $j(\kappa)>\kappa^{+}$and $M^{\kappa^{+}} \subseteq M$. Let $\bar{G} * \bar{H} * \bar{I}$ be a generic filter on $j\left(\operatorname{Coll}\left(\omega_{1},<\kappa\right) * \dot{\mathbb{P}}_{\omega_{2}} * \dot{\mathbb{Q}}\right)$ over $V$. Let $G=\bar{G} \cap \operatorname{ColL}\left(\omega_{1},<\kappa\right)$ and $H=\bar{H} \cap \mathbb{P}_{\omega_{2}}$. Then $G$ is a generic filter on $\operatorname{ColL}\left(\omega_{1},<\kappa\right)$ over $V$, and $H$ is a generic filter on $\mathbb{P}_{\omega_{2}}$ over $V[G]$. In $V[\bar{G} * \bar{H}]$, we can extend $j$ to $j: V[G * H] \rightarrow M[\bar{G} * \bar{H}]$ such that $j(G * H)=\bar{G} * \bar{H}$. Also let $G^{\prime}=\bar{G} \cap \operatorname{CoLL}\left(\omega_{1},[\kappa, j(\kappa))\right)$. Note that $M[\bar{G}]=M[G]\left[G^{\prime}\right]$.

In the model $M[\bar{G}], j\left(\mathbb{P}_{\omega_{2}}\right)$ factors as a two-step iteration

$$
\mathbb{P}_{\omega_{2}} * \mathbb{P}(\kappa, S, j(\kappa))
$$

(The definition of $\mathbb{P}(\kappa, S, j(\kappa))$ will be given in the next section; it is a generalization of Definition 2.1.) Since $j\left(\mathbb{P}_{\omega_{2}}\right)$ is forcing equivalent to $\mathbb{P}_{\omega_{2}} * \mathbb{P}(\kappa, S, j(\kappa))$, there exists a generic filter $H^{\prime}$ on $\mathbb{P}(\kappa, S, j(\kappa))$ over $M[\bar{G}][H]$ such that $M[\bar{G}][\bar{H}]=$ $M[\bar{G}][H]\left[H^{\prime}\right]$.

In the model $M[\bar{G} * \bar{H}]$, there exists a projection mapping $\pi: j(\mathbb{Q}) \rightarrow \mathbb{Q}$. This allows us to factor $j(\mathbb{Q})$ as $\mathbb{Q} *(j(\mathbb{Q}) / \mathbb{Q})$. Let $I$ be the filter on $\mathbb{Q}$ generated by $\pi[\bar{I}]$. Then $M[\bar{G} * \bar{H}][\bar{I}]=M[\bar{G} * \bar{H}][I][\bar{I}]$. In the model $V[\bar{G} * \bar{H} * \bar{I}]$, we extend $j$ to $j: V[G * H * I] \rightarrow M[\bar{G} * \bar{H} * \bar{I}]$ such that $j(G * H * I)=\bar{G} * \bar{H} * \bar{I}$. By several applications of the Product Lemma, the model $M[\bar{G} * \bar{H} * \bar{I}]$ can be shown to be equal to $M[G * H * I]\left[G^{\prime}\right]\left[H^{\prime}\right][\bar{I}]$.

Suppose $T$ is a stationary subset of $P_{\omega_{1}}\left(\omega_{2}\right) \backslash S$ in the model $V[G * H * I]$. Since the forcing poset $\operatorname{CoLL}\left(\omega_{1},<\kappa\right) * \dot{\mathbb{P}}_{\omega_{2}} * \dot{\mathbb{Q}}$ is $\kappa$-c.c., $M[G * H * I]^{\kappa^{+}} \cap V[G * H * I] \subseteq$ $M[G * H * I]$. Therefore, in $M[G * H * I], T$ is a stationary subset of $P_{\omega_{1}}(\kappa)$. The model $M[\bar{G} * \bar{H} * \bar{I}]$ is a generic extension of $M[G * H * I]$ by the forcing poset $\operatorname{CoLL}\left(\omega_{1},[\kappa, j(\kappa))\right) * \mathbb{P}(\kappa, S, j(\kappa)) *(j(\mathbb{Q}) / \mathbb{Q})$. It turns out that this forcing poset preserves the stationarity of any stationary subset of $P_{\omega_{1}}(\kappa)$ in $M[G * H * I]$ which is disjoint from $S$. Thus $T$ remains stationary in $M[\bar{G} * \bar{H} * \bar{I}]$.

In the model $M[\bar{G} * \bar{H} * \bar{I}], j(T)$ reflects to $\kappa$, since $j(T) \cap P_{\omega_{1}}(\kappa)=T$ and $T$ is stationary in $P_{\omega_{1}}(\kappa)$. In this model, $\kappa$ is an ordinal in $j(\kappa)=\omega_{2}$ with cofinality $\omega_{1}$. By the elementarity of $j$, in $V[G * H * I]$ the set $T$ reflects to an ordinal in $\omega_{2}$ with cofinality $\omega_{1}$.

\section{The Forcing poset $\mathbb{P}(\alpha, A, \beta)$}

In the previous section we defined the forcing poset $\mathbb{P}_{\omega_{2}}$, which adds a stationary subset of $P_{\omega_{1}}\left(\omega_{2}\right)$ which does not reflect to any ordinal in $\omega_{2}$ with cofinality $\omega_{1}$. For the purpose of extending an elementary embedding after forcing with $\mathbb{P}_{\omega_{2}}$, we will need to consider a more general version of this forcing poset. 
Definition 3.1. Let $\alpha<\beta \leq \omega_{2}$ be ordinals, where $\beta$ has uncountable cofinality. Let $A$ be a subset of $P_{\omega_{1}}(\alpha)$. If $\operatorname{cf}(\alpha)=\omega_{1}$, also assume $P_{\omega_{1}}(\alpha) \backslash A$ is stationary in $P_{\omega_{1}}(\alpha)$. Define a forcing poset $\mathbb{P}(\alpha, A, \beta)$ as follows. A condition in $\mathbb{P}(\alpha, A, \beta)$ is a pair $(X, F)$ satisfying:

(1) $X$ is a countable subset of $P_{\omega_{1}}(\beta)$,

(2) for all $b$ in $X, b$ is not a subset of $\alpha$,

(3) $F$ is a function whose domain is a countable subset of $[\alpha, \beta) \cap \operatorname{cof}\left(\omega_{1}\right)$,

(4) for all $\xi$ in $\operatorname{dom}(F), F(\xi)$ is a $\subseteq$-increasing and continuous sequence $\left\langle a_{i}^{\xi}\right.$ : $\left.i \leq \gamma_{\xi}\right\rangle$ of countable subsets of $\xi$, for some $\gamma_{\xi}<\omega_{1}$,

(5) for all $\xi$ in $\operatorname{dom}(F)$ and $i \leq \gamma_{\xi}, a_{i}^{\xi}$ is not in $X \cup A$,

(6) for all $\xi$ in $\operatorname{dom}(F), a_{\gamma_{\xi}}^{\xi} \subseteq \bigcup X$.

Define an order on $\mathbb{P}(\alpha, A, \beta)$ by letting $(Y, K) \leq(X, F)$ if:

(a) $X \subseteq Y$,

(b) for all $b$ in $Y \backslash X, b \nsubseteq \bigcup X$,

(c) $\operatorname{dom}(F) \subseteq \operatorname{dom}(K)$,

(d) for all $\xi$ in $\operatorname{dom}(F), F(\xi)$ is an initial segment of $K(\xi)$.

Note that property (2) implies every set in $X$ is non-empty. Also, for any condition $(X, F)$ in $\mathbb{P}(\alpha, A, \beta), X \cap A$ is empty. Namely, if $b$ is in $X$, then $b$ is not a subset of $\alpha$ by (2). However, $A$ is a subset of $P_{\omega_{1}}(\alpha)$.

If $(X, F)$ is a condition, then $X$ is an approximation of a generic stationary set $S \subseteq P_{\omega_{1}}(\beta)$. The function $F$ in $(X, F)$ approximates an array of clubs which witnesses that $S$ does not reflect to any ordinal in $[\alpha, \beta)$ with cofinality $\omega_{1}$.

We will only be interested in two particular cases of the definition. The first case occurs when $\alpha=0$ and $A=\emptyset$. In this case, we abbreviate $\mathbb{P}(\alpha, A, \beta)$ as $\mathbb{P}_{\beta}$. For example, the forcing poset $\mathbb{P}_{\omega_{2}}$ described in the previous section is equal to $\mathbb{P}\left(0, \emptyset, \omega_{2}\right)$.

The second case of interest occurs in the following context. Let $\nu<\omega_{2}$ be an ordinal with cofinality $\omega_{1}$. Let $H$ be a generic filter on $\mathbb{P}_{\nu}$ over $V$. In $V[H]$, define $A=\bigcup\{X: \exists F(X, F) \in H\}$. Therefore, $A$ is a subset of $P_{\omega_{1}}(\nu)$. As we will prove later, $P_{\omega_{1}}(\nu) \backslash A$ is stationary in $P_{\omega_{1}}(\nu)$. It is meaningful to define $\mathbb{P}\left(\nu, A, \omega_{2}\right)$ in the model $V[H]$. As it turns out, $\mathbb{P}_{\omega_{2}}$ is forcing equivalent to the two-step iteration $\mathbb{P}_{\nu} * \mathbb{P}\left(\nu, A, \omega_{2}\right)$. This factorization is the motivation for Definition 3.1.

Notation 3.2. Let $\beta \leq \omega_{2}$ be an ordinal with uncountable cofinality. Then $\mathbb{P}_{\beta}$ denotes $\mathbb{P}(0, \emptyset, \beta)$.

The proof of the next lemma is straightforward.

Lemma 3.3. Let $\beta_{1}<\beta_{2} \leq \omega_{2}$ be ordinals with uncountable cofinality. Then $\mathbb{P}_{\beta_{1}} \subseteq \mathbb{P}_{\beta_{2}}$. If $p$ and $q$ are in $\mathbb{P}_{\beta_{1}}$, then $q \leq p$ in $\mathbb{P}_{\beta_{1}}$ iff $q \leq p$ in $\mathbb{P}_{\beta_{2}}$.

Lemma 3.4. Let $p=(X, F)$ be a condition in $\mathbb{P}(\alpha, A, \beta)$, and let $d$ be a set in $P_{\omega_{1}}(\beta)$ such that $d$ is not a subset of $\alpha$ and $d$ is not a subset of $\bigcup X$. Then $q=(X \cup\{d\}, F)$ is a condition in $\mathbb{P}(\alpha, A, \beta)$ and $q \leq p$.

Proof. Properties (1), (2), (3), (4), and (6) of Definition 3.1 are immediate. For (5), let $\xi$ be in $\operatorname{dom}(F)$ and let $F(\xi)=\left\langle a_{i}^{\xi}: i \leq \gamma_{\xi}\right\rangle$. Let $i \leq \gamma_{\xi}$. Then $a_{i}^{\xi}$ is not in $X \cup A$. Also $a_{i}^{\xi}$ is a subset of $\bigcup X$. Since $d$ is not a subset of $\bigcup X, a_{i}^{\xi}$ is not equal to $d$. Therefore, $a_{i}^{\xi}$ is not in $(X \cup\{d\}) \cup A$. It is immediate that $q \leq p$. 
Lemma 3.5. Let $b$ be a set in $P_{\omega_{1}}(\beta)$. Then there are densely many conditions $(Y, K)$ in $\mathbb{P}(\alpha, A, \beta)$ such that $b \subseteq \bigcup Y$.

Proof. Let $(X, F)$ be a condition. Choose a countable set $d \subseteq \beta$ such that $b \cup$ $(\cup X) \cup\{\alpha\} \subsetneq d$. By Lemma 3.4, $(X \cup\{d\}, F)$ is a condition in $\mathbb{P}(\alpha, A, \beta)$ which is below $(X, F)$, and clearly $b \subseteq \bigcup(X \cup\{d\})$.

Lemma 3.6. Let $\xi$ be an ordinal in $[\alpha, \beta) \cap \operatorname{cof}\left(\omega_{1}\right)$, let $\pi<\omega_{1}$, and let $b$ be a countable subset of $\xi$. Then there are densely many conditions in $\mathbb{P}(\alpha, A, \beta)$ of the form $(Y, K)$, where $\xi$ is in $\operatorname{dom}(K)$ and $K(\xi)=\left\langle b_{i}^{\xi}: i \leq \gamma_{\xi}\right\rangle$, where $\pi \leq \gamma_{\xi}$ and $b \subseteq b_{\gamma_{\xi}}^{\xi}$.

Proof. Let $(X, F)$ be a condition in $\mathbb{P}(\alpha, A, \beta)$. We choose sets $c$ and $d$ as follows, depending on whether $\xi>\alpha$ or $\xi=\alpha$. First suppose $\xi>\alpha$. Since $\xi$ is uncountable and $\bigcup X$ is countable, we can choose countable sets $c$ and $d$ such that $((\bigcup X) \cap \xi) \cup b \cup$ $\{\alpha\} \varsubsetneqq c \varsubsetneqq d \subseteq \xi$. Now suppose $\xi=\alpha$. Then $\operatorname{cf}(\alpha)=\omega_{1}$, so $P_{\omega_{1}}(\alpha) \backslash A$ is stationary in $P_{\omega_{1}}(\alpha)$. Therefore, we can choose $c$ in $P_{\omega_{1}}(\alpha) \backslash A$ such that $((\bigcup X) \cap \alpha) \cup b \varsubsetneqq c$. Now let $d=c \cup\{\alpha\}$.

Define $Y=X \cup\{d\}$. By the choice of $d, \alpha \in d$ and $d \nsubseteq \subseteq \cup$. Therefore, by Lemma 3.4, $(Y, F)$ is a condition in $\mathbb{P}(\alpha, A, \beta)$ which is below $(X, F)$.

Now we define a function $K$. Let $K$ have domain equal to $\operatorname{dom}(F) \cup\{\xi\}$. For all $\nu$ in $\operatorname{dom}(K)$ different from $\xi$, let $K(\nu)=F(\nu)$. To define $K(\xi)$, we consider the two cases of whether or not $\xi$ is in $\operatorname{dom}(F)$. Suppose $\xi$ is not in $\operatorname{dom}(F)$. Then let $K(\xi)$ be the sequence $\left\langle b_{i}^{\xi}: i \leq \pi\right\rangle$, where $b_{i}^{\xi}=c$ for all $i \leq \pi$. Suppose $\xi$ is in $\operatorname{dom}(F)$. Let $F(\xi)=\left\langle a_{i}^{\xi}: i \leq \bar{\gamma}_{\xi}\right\rangle$ and let $\gamma_{\xi}=\max \left(\bar{\gamma}_{\xi}, \pi\right)+1$. Now define $K(\xi)=\left\langle b_{i}^{\xi}: i \leq \gamma_{\xi}\right\rangle$, where $b_{i}^{\xi}=a_{i}^{\xi}$ for $i \leq \bar{\gamma}_{\xi}$ and $b_{j}^{\xi}=c$ for $\bar{\gamma}_{\xi}<j \leq \gamma_{\xi}$. It is routine to verify that $(Y, K)$ is a condition in $\mathbb{P}(\alpha, A, \beta)$ which is below $(X, F)$, and $(Y, K)$ clearly satisfies the conclusion of the lemma.

We will frequently consider descending sequences of conditions in $\mathbb{P}(\alpha, A, \beta)$. Therefore, it will be useful to establish some notation and basic facts about such sequences.

Suppose $\left\langle\left(X_{n}, F_{n}\right): n<\omega\right\rangle$ is a descending sequence of conditions in $\mathbb{P}(\alpha, A, \beta)$. Let us define what we will call the infinum of the sequence of functions $\left\langle F_{n}: n<\omega\right\rangle$. Let $K$ have domain equal to $\bigcup\left\{\operatorname{dom}\left(F_{n}\right): n<\omega\right\}$, which is a countable subset of $[\alpha, \beta) \cap \operatorname{cof}\left(\omega_{1}\right)$. Consider $\xi$ in the domain of $K$. If $n<m$ and $\xi$ is in the domain of $F_{n}$, then since $\left(X_{m}, F_{m}\right) \leq\left(X_{n}, F_{n}\right), F_{n}(\xi)$ is an initial segment of $F_{m}(\xi)$. If we take the union of all sequences $F_{n}(\xi)$, where $\xi \in \operatorname{dom}\left(F_{n}\right)$, we obtain an increasing and continuous sequence of the form $\left\langle a_{i}^{\xi}: i<\gamma_{\xi}\right\rangle$, where $\gamma_{\xi}<\omega_{1}$. If $\gamma_{\xi}$ is a successor ordinal, let $K(\xi)$ be the sequence $\left\langle a_{i}^{\xi}: i \leq \gamma_{\xi}-1\right\rangle$. If $\gamma_{\xi}$ is a limit ordinal, define $a_{\gamma_{\xi}}^{\xi}=\bigcup\left\{a_{i}^{\xi}: i<\gamma_{\xi}\right\}$, and let $K(\xi)=\left\langle a_{i}^{\xi}: i \leq \gamma_{\xi}\right\rangle$. Then $K(\xi)$ is an increasing and continuous sequence of countable subsets of $\xi$. We refer to $K$ as the infinum of the sequence $\left\langle F_{n}: n<\omega\right\rangle$.

Lemma 3.7. Let $\theta$ be a regular cardinal much larger than $\mathbb{P}(\alpha, A, \beta)$, and suppose $N$ is a countable elementary substructure of $H(\theta)$ such that $\mathbb{P}(\alpha, A, \beta)$ is in $N$. Let $\left\langle\left(X_{n}, F_{n}\right): n<\omega\right\rangle$ be an $N$-generic sequence for $\mathbb{P}(\alpha, A, \beta)$. 
Let $X^{\prime}=\bigcup\left\{X_{n}: n<\omega\right\}$ and let $K$ be the infinum of $\left\langle F_{n}: n<\omega\right\rangle$. For each $\xi$ in $\operatorname{dom}(K)$, write $K(\xi)$ as $\left\langle a_{i}^{\xi}: i \leq \gamma_{\xi}\right\rangle$. Then:

(1) $\bigcup X^{\prime}=N \cap \beta$,

(2) $\operatorname{dom}(K)=N \cap[\alpha, \beta) \cap \operatorname{cof}\left(\omega_{1}\right)$,

(3) for all $\xi$ in $\operatorname{dom}(K), \gamma_{\xi}=N \cap \omega_{1}$ and $a_{\gamma_{\xi}}^{\xi}=N \cap \xi$.

Proof. For each $n<\omega, X_{n}$ is in $N$. Since $X_{n}$ is countable, $X_{n} \subseteq N$. It follows that $X^{\prime}=\bigcup\left\{X_{n}: n<\omega\right\} \subseteq N$, so if $b$ is in $X^{\prime}$, then $b$ is in $N$, and therefore since $b$ is countable, $b \subseteq N \cap \beta$. It follows that $\bigcup X^{\prime} \subseteq N \cap \beta$. On the other hand, if $\nu$ is in $N \cap \beta$, then by Lemma 3.5 there is a dense set $D$ of conditions $(X, F)$ such that $\nu$ is in $\bigcup X$. By elementarity, $D$ is in $N$, so fix $n<\omega$ such that $\left(X_{n}, F_{n}\right)$ is in $D$. Then $\nu$ is in $\bigcup X_{n} \subseteq \bigcup X^{\prime}$, so $\nu$ is in $\bigcup X^{\prime}$. Thus $\bigcup X^{\prime}=N \cap \beta$. Statements (2) and $(3)$ can be proven by similar arguments, using Lemma 3.6.

The next result is our main tool for constructing lower bounds of descending sequences.

Proposition 3.8. Let $\theta$ be a regular cardinal much larger than $\mathbb{P}(\alpha, A, \beta)$. Let $N$ be a countable elementary substructure of $H(\theta)$ such that $\mathbb{P}(\alpha, A, \beta)$ is in $N$, and moreover, if $\operatorname{cf}(\alpha)=\omega_{1}$, then $N \cap \alpha$ is not in $A$. Let $\left\langle\left(X_{n}, F_{n}\right): n<\omega\right\rangle$ be an $N$-generic sequence for $\mathbb{P}(\alpha, A, \beta)$.

Let $X^{\prime}=\bigcup\left\{X_{n}: n<\omega\right\}$ and let $K$ be the infinum of $\left\langle F_{n}: n<\omega\right\rangle$. Suppose that $Y$ is a countable subset of $P_{\omega_{1}}(\beta)$ such that $X^{\prime} \subseteq Y$, and for all $b$ in $Y \backslash X^{\prime}$, either $b=N \cap \beta$ or there is an uncountable ordinal $\nu$ in $(\alpha, \beta) \cap N \cap \operatorname{cof}(\omega)$ such that $b=N \cap \nu$. Then $(Y, K)$ is a condition in $\mathbb{P}(\alpha, A, \beta)$ and $(Y, K) \leq\left(X_{n}, F_{n}\right)$ for all $n<\omega$.

Proof. We prove that $(Y, K)$ is a condition in $\mathbb{P}(\alpha, A, \beta)$. Note that properties (1), (3), and (4) of Definition 3.1 are obvious, and (2) follows from the fact that $\alpha$ is in $N$. To prove (5) and (6), consider $\xi$ in $\operatorname{dom}(K)$. Let $K(\xi)=\left\langle a_{i}^{\xi}: i \leq \gamma_{\xi}\right\rangle$. By Lemma 3.7, $U Y=N \cap \beta, \xi \in N$, and $a_{\gamma_{\xi}}^{\xi}=N \cap \xi$, so $a_{\gamma_{\xi}}^{\xi}=N \cap \xi \subseteq N \cap \beta=\bigcup Y$, proving (6).

To prove (5), let $i \leq \gamma_{\xi}$, and we show $a_{i}^{\xi}$ is not in $Y \cup A$. First suppose $i<\gamma_{\xi}$. Then $a_{i}^{\xi}$ is in $N$. Note that every set in $Y \backslash X^{\prime}$ is not in $N$, so if $a_{i}^{\xi}$ is in $Y$, then it is in $X^{\prime}$. But if $a_{i}^{\xi}$ is in $X^{\prime}$, then we can find $n<\omega$ such that $a_{i}^{\xi}$ is in $X_{n}$ and also appears on the sequence $F_{n}(\xi)$, contradicting the fact that $\left(X_{n}, F_{n}\right)$ is a condition. Therefore, $a_{i}^{\xi}$ is not in $Y$. For some $n<\omega, a_{i}^{\xi}$ is on the sequence $F_{n}(\xi)$, which implies by definition that $a_{i}^{\xi}$ is not in $A$. Therefore, $a_{i}^{\xi}$ is not in $Y \cup A$.

Now assume $i=\gamma_{\xi}$. Then by Lemma 3.7, $a_{\gamma_{\xi}}^{\xi}=N \cap \xi$. In particular, $a_{\gamma_{\xi}}^{\xi}$ is not in $N$, so $a_{\gamma_{\xi}}^{\xi}$ is not in $X^{\prime}$. If $\xi=\alpha$, then $\alpha$ has cofinality $\omega_{1}$. By the assumptions on $N, N \cap \xi=N \cap \alpha=a_{\gamma_{\xi}}^{\xi}$ is not in $A$. If $\xi>\alpha$, then $\alpha \in N \cap \xi=a_{\gamma_{\xi}}^{\xi}$, and therefore $a_{\gamma_{\xi}}^{\xi}$ is not in $A$. Therefore, we have proven that $a_{\gamma_{\xi}}^{\xi}$ is not in $X^{\prime} \cup A$.

Let $b$ be in $Y \backslash X^{\prime}$, and we show $b$ is not equal to $a_{\gamma_{\xi}}^{\xi}$. First suppose $b=N \cap \beta$. Then $\xi \in N \cap \beta=b$, whereas $a_{\gamma_{\xi}}^{\xi}$ is a subset of $\xi$, so $b$ is not equal to $a_{\gamma_{\xi}}^{\xi}$. Now suppose $b=N \cap \nu$ for some uncountable ordinal $\nu$ in $(\alpha, \beta) \cap N \cap \operatorname{cof}(\omega)$. Since $\nu$ has cofinality $\omega$ and $\xi$ has cofinality $\omega_{1}$, either $\xi<\nu$ or $\nu<\xi$. If $\xi<\nu$, then $\xi$ is in $N \cap \nu=b$, whereas $a_{\gamma_{\xi}}^{\xi} \subseteq \xi$, so $a_{\gamma_{\xi}}^{\xi}$ is not equal to $b$. If $\nu<\xi$, then $\nu$ is in $N \cap \xi=a_{\gamma_{\xi}}^{\xi}$, whereas $b \subseteq \nu$, so $a_{\gamma_{\xi}}^{\xi}$ is not equal to $b$. This completes the proof that $(Y, K)$ is in $\mathbb{P}(\alpha, A, \beta)$. 
Let $n<\omega$ be given, and we show $(Y, K) \leq\left(X_{n}, F_{n}\right)$. Properties (a), (c), and (d) of Definition 3.1 are immediate. Suppose $b$ is in $Y \backslash X_{n}$, and we show $b \nsubseteq \subseteq \cup X_{n}$. If $b$ is in $X^{\prime}$, then there is $m>n$ such that $b$ is in $X_{m} \backslash X_{n}$. Since $\left(X_{m}, F_{m}\right) \leq\left(X_{n}, F_{n}\right)$, $b \nsubseteq \bigcup X_{n}$. Now suppose $b$ is not in $X^{\prime}$. Then $b=N \cap \nu$, where either $\nu=\beta$ or $\nu$ is an uncountable ordinal in $(\alpha, \beta) \cap N \cap \operatorname{cof}(\omega)$. Suppose for a contradiction that $b \subseteq \bigcup X_{n}$. Then $b=b \cap \nu \subseteq\left(\bigcup X_{n}\right) \cap \nu \subseteq N \cap \nu=b$, so $\left(\bigcup X_{n}\right) \cap \nu=N \cap \nu$. But $\bigcup X_{n}$ and $\nu$ are in $N$, so by elementarity, $\left(\bigcup X_{n}\right) \cap \nu$ is in $N$. Therefore, $N \cap \nu$ is in $N$, which is impossible. Indeed $b \nsubseteq \bigcup X_{n}$.

Theorem 3.9. The forcing poset $\mathbb{P}(\alpha, A, \beta)$ is $\omega_{1}$-distributive.

Proof. Let $\left\{D_{n}: n<\omega\right\}$ be a collection of dense open subsets of $\mathbb{P}(\alpha, A, \beta)$. Let $(X, F)$ be a condition. We find a condition $(Y, K) \leq(X, F)$ in $\bigcap\left\{D_{n}: n<\omega\right\}$.

Fix a regular cardinal $\theta$ much larger than $\mathbb{P}(\alpha, A, \beta)$. Choose a countable elementary substructure $N$ of $H(\theta)$ which contains as members the objects $\mathbb{P}(\alpha, A, \beta)$, $(X, F)$, and $\left\{D_{n}: n<\omega\right\}$, and moreover, if $\operatorname{cf}(\alpha)=\omega_{1}$, then $N \cap \alpha$ is not in $A$. This is possible since if $\operatorname{cf}(\alpha)=\omega_{1}$, then $P_{\omega_{1}}(\alpha) \backslash A$ is stationary in $P_{\omega_{1}}(\alpha)$.

Choose an $N$-generic sequence $\left\langle\left(X_{n}, F_{n}\right): n<\omega\right\rangle$ with $\left(X_{0}, F_{0}\right)=(X, F)$. Let $Y=\bigcup\left\{X_{n}: n<\omega\right\}$ and let $K$ be the infinum of the sequence $\left\langle F_{n}: n<\omega\right\rangle$. By Proposition 3.8, $(Y, K)$ is a condition and $(Y, K) \leq\left(X_{n}, F_{n}\right)$ for all $n<\omega$. In particular, $(Y, K) \leq(X, F)$. Let $n<\omega$ be given. Then $D_{n}$ is in $N$; so for some $m,\left(X_{m}, F_{m}\right)$ is in $D_{n}$. Since $D_{n}$ is open, $(Y, K)$ is in $D_{n}$. Thus $(Y, K)$ is in $\bigcap\left\{D_{n}: n<\omega\right\}$.

Theorem 3.10. If $2^{\omega}=\omega_{1}$, then $\mathbb{P}(\alpha, A, \beta)$ is $\omega_{2}$-c.c.

Proof. We will prove that $\mathbb{P}(\alpha, A, \beta)$ is $\omega_{2}$-Knaster. Let $\left\langle\left(X_{j}, F_{j}\right): j<\omega_{2}\right\rangle$ be a sequence of conditions in $\mathbb{P}(\alpha, A, \beta)$. For each $j<\omega_{2}$ and $\xi \in \operatorname{dom}\left(F_{j}\right)$, let $F_{j}(\xi)=\left\langle a(j)_{i}^{\xi}: i \leq \gamma_{\xi}^{j}\right\rangle$. We will thin out this sequence of conditions in five steps.

First, apply the $\Delta$-System Lemma to the sequence of countable sets $\left\langle\operatorname{dom}\left(F_{j}\right)\right.$ : $\left.j<\omega_{2}\right\rangle$ to obtain an unbounded set $Z_{0} \subseteq \omega_{2}$ and a countable set $B$ such that for all $j<k$ in $Z_{0}, \operatorname{dom}\left(F_{j}\right) \cap \operatorname{dom}\left(F_{k}\right)=B$.

Second, an easy counting argument using $\mathrm{CH}$ shows there are at most $\omega_{1}$ many possibilities for a sequence of the form $\left\langle\gamma_{\xi}^{j}: \xi \in B\right\rangle$, for $j$ in $Z_{0}$. Therefore, we can fix an unbounded set $Z_{1} \subseteq Z_{0}$ and a sequence $\left\langle\gamma_{\xi}: \xi \in B\right\rangle$ such that for all $j \in Z_{1}$ and $\xi \in B, \gamma_{\xi}^{j}=\gamma_{\xi}$.

Third, another straightforward counting argument shows there are at most $\omega_{1}$ many possibilities for a sequence of the form $\left\langle a(j)_{i}^{\xi}: \xi \in B, i \leq \gamma_{\xi}\right\rangle$, for $j \in Z_{1}$. Fix an unbounded set $Z_{2} \subseteq Z_{1}$ and a sequence $\left\langle a_{i}^{\xi}: \xi \in B, i \leq \gamma_{\xi}\right\rangle$ such that for all $j \in Z_{2}, \xi \in B$, and $i \leq \gamma_{\xi}, a(j)_{i}^{\xi}=a_{i}^{\xi}$.

Fourth, apply the $\Delta$-System Lemma to the sequence of countable sets $\left\langle\bigcup X_{j}\right.$ : $\left.j \in Z_{2}\right\rangle$ to obtain an unbounded set $Z_{3} \subseteq Z_{2}$ and a countable set $E$ such that for all $j<k$ in $Z_{3},\left(\bigcup X_{j}\right) \cap\left(\bigcup X_{k}\right)=E$.

Fifth, a routine counting argument shows there are at most $\omega_{1}$ possibilities for a set of the form $P(E) \cap X_{j}$, for $j \in Z_{3}$. Therefore, we can choose an unbounded set $Z_{4} \subseteq Z_{3}$ so that for all $j<k$ in $Z_{4}, P(E) \cap X_{j}=P(E) \cap X_{k}$.

Claim 3.11. Let $j$ and $k$ be distinct ordinals in $Z_{4}$, and suppose $b$ is in $X_{j} \backslash X_{k}$. Then $b$ is not a subset of $\bigcup X_{k}$. 
Proof. Suppose for a contradiction that $b$ is in $X_{j} \backslash X_{k}$ and $b \subseteq \bigcup X_{k}$. Since $b$ is in $X_{j}, b \subseteq \bigcup X_{j}$. Therefore, $b \subseteq\left(\bigcup X_{j}\right) \cap\left(\bigcup X_{k}\right)=E$ and $b$ is in $P(E) \cap X_{j}$. However, $P(E) \cap X_{j}=P(E) \cap X_{k}$. Therefore, $b$ is in $X_{k}$, which is a contradiction.

Let $j<k$ be in $Z_{4}$, and we prove that $\left(X_{j}, F_{j}\right)$ and $\left(X_{k}, F_{k}\right)$ are compatible. Define $(Y, K)$ as follows. Let $Y=X_{j} \cup X_{k}$. Let $\operatorname{dom}(K)=\operatorname{dom}\left(F_{j}\right) \cup \operatorname{dom}\left(F_{k}\right)$. If $\xi$ is in $\operatorname{dom}\left(F_{j}\right) \backslash \operatorname{dom}\left(F_{k}\right)$, let $K(\xi)=F_{j}(\xi)$, and if $\xi$ is in $\operatorname{dom}\left(F_{k}\right) \backslash \operatorname{dom}\left(F_{j}\right)$, let $K(\xi)=F_{k}(\xi)$. If $\xi$ is $\operatorname{dom}\left(F_{j}\right) \cap \operatorname{dom}\left(F_{k}\right)=B$, then $F_{j}(\xi)$ and $F_{k}(\xi)$ are both equal to $\left\langle a_{i}^{\xi}: i \leq \gamma_{\xi}\right\rangle$, and we define $K(\xi)$ to be this sequence. This completes the definition of $(Y, K)$.

We prove that $(Y, K)$ is a condition. Properties (1), (2), (3), and (4) of Definition 3.1 are immediate. For $(5)$ and (6), suppose $\xi$ is in $\operatorname{dom}(K)$. Let $K(\xi)=\left\langle a_{i}^{\xi}: i \leq\right.$ $\left.\gamma_{\xi}\right\rangle$. Consider $i \leq \gamma_{\xi}$. Since $a_{i}^{\xi}$ appears on either the sequence $F_{j}(\xi)$ or $F_{k}(\xi), a_{i}^{\xi}$ is either a subset of $\bigcup X_{j}$ or of $\bigcup X_{k}$. These sets are in turn subsets of $\bigcup Y$, so $a_{i}^{\xi} \subseteq \bigcup Y$. This verifies (6).

It remains to show that $a_{i}^{\xi}$ is not in $Y \cup A$. Since $a_{i}^{\xi}$ appears on either $F_{j}(\xi)$ or $F_{k}(\xi)$, it is not in $A$. Now we show $a_{i}^{\xi}$ is not in $Y$. First assume $\xi$ is in $\operatorname{dom}\left(F_{j}\right) \backslash \operatorname{dom}\left(F_{k}\right)$. Then $K(\xi)=F_{j}(\xi)$, so $a_{i}^{\xi}$ appears on the sequence $F_{j}(\xi)$. Since $\left(X_{j}, F_{j}\right)$ is a condition, $a_{i}^{\xi}$ is not in $X_{j}$ and $a_{i}^{\xi} \subseteq \bigcup X_{j}$. If for a contradiction $a_{i}^{\xi}$ is in $Y$, then $a_{i}^{\xi}$ is in $X_{k} \backslash X_{j}$. By Claim 3.11, $a_{i}^{\xi}$ is not a subset of $\bigcup X_{j}$, which is a contradiction. The case when $\xi$ is in $\operatorname{dom}\left(F_{k}\right) \backslash \operatorname{dom}\left(F_{j}\right)$ is the same, with the roles of $j$ and $k$ reversed. Now assume $\xi$ is in $\operatorname{dom}\left(F_{j}\right) \cap \operatorname{dom}\left(F_{k}\right)=B$. Then $a_{i}^{\xi}$ appears on the sequence $F_{j}(\xi)=F_{k}(\xi)$. Since $\left(X_{j}, F_{j}\right)$ and $\left(X_{k}, F_{k}\right)$ are conditions, $a_{i}^{\xi}$ is neither in $X_{j}$ nor in $X_{k}$. Therefore $a_{i}^{\xi}$ is not in $Y=X_{j} \cup X_{k}$. This completes the proof that $(Y, K)$ is in $\mathbb{P}(\alpha, A, \beta)$.

For proving that $(Y, K) \leq\left(X_{j}, F_{j}\right),\left(X_{k}, F_{k}\right)$, properties (a), (c), and (d) of Definition 3.1 are immediate, and (b) follows from Claim 3.11.

\section{The SET $S(\alpha, A, \beta)$}

Fix for the remainder of the section $\alpha, \beta$, and $A$ as described in the assumptions of Definition 3.1. Specifically, $\alpha<\beta \leq \omega_{2}, \beta$ has uncountable cofinality, $A$ is a subset of $P_{\omega_{1}}(\alpha)$, and if $\operatorname{cf}(\alpha)=\omega_{1}$, then $P_{\omega_{1}}(\alpha) \backslash A$ is stationary in $P_{\omega_{1}}(\alpha)$. Also assume $2^{\omega}=\omega_{1}$.

Notation 4.1. Let $\dot{S}(\alpha, A, \beta)$ be a $\mathbb{P}(\alpha, A, \beta)$-name such that

$$
\Vdash \dot{S}(\alpha, A, \beta)=\bigcup\{X: \exists F(X, F) \in \dot{H}\},
$$

where $\dot{H}$ is the canonical name for the generic filter.

Notation 4.2. In the case $\alpha=0$ and $A=\emptyset$, let $\dot{S}_{\beta}$ denote $\dot{S}(0, \emptyset, \beta)$.

Consider a generic filter $H$ on $\mathbb{P}(\alpha, A, \beta)$ over $V$. Since $\mathbb{P}(\alpha, A, \beta)$ is $\omega_{1}$-distributive and $\omega_{2}$-c.c., all cardinals and cofinalities are preserved in $V[H]$. Also by distributivity, $P_{\omega_{1}}(\beta)$ is computed the same in $V$ and in $V[H]$. The set $S(\alpha, A, \beta)$ is a subset of $P_{\omega_{1}}(\beta)$ in $V[H]$. Let $\xi$ be in $[\alpha, \beta) \cap \operatorname{cof}\left(\omega_{1}\right)$. By Lemma 3.6, if we take the union of all sequences of the form $F(\xi)$, where $(X, F) \in H$ for some $X$, and $\xi \in \operatorname{dom}(F)$, then we obtain an increasing and continuous sequence $\left\langle a_{i}^{\xi}: i<\omega_{1}\right\rangle$ cofinal in $P_{\omega_{1}}(\xi)$. 
Lemma 4.3. In $V[H]$, for all $\xi$ in $[\alpha, \beta) \cap \operatorname{cof}\left(\omega_{1}\right)$, the set $\left\{a_{i}^{\xi}: i<\omega_{1}\right\}$ is a club subset of $P_{\omega_{1}}(\xi)$ which is disjoint from $S(\alpha, A, \beta)$. Therefore, $S(\alpha, A, \beta)$ does not reflect to any ordinal in $[\alpha, \beta)$ with cofinality $\omega_{1}$.

Proof. If $b$ is in $S(\alpha, A, \beta)$, fix $(X, F)$ in $H$ such that $b \in X$. Suppose for a contradiction that $b$ is equal to $a_{i}^{\xi}$ for some $\xi$ in $[\alpha, \beta) \cap \operatorname{cof}\left(\omega_{1}\right)$ and $i<\omega_{1}$. Then there is $(Y, K) \leq(X, F)$ such that $b$ appears on the sequence $F(\xi)$. But then $b$ is in $Y$ and on the sequence $F(\xi)$, which contradicts property (5) of Definition 3.1.

Note that the statement that $S(\alpha, A, \beta)$ does not reflect to any ordinal in $[\alpha, \beta) \cap$ $\operatorname{cof}\left(\omega_{1}\right)$ is upwards absolute between $V[H]$ and any outer model $W$ of $V[H]$ with the same $\omega_{1}$.

Proposition 4.4. The forcing poset $\mathbb{P}(\alpha, A, \beta)$ forces that $\dot{S}(\alpha, A, \beta)$ is a stationary subset of $P_{\omega_{1}}(\beta)$.

Proof. Let $(X, F)$ be a condition in $\mathbb{P}(\alpha, A, \beta)$ and suppose $(X, F)$ forces $\dot{C}$ is a club subset of $P_{\omega_{1}}(\beta)$. We will find $(Y, K) \leq(X, F)$ and a set $z$ such that $(Y, K) \Vdash$ $z \in \dot{S}(\alpha, A, \beta) \cap \dot{C}$.

Fix a regular cardinal $\theta$ much larger than $\mathbb{P}(\alpha, A, \beta)$ and $\dot{C}$. Let $N$ be a countable elementary substructure of $H(\theta)$ which contains as members the objects $\mathbb{P}(\alpha, A, \beta)$, $(X, F)$, and $\dot{C}$, and moreover, if $\operatorname{cf}(\alpha)=\omega_{1}$, then $N \cap \alpha$ is in $P_{\omega_{1}}(\alpha) \backslash A$. Let $\left\langle\left(X_{n}, F_{n}\right): n<\omega\right\rangle$ be an $N$-generic sequence with $\left(X_{0}, F_{0}\right)=(X, F)$.

Let $X^{\prime}=\bigcup\left\{X_{n}: n<\omega\right\}$. Define $Y=X^{\prime} \cup\{N \cap \beta\}$. Let $K$ be the infinum of the sequence $\left\langle F_{n}: n<\omega\right\rangle$. By Proposition 3.8, $(Y, K)$ is a condition in $\mathbb{P}(\alpha, A, \beta)$, and $(Y, K) \leq\left(X_{n}, F_{n}\right)$ for all $n<\omega$. In particular, $(Y, K) \leq(X, F)$ and $(Y, K)$ is an $N$-generic condition. Since $N \cap \beta \in Y,(Y, K) \Vdash N \cap \beta \in \dot{S}(\alpha, A, \beta)$.

Since $\dot{C}$ is in $N, \mathbb{P}(\alpha, A, \beta)$ forces that $N[\dot{H}] \cap \beta \in \dot{C}$, where $\dot{H}$ is the canonical $\mathbb{P}(\alpha, A, \beta)$-name for the generic filter. As $(Y, K)$ is $N$-generic, $(Y, K) \Vdash N[\dot{H}] \cap \beta=$ $N \cap \beta$, so $(Y, K) \Vdash N \cap \beta \in \dot{S}(\alpha, A, \beta) \cap \dot{C}$.

The next easy lemma will be used frequently throughout the paper.

Lemma 4.5. Let $(X, F)$ be a condition in $\mathbb{P}(\alpha, A, \beta)$. Then

$$
(X, F) \Vdash \dot{S}(\alpha, A, \beta) \cap P(\bigcup X)=X .
$$

Proof. Clearly $(X, F) \Vdash X \subseteq \dot{S}(\alpha, A, \beta) \cap P(\bigcup X)$. Let $H$ be a generic filter on $\mathbb{P}(\alpha, A, \beta)$ with $(X, F)$ in $H$. Suppose for a contradiction that there is $x$ in $S(\alpha, A, \beta) \cap P(\bigcup X)$ which is not in $X$. Fix a condition $(Y, K)$ in $H$ such that $x$ is in $Y$. Choose $(Z, L)$ in $H$ which is below $(X, F)$ and $(Y, K)$. Then $x$ is in $Z \backslash X$ and $x \subseteq \bigcup X$, which contradicts the fact that $(Z, L) \leq(X, F)$.

Lemma 4.6. The forcing poset $\mathbb{P}(\alpha, A, \beta)$ forces that for any countable set $B \subseteq \beta$,

$$
|\dot{S}(\alpha, A, \beta) \cap P(B)| \leq \aleph_{0} .
$$

Proof. If $B$ is a countable subset of $\beta$ in a generic extension by $\mathbb{P}(\alpha, A, \beta)$, then since $\mathbb{P}(\alpha, A, \beta)$ is $\omega_{1}$-distributive, $B$ is in the ground model. There are densely many conditions of the form $(X, F)$, where $B \subseteq \bigcup X$. If $(X, F)$ is such a condition, then by Lemma $4.5,(X, F)$ forces that any subset of $B$ which is in $\dot{S}(\alpha, A, \beta)$ is in $\dot{S}(\alpha, A, \beta) \cap P(\bigcup X)=X$. Therefore, $(X, F) \Vdash \dot{S}(\alpha, A, \beta) \cap P(B) \subseteq X$. Since $X$ is countable, $(X, F) \Vdash|\dot{S}(\alpha, A, \beta) \cap P(B)| \leq \aleph_{0}$. 
The next technical, unmotivated lemma will be crucial to several arguments in later sections.

Lemma 4.7. Let $\theta \geq \omega_{2}$ be a regular cardinal. Then:

(1) $\mathbb{P}(\alpha, A, \beta)$ forces that there are stationarily many $N$ in $P_{\omega_{1}}(H(\theta))$ such that $\dot{S}(\alpha, A, \beta) \cap P(N \cap \beta) \subseteq N$.

(2) Assume $\operatorname{cf}(\alpha)=\omega_{1}$, and let $T$ be a stationary subset of $P_{\omega_{1}}(\alpha)$ which is disjoint from $A$. Then $\mathbb{P}(\alpha, A, \beta)$ forces that there are stationarily many $N$ in $P_{\omega_{1}}(H(\theta))$ such that $\dot{S}(\alpha, A, \beta) \cap P(N \cap \beta) \subseteq N$ and $N \cap \alpha \in T$.

Proof. Let $(X, F)$ be a condition in $\mathbb{P}(\alpha, A, \beta)$, and suppose $(X, F)$ forces $\dot{C}$ is a club subset of $P_{\omega_{1}}(H(\theta)$ ). (By $H(\theta)$, we mean $H(\theta)$ as computed in the generic extension.) Let $\chi$ be a regular cardinal much larger than $\mathbb{P}(\alpha, A, \beta), \theta$, and $\dot{C}$. Let $M$ be a countable elementary substructure of $H(\chi)$ which contains as members the objects $\mathbb{P}(\alpha, A, \beta),(X, F), \theta$, and $\dot{C}$, and if $\operatorname{cf}(\alpha)=\omega_{1}$, then $M \cap \alpha \in P_{\omega_{1}}(\alpha) \backslash A$. In addition, in case (2), also assume $M \cap \alpha$ is in $T$.

Let $\left\langle\left(X_{n}, F_{n}\right): n<\omega\right\rangle$ be an $M$-generic sequence with $\left(X_{0}, F_{0}\right)=(X, F)$. Let $Y=\bigcup\left\{X_{n}: n<\omega\right\}$ and let $K$ be the infinum of the sequence $\left\langle F_{n}: n<\omega\right\rangle$. By Proposition 3.8, $(Y, K)$ is in $\mathbb{P}(\alpha, A, \beta)$, and $(Y, K) \leq\left(X_{n}, F_{n}\right)$ for all $n<\omega$. In particular, $(Y, K) \leq(X, F)$ and $(Y, K)$ is $M$-generic. Since $\bigcup Y=M \cap \beta$, Lemma 4.5 implies $(Y, K) \Vdash \dot{S}(\alpha, A, \beta) \cap P(M \cap \beta)=Y$.

Let $H$ be a generic filter on $\mathbb{P}(\alpha, A, \beta)$ with $(Y, K) \in H$. Since $(Y, K)$ is $M$ generic, $M[H] \cap V=M$. Also $S(\alpha, A, \beta) \cap P(M \cap \beta)=Y$. Let $N=M[H] \cap$ $H(\theta)^{V[H]}$. We show that $N$ is as desired.

Since $\theta$ is in $M, H(\theta)^{V[H]}$ is in $M[H]$, so by standard arguments, $N$ is an elementary substructure of $H(\theta)^{V[H]}$. As $\dot{C}$ is in $M, N$ is in $C$. Also, since $(Y, K)$ is $M$-generic, $N \cap \beta=M[H] \cap \beta=M \cap \beta$ and $N \cap \alpha=M[H] \cap \alpha=M \cap \alpha$. If $\operatorname{cf}(\alpha)=\omega_{1}$, then $N \cap \alpha=M \cap \alpha$ is in $T$.

It suffices to show $S(\alpha, A, \beta) \cap P(N \cap \beta) \subseteq N$. Let $b$ be in $S(\alpha, A, \beta) \cap P(N \cap \beta)$. Since $N \cap \beta=M \cap \beta, b$ is in $S(\alpha, A, \beta) \cap P(M \cap \beta)=Y$, so $b$ is in $Y$. However, $Y=\bigcup\left\{X_{n}: n<\omega\right\}$, so fix $n<\omega$ such that $b$ is in $X_{n}$. Then since $X_{n} \in M$ and $X_{n}$ is countable, $X_{n} \subseteq M$, so $b$ is in $M$. Hence $b$ is in $M[H]$. Since $\beta \leq \theta, b$ is in $M[H] \cap H(\theta)=N$.

Corollary 4.8. Assume that $\operatorname{cf}(\alpha)=\omega_{1}$, and let $T$ be a stationary subset of $P_{\omega_{1}}(\alpha)$ which is disjoint from $A$. Then $\mathbb{P}(\alpha, A, \beta)$ forces that $T$ is stationary in $P_{\omega_{1}}(\alpha)$.

Proof. Let $H$ be a generic filter on $\mathbb{P}(\alpha, A, \beta)$ over $V$. In $V[H]$ let $C$ be a club subset of $P_{\omega_{1}}(\alpha)$. Fix a regular cardinal $\theta$ in $V[H]$ much larger than $\omega_{2}$ and $C$. Note that in $V, \theta \geq \omega_{2}$ is regular. By Lemma 4.7, we can find a countable elementary substructure $M$ of $H(\theta)$ with $C$ in $M$ such that $M \cap \alpha \in T$. Since $C$ is in $M$, $M \cap \alpha \in C \cap T$.

Corollary 4.9. The forcing poset $\mathbb{P}(\alpha, A, \beta)$ forces that $P_{\omega_{1}}(\beta) \backslash \dot{S}(\alpha, A, \beta)$ is stationary in $P_{\omega_{1}}(\beta)$.

Proof. Let $H$ be a generic filter on $\mathbb{P}(\alpha, A, \beta)$ over $V$. In $V[H]$ consider a function $F:[\beta]^{<\omega} \rightarrow \beta$. Fix a regular cardinal $\theta$ much larger than $\beta$. By Lemma 4.7 , we can choose a countable elementary substructure $M$ of $H(\theta)$ with $F$ in $M$ such that $S(\alpha, A, \beta) \cap P(M \cap \beta) \subseteq M$. By elementarity, $M \cap \beta$ is closed under $F$. Since $M \cap \beta$ 
is in $P(M \cap \beta)$ but not in $M, M \cap \beta$ is not in $S(\alpha, A, \beta)$. Thus $P_{\omega_{1}}(\beta) \backslash S(\alpha, A, \beta)$ is stationary in $P_{\omega_{1}}(\beta)$.

We end this section by proving a general lemma regarding subsets of $P_{\omega_{1}}(\beta)$ which do not reflect to any ordinal of cofinality $\omega_{1}$. We will use this result in Section 12 .

Lemma 4.10. Let $\beta \leq \omega_{2}$ be an ordinal with uncountable cofinality. Suppose $S$ is a subset of $P_{\omega_{1}}(\beta)$ which satisfies the property that for all $\nu$ in $\beta \cap \operatorname{cof}\left(\omega_{1}\right), S$ does not reflect to $\nu$. Then there is a club set $C \subseteq P_{\omega_{1}}(\beta)$ such that for all $b$ in $C$, for all $\nu$ in $b \cap \operatorname{cof}\left(\omega_{1}\right), b \cap \nu$ is not in $S$.

Proof. Suppose for a contradiction there is a stationary set $U \subseteq P_{\omega_{1}}(\beta)$ such that for all $b$ in $U$, there is an ordinal $\nu$ in $b \cap \operatorname{cof}\left(\omega_{1}\right)$ such that $b \cap \nu$ is in $S$. By Fodor's Lemma, fix a stationary set $U^{\prime} \subseteq U$ and an ordinal $\nu$ in $\beta \cap \operatorname{cof}\left(\omega_{1}\right)$ such that for all $b$ in $U^{\prime}, \nu$ is in $b$ and $b \cap \nu$ is in $S$.

We prove that $S \cap P_{\omega_{1}}(\nu)$ is stationary in $P_{\omega_{1}}(\nu)$, which contradicts our assumption on $S$. Therefore, consider a function $F:[\nu]^{<\omega} \rightarrow \nu$. Since $U^{\prime}$ is stationary, fix $b$ in $U^{\prime}$ such that $b$ is closed under $F$. Then $b \cap \nu$ is closed under $F$. Since $b$ is in $U^{\prime}, b \cap \nu$ is in $S$.

\section{FACTORIZATION}

For the remainder of the section fix ordinals $\nu<\beta \leq \omega_{2}$ with uncountable cofinality. Assume $2^{\omega}=\omega_{1}$. Recall that $\mathbb{P}_{\nu}$ and $\dot{S}_{\nu}$ abbreviate $\mathbb{P}(0, \emptyset, \nu)$ and $\dot{S}(0, \emptyset, \nu)$. In this section we prove that $\mathbb{P}_{\beta}$ factors as the two-step iteration $\mathbb{P}_{\nu} *$ $\mathbb{P}\left(\nu, \dot{S}_{\nu}, \beta\right)$. This factorization will be important for the purpose of extending an elementary embedding.

Notation 5.1. Let $(Y, K)$ be a condition in $\mathbb{P}_{\beta}$. For each $\xi$ in $\operatorname{dom}(K)$, let $K(\xi)=$ $\left\langle a_{i}^{\xi}: i \leq \gamma_{\xi}\right\rangle$. Define:

(1) $Y_{\nu}=\{b \in Y: b \subseteq \nu\}$,

(2) $K_{\nu}=K\lceil\nu$

(3) $Y^{\nu}=\{b \in Y: b \nsubseteq \nu\}$,

(4) $K^{\nu}=K \uparrow[\nu, \beta)$.

We define a dense suborder $\mathbb{P}_{\nu, \beta}$ of $\mathbb{P}_{\beta}$. The definition is designed so that if $(Y, K)$ is in $\mathbb{P}_{\nu, \beta}$, then $\left(Y_{\nu}, K_{\nu}\right)$ is in $\mathbb{P}_{\nu}$ and $\left(Y_{\nu}, K_{\nu}\right) \Vdash_{\mathbb{P}_{\nu}}\left(Y^{\nu}, K^{\nu}\right) \in \mathbb{P}\left(\nu, \dot{S}_{\nu}, \beta\right)$.

Definition 5.2. Let $\mathbb{P}_{\nu, \beta}$ be the suborder of $\mathbb{P}_{\beta}$ consisting of conditions $(Y, K)$ such that $\bigcup Y_{\nu}=\left(\bigcup Y^{\nu}\right) \cap \nu$.

Lemma 5.3. The suborder $\mathbb{P}_{\nu, \beta}$ is dense in $\mathbb{P}_{\beta}$.

Proof. Let $(X, F)$ be a condition in $\mathbb{P}_{\beta}$. Since $\bigcup X$ is countable and $\nu$ is uncountable, we can choose a countable set $e$ such that $(\bigcup X) \cap \nu \varsubsetneqq e \subseteq \nu$. Now choose $f$, a countable subset of $\beta$, such that $f \cap \nu=e, \nu \in f, \bigcup X \subseteq f$, and $f \backslash \nu \nsubseteq \bigcup X$.

Let $Y=X \cup\{e, f\}$. By two applications of Lemma 3.4, $(Y, F)$ is a condition in $\mathbb{P}_{\beta}$ which is below $(X, F)$. We show that $(Y, F)$ is in $\mathbb{P}_{\nu, \beta}$.

The set $e$ is in $Y_{\nu}$, and $e$ is the maximum element of $Y_{\nu}$. Therefore, $\bigcup Y_{\nu}=e$. Also, the set $f$ is the maximum element of $Y$, and since $f$ is in $Y^{\nu}, f$ is the maximum element of $Y^{\nu}$. It follows that $\bigcup Y^{\nu}=f$, so $\bigcup Y_{\nu}=e=f \cap \nu=\left(\bigcup Y^{\nu}\right) \cap \nu$. 
Lemma 5.4. Suppose $(Y, K)$ is in $\mathbb{P}_{\nu, \beta}$. For each $\xi$ in $\operatorname{dom}(K)$, let $K(\xi)=\left\langle a_{i}^{\xi}\right.$ : $\left.i \leq \gamma_{\xi}\right\rangle$. Then:

(1) $\bigcup Y=\bigcup Y^{\nu}$

(2) $\bigcup Y_{\nu}=(\bigcup Y) \cap \nu$,

(3) for all $\xi$ in $\operatorname{dom}(K), a_{\gamma_{\xi}}^{\xi} \subseteq \bigcup Y^{\nu}$,

(4) for all $\xi$ in $\operatorname{dom}(K)$ and $i \leq \gamma_{\xi}$, if $a_{i}^{\xi} \subseteq \nu$, then $a_{i}^{\xi} \subseteq \bigcup Y_{\nu}$.

Proof. (1) Clearly $\bigcup Y^{\nu} \subseteq \bigcup Y$. Suppose $\alpha$ is in $b$, where $b$ is in $Y$. If $b$ is in $Y^{\nu}$, then $\alpha$ is in $\bigcup Y^{\nu}$. Otherwise $b$ is in $Y_{\nu}$, so $\alpha$ is in $\bigcup Y_{\nu}$, which is equal to $\left(\bigcup Y^{\nu}\right) \cap \nu$. Therefore, $\alpha$ is in $\bigcup Y^{\nu}$. (2) $\bigcup Y_{\nu}=\left(\bigcup Y^{\nu}\right) \cap \nu$ by definition of $\mathbb{P}_{\nu, \beta}$, which is equal to $(\bigcup Y) \cap \nu$ by (1). (3) By Definition 3.1, $a_{\gamma_{\xi}}^{\xi} \subseteq \bigcup Y$, and $\bigcup Y=\bigcup Y^{\nu}$ by (1). (4) If $a_{i}^{\xi} \subseteq \nu$, then by $(3), a_{i}^{\xi} \subseteq\left(\bigcup Y^{\nu}\right) \cap \nu=\bigcup Y_{\nu}$.

Proposition 5.5. (I) If $(Y, K)$ is in $\mathbb{P}_{\nu, \beta}$, then $\left(Y_{\nu}, K_{\nu}\right)$ is in $\mathbb{P}_{\nu}$.

(II) Suppose $(Z, L) \leq(Y, K)$ in $\mathbb{P}_{\beta}$. If $(Z, L)$ and $(Y, K)$ are both in $\mathbb{P}_{\nu, \beta}$, then $\left(Z_{\nu}, L_{\nu}\right) \leq\left(Y_{\nu}, K_{\nu}\right)$ in $\mathbb{P}_{\nu}$.

(III) If $(Y, K)$ is in $\mathbb{P}_{\nu, \beta}$, then $\left(Y_{\nu}, K_{\nu}\right) \Vdash_{\mathbb{P}_{\nu}}\left(Y^{\nu}, K^{\nu}\right) \in \mathbb{P}\left(\nu, \dot{S}_{\nu}, \beta\right)$.

(IV) Suppose $(Z, L) \leq(Y, K)$ in $\mathbb{P}_{\beta}$. If $(Z, L)$ and $(Y, K)$ are both in $\mathbb{P}_{\nu, \beta}$, then $\left(Z_{\nu}, L_{\nu}\right) \Vdash_{\mathbb{P}_{\nu}}\left(Z^{\nu}, L^{\nu}\right) \leq\left(Y^{\nu}, K^{\nu}\right)$ in $\mathbb{P}\left(\nu, \dot{S}_{\nu}, \beta\right)$.

Proof. (I) We verify the properties of Definition 3.1 in the case of $\mathbb{P}_{\nu}=\mathbb{P}(0, \emptyset, \nu)$. Properties (1), (2), (3), and (4) are immediate. To show (5) and (6), consider $\xi$ in $\operatorname{dom}\left(K_{\nu}\right)=\operatorname{dom}(K) \cap \nu$. Let $K_{\nu}(\xi)=K(\xi)=\left\langle a_{i}^{\xi}: i \leq \gamma_{\xi}\right\rangle$ and let $i \leq \gamma_{\xi}$. Since $(Y, K)$ is in $\mathbb{P}_{\beta}, a_{i}^{\xi}$ is not in $Y$. However, $Y_{\nu} \subseteq Y$, so $a_{i}^{\xi}$ is not in $Y_{\nu}$. Also, $a_{i}^{\xi}$ is obviously not in $\emptyset$. This proves (5). For (6), we know that $a_{\gamma_{\xi}}^{\xi} \subseteq \cup Y$ and $a_{\gamma_{\xi}}^{\xi} \subseteq \xi \subseteq \nu$, so by Lemma 5.4(2), $a_{\gamma_{\xi}}^{\xi} \subseteq(\bigcup Y) \cap \nu=\bigcup Y_{\nu}$.

(II) Properties (a), (c), and (d) of Definition 3.1 are immediate. For (b), suppose $b$ is in $Z_{\nu} \backslash Y_{\nu}$. Then $b$ is in $Z$ and $b \subseteq \nu$. Since $b$ is not in $Y_{\nu}, b$ is not in $Y$, so $b$ is in $Z \backslash Y$. As $(Z, L) \leq(Y, K), b \nsubseteq \bigcup Y$, but $\bigcup Y_{\nu} \subseteq \bigcup Y$, so $b \nsubseteq \bigcup Y_{\nu}$.

(III) It is immediate that $\mathbb{P}_{\nu}$ forces that $\left(Y^{\nu}, K^{\nu}\right)$ satisfies properties (1), (2), (3), and (4) of Definition 3.1. For (5) and (6), consider $\xi$ in $\operatorname{dom}\left(K^{\nu}\right)=\operatorname{dom}(K) \cap[\nu, \beta)$. Let $K^{\nu}(\xi)=K(\xi)=\left\langle a_{i}^{\xi}: i \leq \gamma_{\xi}\right\rangle$. Let $i \leq \gamma_{\xi}$. Since $(Y, K)$ is in $\mathbb{P}_{\beta}, a_{i}^{\xi}$ is not in $Y$, so $a_{i}^{\xi}$ is not in $Y^{\nu}$. We need to show $\left(Y_{\nu}, K_{\nu}\right) \Vdash_{\mathbb{P}_{\nu}} a_{i}^{\xi} \notin \dot{S}_{\nu}$. If $a_{i}^{\xi}$ is not a subset of $\nu$, then this is clear. Suppose $a_{i}^{\xi} \subseteq \nu$. Then by Lemma 5.4(4), $a_{i}^{\xi} \subseteq \bigcup Y_{\nu}$. By Lemma 4.5, $\left(Y_{\nu}, K_{\nu}\right) \Vdash_{\mathbb{P}_{\nu}} \dot{S}_{\nu} \cap P\left(\bigcup Y_{\nu}\right)=Y_{\nu}$. However, $a_{i}^{\xi}$ is not in $Y$, and hence is not in $Y_{\nu}$, and $a_{i}^{\xi}$ is in $P\left(\bigcup Y_{\nu}\right)$. Therefore, $\left(Y_{\nu}, K_{\nu}\right) \Vdash a_{i}^{\xi} \notin \dot{S}_{\nu}$. This proves (5). By Lemma 5.4(3), $a_{\gamma_{\xi}}^{\xi} \subseteq \bigcup Y^{\nu}$, proving (6).

(IV) Since properties (a), (b), (c), and (d) of Definition 3.1 are absolute between $V$ and a generic extension by $\mathbb{P}_{\nu}$, it suffices to show that these properties hold in $V$. Properties (a), (c), and (d) are immediate. For (b), suppose $b$ is in $Z^{\nu} \backslash Y^{\nu}$. Then $b$ is $Z$ and $b \not \subset \nu$, but $b$ is not in $Y$, since otherwise $b$ would be in $Y^{\nu}$. Therefore, $b$ is in $Z \backslash Y$. Since $(Z, L) \leq(Y, K), b \nsubseteq \bigcup Y$. As $\bigcup Y^{\nu} \subseteq \bigcup Y, b \nsubseteq \bigcup Y^{\nu}$.

If $(Y, K)$ is in $\mathbb{P}_{\nu, \beta}$, then $\left(Y_{\nu}, K_{\nu}\right)$ is in $\mathbb{P}_{\nu}$. By Lemma $3.3, \mathbb{P}_{\nu} \subseteq \mathbb{P}_{\beta}$, so $\left(Y_{\nu}, K_{\nu}\right)$ is in $\mathbb{P}_{\beta}$ as well.

Lemma 5.6. Let $(Y, K)$ be in $\mathbb{P}_{\nu, \beta}$. Then $(Y, K) \leq\left(Y_{\nu}, K_{\nu}\right)$ in $\mathbb{P}_{\beta}$.

Proof. Properties (a), (c), and (d) of Definition 3.1 are immediate. For (b), if $b$ is in $Y \backslash Y_{\nu}$, then $b \nsubseteq \nu$. However, $\bigcup Y_{\nu} \subseteq \nu$, so $b \nsubseteq \bigcup Y_{\nu}$. 
Lemma 5.7. Let $(X, F)$ be a condition in $\mathbb{P}_{\nu}$. Suppose that $Y, K$ are sets such that $(X, F) \Vdash_{\mathbb{P}_{\nu}}(\check{Y}, \check{K}) \in \mathbb{P}\left(\nu, \dot{S}_{\nu}, \beta\right)$. Let $Z=X \cup Y$ and let $L=F \cup K$. Then $(Z, L)$ is in $\mathbb{P}_{\beta}$. Moreover, $Z_{\nu}=X, L_{\nu}=F, Z^{\nu}=Y$, and $L^{\nu}=K$.

Proof. Properties (1), (2), (3), and (4) of Definition 3.1 are immediate. For (5) and (6), let $\xi$ be in $\operatorname{dom}(L)$. Let $L(\xi)=\left\langle a_{i}^{\xi}: i \leq \gamma_{\xi}\right\rangle$. If $\xi$ is in $\operatorname{dom}(F)$, then $a_{\gamma_{\xi}}^{\xi} \subseteq \bigcup X \subseteq \bigcup Z$, and if $\xi$ is in $\operatorname{dom}(K)$, then $a_{\gamma_{\xi}}^{\xi} \subseteq \bigcup Y \subseteq \bigcup Z$. This proves (6).

To show (5), let $i \leq \gamma_{\xi}$, and we show $a_{i}^{\xi}$ is not in $Z \cup \emptyset=Z=X \cup Y$. First assume $\xi$ is in $\operatorname{dom}(K)$. Since $(X, F) \Vdash_{\mathbb{P}_{\nu}}(\check{Y}, \check{K}) \in \mathbb{P}\left(\nu, \dot{S}_{\nu}, \beta\right), a_{i}^{\xi}$ is not in $Y$. By Definition 3.1(5), $(X, F) \Vdash_{\mathbb{P}_{\nu}} a_{i}^{\xi} \notin \dot{S}_{\nu}$, which implies $a_{i}^{\xi} \notin X$. Therefore, $a_{i}^{\xi}$ is not in $Z$. Now suppose $\xi$ is in $\operatorname{dom}(F)$. Then clearly $a_{i}^{\xi}$ is not in $X$. Since $\operatorname{dom}(F) \subseteq \nu$, $a_{i}^{\xi}$ is a subset of $\nu$. However, for all $b$ in $Y, b \nsubseteq \nu$, so $a_{i}^{\xi}$ is not in $Y$.

Indeed, $(Z, L)$ is a condition in $\mathbb{P}_{\beta}$. The last statement of the lemma is immediate.

Lemma 5.8. There is a dense set of conditions in $\mathbb{P}_{\nu} * \mathbb{P}\left(\nu, \dot{S}_{\nu}, \beta\right)$ of the form $(X, F) *(\check{Y}, \check{K})$ such that $\bigcup X=(\bigcup Y) \cap \nu$. Suppose $(X, F) *(\check{Y}, \check{K})$ is such a condition. Let $Z=X \cup Y$ and $L=F \cup K$. Then $(Z, L)$ is in $\mathbb{P}_{\nu, \beta}$, and $\left(Z_{\nu}, L_{\nu}\right) *$ $\left(\check{Z}^{\nu}, \check{L}^{\nu}\right)$ is equal to $(X, F) *(\check{Y}, \check{K})$.

Proof. Let $\left(X_{0}, F_{0}\right) *\left(\dot{Y}_{0}, \dot{K}_{0}\right)$ be in $\mathbb{P}_{\nu} * \mathbb{P}\left(\nu, \dot{S}_{\nu}, \beta\right)$. Since $\mathbb{P}_{\nu}$ is $\omega_{1}$-distributive, we can fix $\left(X_{1}, F_{1}\right) \leq\left(X_{0}, F_{0}\right)$ and sets $Y_{1}$ and $K_{1}$ such that $\left(X_{1}, F_{1}\right)$ forces $\dot{Y}_{0}=\check{Y}_{1}$ and $\dot{K}_{0}=\check{K}_{1}$.

Choose a countable set $e$ such that $\bigcup X_{1} \varsubsetneqq e \subseteq \nu$ and $\left(\bigcup Y_{1}\right) \cap \nu \subseteq e$. Let $X_{2}=$ $X_{1} \cup\{e\}$. By Lemma 3.4, $\left(X_{2}, F_{1}\right)$ is in $\mathbb{P}_{\nu}$ and $\left(X_{2}, F_{1}\right) \leq\left(X_{1}, F_{1}\right)$. Now choose a countable set $f \subseteq \beta$ such that $f \cap \nu=e$ and $\left(\cup Y_{1}\right) \cup\{\nu\} \subsetneq f$. Let $Y_{2}=Y_{1} \cup\{f\}$. By Lemma 3.4, $\left(X_{2}, F_{1}\right)$ forces $\left(\check{Y}_{2}, \check{K}_{1}\right)$ is in $\mathbb{P}\left(\nu, \dot{S}_{\nu}, \beta\right)$ and $\left(\check{Y}_{2}, \check{K}_{1}\right) \leq\left(\check{Y}_{1}, \check{K}_{1}\right)$.

It follows that $\left(X_{2}, F_{1}\right) *\left(\check{Y}_{2}, \check{K}_{1}\right) \leq\left(X_{0}, F_{0}\right) *\left(\dot{Y}_{0}, \dot{K}_{0}\right)$. Note that $e$ is the maximum element of $X_{2}$, so $\bigcup X_{2}=e$. Also, $f$ is the maximum element of $Y_{2}$, so $\bigcup Y_{2}=f$. Therefore, $\bigcup X_{2}=e=f \cap \nu=\left(\bigcup Y_{2}\right) \cap \nu$, proving the first part of the lemma.

Now suppose $(X, F) *(\check{Y}, \check{K})$ is a condition in $\mathbb{P}_{\nu} * \mathbb{P}\left(\nu, \dot{S}_{\nu}, \beta\right)$ such that $\bigcup X=$ $(\cup Y) \cap \nu$. Let $Z=X \cup Y$ and $L=F \cup K$. By Lemma 5.7, $(Z, L)$ is in $\mathbb{P}_{\beta}, Z_{\nu}=X$, $L_{\nu}=F, Z^{\nu}=Y$, and $L^{\nu}=K$. Also, $\bigcup Z_{\nu}=\bigcup X=(\bigcup Y) \cap \nu=\left(\bigcup Z^{\nu}\right) \cap \nu$, so $(Z, L)$ is in $\mathbb{P}_{\nu, \beta}$.

Theorem 5.9. Define $i: \mathbb{P}_{\nu, \beta} \rightarrow \mathbb{P}_{\nu} * \mathbb{P}\left(\nu, \dot{S}_{\nu}, \beta\right)$ by letting $i(Y, K)=\left(Y_{\nu}, K_{\nu}\right) *$ $\left(\check{Y}^{\nu}, \check{K}^{\nu}\right)$. Then $i$ is an isomorphism of $\mathbb{P}_{\nu, \beta}$ onto a dense subset of $\mathbb{P}_{\nu} * \mathbb{P}\left(\nu, \dot{S}_{\nu}, \beta\right)$.

Proof. By (I) and (III) of Proposition 5.5, if $(Y, K)$ is in $\mathbb{P}_{\nu, \beta}$, then $i(Y, K)$ is in $\mathbb{P}_{\nu} * \mathbb{P}\left(\nu, \dot{S}_{\nu}, \beta\right)$. By (II) and (IV) of Proposition 5.5 , if $(Z, L)$ and $(Y, K)$ are in $\mathbb{P}_{\nu, \beta}$ and $(Z, L) \leq(Y, K)$ in $\mathbb{P}_{\beta}$, then $i(Z, L) \leq i(Y, K)$ in $\mathbb{P}_{\nu} * \mathbb{P}\left(\nu, \dot{S}_{\nu}, \beta\right)$. It is easy to see that $i$ is an injection, and the range of $i$ is dense in $\mathbb{P}_{\nu} * \mathbb{P}\left(\nu, \dot{S}_{\nu}, \beta\right)$ by Lemma 5.8. To complete the proof, it remains to show that if $(Z, L)$ and $(Y, K)$ are in $\mathbb{P}_{\nu, \beta}$ and $i(Z, L) \leq i(Y, K)$ in $\mathbb{P}_{\nu} * \mathbb{P}\left(\nu, \dot{S}_{\nu}, \beta\right)$, then $(Z, L) \leq(Y, K)$ in $\mathbb{P}_{\beta}$.

Let $(Z, L)$ and $(Y, K)$ be in $\mathbb{P}_{\nu, \beta}$, and assume $i(Z, L) \leq i(Y, K)$ in $\mathbb{P}_{\nu} * \mathbb{P}\left(\nu, \dot{S}_{\nu}, \beta\right)$. This means that $\left(Z_{\nu}, L_{\nu}\right) \leq\left(Y_{\nu}, K_{\nu}\right)$ in $\mathbb{P}_{\nu}$, and $\left(Z_{\nu}, L_{\nu}\right) \Vdash_{\mathbb{P}_{\nu}}\left(Z^{\nu}, L^{\nu}\right) \leq\left(Y^{\nu}, K^{\nu}\right)$ in $\mathbb{P}\left(\nu, \dot{S}_{\nu}, \beta\right)$. We prove that $(Z, L) \leq(Y, K)$ in $\mathbb{P}_{\beta}$. 
Since $\left(Z_{\nu}, L_{\nu}\right) \leq\left(Y_{\nu}, K_{\nu}\right)$ in $\mathbb{P}_{\nu}$, we have: (1) $Y_{\nu} \subseteq Z_{\nu}$, (2) for all $b$ in $Z_{\nu} \backslash Y_{\nu}$, $b \nsubseteq \bigcup Y_{\nu},(3) \operatorname{dom}\left(K_{\nu}\right) \subseteq \operatorname{dom}\left(L_{\nu}\right)$, and (4) for all $\xi$ in $\operatorname{dom}\left(K_{\nu}\right), K(\xi)$ is an initial segment of $L(\xi)$. Fix a generic filter $H$ on $\mathbb{P}_{\nu}$ with $\left(Z_{\nu}, L_{\nu}\right)$ in $H$. Then in $V[H]$, $\left(Z^{\nu}, L^{\nu}\right) \leq\left(Y^{\nu}, K^{\nu}\right)$ in $\mathbb{P}\left(\nu, S_{\nu}, \beta\right)$, which means: (5) $Y^{\nu} \subseteq Z^{\nu}$, (6) for all $b$ in $Z^{\nu} \backslash Y^{\nu}, b \nsubseteq \bigcup Y^{\nu},(7) \operatorname{dom}\left(K^{\nu}\right) \subseteq \operatorname{dom}\left(L^{\nu}\right)$, and (8) for all $\xi$ in $\operatorname{dom}\left(K^{\nu}\right), K(\xi)$ is an initial segment of $L(\xi)$. Note that properties (5)-(8) are absolute between $V$ and $V[H]$, so in fact they hold in $V$.

To show that $(Z, L) \leq(Y, K)$ in $\mathbb{P}_{\beta}$, we show (a) $Y \subseteq Z$, (b) for all $b$ in $Z \backslash Y$, $b \nsubseteq \bigcup Y,(\mathrm{c}) \operatorname{dom}(K) \subseteq \operatorname{dom}(L)$, and (d) for all $\xi$ in $\operatorname{dom}(K), K(\xi)$ is an initial segment of $L(\xi)$. Since $Y=Y_{\nu} \cup Y^{\nu}$ and $Z=Z_{\nu} \cup Z^{\nu}$, (a) follows from (1) and (5). Similarly, $K=K_{\nu} \cup K^{\nu}$ and $L=L_{\nu} \cup L^{\nu}$, so (c) and (d) follow from (3), (4), (7), and (8).

It remains to prove (b). Let $b$ be in $Z \backslash Y$, and we show $b \nsubseteq \bigcup Y$. First assume that $b \subseteq \nu$. Then $b$ is in $Z_{\nu} \backslash Y_{\nu}$. By $(2), b \nsubseteq \bigcup Y_{\nu}$. Since $(Y, K)$ is in $\mathbb{P}_{\nu, \beta}$, by

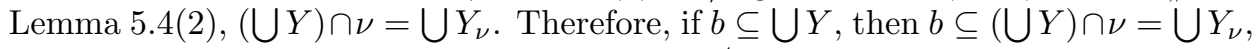
which is a contradiction. Now assume that $b \nsubseteq \nu$. Then $b$ is in $Z^{\nu} \backslash Y^{\nu}$. By (6), $b \nsubseteq \bigcup Y^{\nu}$. As $(Y, K)$ is in $\mathbb{P}_{\nu, \beta}$, by Lemma 5.4(1), $\bigcup Y=\bigcup Y^{\nu}$. Since $b \nsubseteq \bigcup Y^{\nu}$, $b \nsubseteq \bigcup Y$.

As $\mathbb{P}_{\nu, \beta}$ is a dense suborder of $\mathbb{P}_{\beta}$, it follows that $\mathbb{P}_{\beta}$ is forcing equivalent to $\mathbb{P}_{\nu} * \mathbb{P}\left(\nu, \dot{S}_{\nu}, \beta\right)$.

The following is a technical lemma which we will need in the next section.

Lemma 5.10. Let $i: \mathbb{P}_{\nu, \beta} \rightarrow \mathbb{P}_{\nu} * \mathbb{P}\left(\nu, \dot{S}_{\nu}, \beta\right)$ be the map $i(Y, K)=\left(Y_{\nu}, K_{\nu}\right) *$ $\left(\check{Y}^{\nu}, \check{K}^{\nu}\right)$. Suppose $(X, F)$ is a condition in $\mathbb{P}_{\nu}$ (and hence $(X, F)$ is in $\mathbb{P}_{\beta}$ as well). Let $(Y, K)$ be in $\mathbb{P}_{\nu, \beta}$, and assume $(Y, K) \leq(X, F)$ in $\mathbb{P}_{\beta}$. Then $i(Y, K) \leq(X, F) * 1$ in $\mathbb{P}_{\nu} * \mathbb{P}\left(\nu, \dot{S}_{\nu}, \beta\right)$.

Proof. For $(X, F)$ and $(Y, K)$ as above, $i(Y, K) \leq(X, F) * 1$ means $\left(Y_{\nu}, K_{\nu}\right) *$ $\left(\check{Y}^{\nu}, \check{K}^{\nu}\right) \leq(X, F) * 1$, which is equivalent to $\left(Y_{\nu}, K_{\nu}\right) \leq(X, F)$ in $\mathbb{P}_{\nu}$. Therefore, it suffices to show that if $(X, F)$ is in $\mathbb{P}_{\nu},(Y, K)$ is in $\mathbb{P}_{\nu, \beta}$, and $(Y, K) \leq(X, F)$ in $\mathbb{P}_{\beta}$, then $\left(Y_{\nu}, K_{\nu}\right) \leq(X, F)$ in $\mathbb{P}_{\nu}$.

Since $(Y, K) \leq(X, F)$ in $\mathbb{P}_{\beta}, X \subseteq Y$. If $b$ is in $X$, then $b$ is in $Y$, and since $(X, F)$ is in $\mathbb{P}_{\nu}, b$ is a subset of $\nu$. Therefore, $b$ is in $Y_{\nu}$. Hence $X \subseteq Y_{\nu}$. Suppose $b$ is in $Y_{\nu} \backslash X$. Then $b$ is in $Y \backslash X$, so $b \nsubseteq \bigcup X$. As $(Y, K) \leq(X, F)$ in $\mathbb{P}_{\beta}$, $\operatorname{dom}(F) \subseteq \operatorname{dom}(K)$, but also $\operatorname{dom}(F) \subseteq \nu$. Therefore, $\operatorname{dom}(F) \subseteq \operatorname{dom}(K) \cap \nu=$ $\operatorname{dom}\left(K_{\nu}\right)$. If $\xi$ is in $\operatorname{dom}(F)$, then as $(X, F) \leq(Y, K), F(\xi)$ is an initial segment of $K(\xi)=K_{\nu}(\xi)$.

\section{Extending an elementary embedding, Part 1}

We now deal with the problem of extending an elementary embedding after Lévy collapsing a large cardinal to become $\omega_{2}$, and then forcing with $\mathbb{P}_{\omega_{2}}$. For the remainder of the section fix a cardinal $\kappa$ which is $\kappa^{+}$-supercompact, and an elementary embedding $j: V \rightarrow M$ with critical point $\kappa$ such that $j(\kappa)>\kappa^{+}$and $M^{\kappa^{+}} \subseteq M$. Assume $2^{\kappa}=\kappa^{+}$.

Recall that the Lévy collapse $\operatorname{CoLL}\left(\omega_{1},<\kappa\right)$ is $\omega_{1}$-closed, $\kappa$-c.c., has size $\kappa$, and forces that $\kappa$ is equal to $\omega_{2}$. It is also easy to see that $\operatorname{CoLL}\left(\omega_{1},<\kappa\right)$ forces $2^{\omega}=\omega_{1}$ and $2^{\omega_{1}}=\omega_{2}$. Since we are assuming $2^{\kappa}=\kappa^{+}$in $V, \operatorname{ColL}\left(\omega_{1},<\kappa\right)$ forces $2^{\omega_{2}}=\omega_{3}$. We will be interested in the two-step iteration $\operatorname{CoLL}\left(\omega_{1},<\kappa\right) * \dot{\mathbb{P}}_{\kappa}$. 
(Note that $\dot{\mathbb{P}}_{\kappa}$ is the same as $\dot{\mathbb{P}}_{\omega_{2}}$.) Since $\mathrm{CH}$ holds after forcing with the Lévy collapse, this two-step iteration is $\kappa$-c.c. and $\omega_{1}$-distributive. Occasionally we will write $j\left(\operatorname{ColL}\left(\omega_{1},<\kappa\right) * \dot{\mathbb{P}}_{\kappa}\right)$ as $\operatorname{CoLL}\left(\omega_{1},<j(\kappa)\right) * \dot{\mathbb{P}}_{j(\kappa)}$. To reduce superscripts, we will write $\kappa^{+}$to denote the successor of $\kappa$ in $V$, even when working in a generic extension in which this ordinal is no longer a cardinal.

Theorem 6.1. Let $\bar{G} * \bar{H}$ be a generic filter on $j\left(\operatorname{CoLL}\left(\omega_{1},<\kappa\right) * \dot{\mathbb{P}}_{\kappa}\right)$ over $V$. Let $G=\bar{G} \cap \operatorname{CoLL}\left(\omega_{1},<\kappa\right)$ and $H=\bar{H} \cap \mathbb{P}_{\kappa}$. Then $G$ is a generic filter on $\operatorname{ColL}\left(\omega_{1},<\kappa\right)$ over $V, H$ is a generic filter on $\mathbb{P}_{\kappa}$ over $V[G]$, and $j$ can be extended in $V[\bar{G} * \bar{H}]$ to $j: V[G * H] \rightarrow M[\bar{G} * \bar{H}]$ such that $j(G * H)=\bar{G} * \bar{H}$. Moreover:

(1) $M[G * H]^{\kappa^{+}} \cap V[G * H] \subseteq M[G * H]$,

(2) $M[\bar{G} * \bar{H}]$ is equal to $M[\bar{G}][H]\left[H^{\prime}\right]$, where $H^{\prime}$ is a generic filter on $\mathbb{P}\left(\kappa, S_{\kappa}, j(\kappa)\right)$ over $M[\bar{G}][H]$,

(3) $j\left(S_{\kappa}\right)=S_{j(\kappa)}, S_{j(\kappa)}=S_{\kappa} \cup S\left(\kappa, S_{\kappa}, j(\kappa)\right), S_{\kappa}=\left\{b \in S_{j(\kappa)}: b \subseteq \kappa\right\}$, and $S\left(\kappa, S_{\kappa}, j(\kappa)\right)=\left\{b \in S_{j(\kappa)}: b \nsubseteq \kappa\right\}$.

Proof. Since the inclusion map from $\operatorname{CoLL}\left(\omega_{1},<\kappa\right)$ to $\operatorname{CoLL}\left(\omega_{1},<j(\kappa)\right)$ is a complete embedding, $G=\bar{G} \cap \operatorname{CoLL}\left(\omega_{1},<\kappa\right)$ is a generic filter on $\operatorname{CoLL}\left(\omega_{1},<\kappa\right)$ over $V$. As $j\left\lceil\operatorname{CoLt}\left(\omega_{1},<\kappa\right)\right.$ is the identity, $j[G]=G \subseteq \bar{G}$. Therefore, $j$ can be extended to $j: V[G] \rightarrow M[\bar{G}]$ such that $j(G)=\bar{G}$.

As $\operatorname{CoLL}\left(\omega_{1},<\kappa\right)$ is $\kappa$-c.c., $M[G]^{\kappa^{+}} \cap V[G] \subseteq M[G]$. In particular, $\mathbb{P}_{\kappa}$ is computed the same in $V[G]$ and in $M[G]$. By standard facts, $M[\bar{G}]=M[G]\left[G^{\prime}\right]$, where $G^{\prime}=\bar{G} \cap \operatorname{ColL}\left(\omega_{1},[\kappa, j(\kappa))\right)$. Since $\operatorname{CoLL}\left(\omega_{1},[\kappa, j(\kappa))\right)$ is $\omega_{1}$-closed, $\mathbb{P}_{\kappa}$ is computed the same in $M[G]$ and in $M[\bar{G}]$.

In $M[\bar{G}]$ consider the map $i: \mathbb{P}_{\kappa, j(\kappa)} \rightarrow \mathbb{P}_{\kappa} * \mathbb{P}\left(\kappa, \dot{S}_{\kappa}, j(\kappa)\right)$ given by $i(Z, K)=$ $\left(Z_{\kappa}, K_{\kappa}\right) *\left(\check{Z}^{\kappa}, \check{K}^{\kappa}\right)$. By Theorem $5.9, i$ is an isomorphism of $\mathbb{P}_{\kappa, j(\kappa)}$ onto a dense subset of $\mathbb{P}_{\kappa} * \mathbb{P}\left(\kappa, \dot{S}_{\kappa}, j(\kappa)\right)$. However, being an isomorphism is absolute. It follows that $i\left[\bar{H} \cap \mathbb{P}_{\kappa, j(\kappa)}\right]$ generates a generic filter $F$ on $\mathbb{P}_{\kappa} * \mathbb{P}\left(\kappa, \dot{S}_{\kappa}, j(\kappa)\right)$ over $V[\bar{G}]$, and $M[\bar{G}][\bar{H}]=M[\bar{G}][F]$.

Let $k: \mathbb{P}_{\kappa} \rightarrow \mathbb{P}_{\kappa} * \mathbb{P}\left(\kappa, \dot{S}_{\kappa}, j(\kappa)\right)$ be the map $k(p)=p * 1$. Then $k$ is a complete embedding. It follows that $k^{-1}(F)=\left\{p \in \mathbb{P}_{\kappa}: p * 1 \in F\right\}$ is a generic filter on $\mathbb{P}_{\kappa}$ over $V[\bar{G}]$. Let $H=k^{-1}(F)$.

We claim that $H$ is equal to $\bar{H} \cap \mathbb{P}_{\kappa}$. Therefore, let $p$ be in $H$. Then $p$ is in $\mathbb{P}_{\kappa}$, and $p * 1$ is in $F$, the filter generated by $i\left[\bar{H} \cap \mathbb{P}_{\kappa, j(\kappa)}\right]$. Then there is a condition $(Y, K)$ in $\bar{H} \cap \mathbb{P}_{\kappa, j(\kappa)}$ such that $i(Y, K)=\left(Y_{\kappa}, K_{\kappa}\right) *\left(\check{Y}^{\kappa}, \check{K}^{\kappa}\right) \leq p * 1$. This implies $\left(Y_{\kappa}, K_{\kappa}\right) \leq p$ in $\mathbb{P}_{\kappa}$. By Lemma $3.3,\left(Y_{\kappa}, K_{\kappa}\right) \leq p$ in $\mathbb{P}_{j(\kappa)}$. By Lemma $5.6,(Y, K) \leq\left(Y_{\kappa}, K_{\kappa}\right)$ in $\mathbb{P}_{j(\kappa)}$, so $(Y, K) \leq p$ in $\mathbb{P}_{j(\kappa)}$. Since $(Y, K)$ is in $\bar{H}, p$ is in $\bar{H}$. This shows $H \subseteq \bar{H} \cap \mathbb{P}_{\kappa}$.

On the other hand, let $q$ be in $\bar{H} \cap \mathbb{P}_{\kappa}$, and we show $q$ is in $H$. In other words, we show $q * 1$ is in $F$. Since $\mathbb{P}_{\kappa, j(\kappa)}$ is dense in $\mathbb{P}_{j(\kappa)}$ and $q$ is in $\bar{H}$, choose $q^{\prime} \leq q$ in $\bar{H} \cap \mathbb{P}_{\kappa, j(\kappa)}$. Then $i\left(q^{\prime}\right) \in i\left[\bar{H} \cap \mathbb{P}_{\kappa, j(\kappa)}\right]$, so $i\left(q^{\prime}\right)$ is in $F$. Since $q^{\prime} \leq q$ in $\mathbb{P}_{j(\kappa)}$ and $q$ is in $\mathbb{P}_{\kappa}$, by Lemma $5.10, i\left(q^{\prime}\right) \leq q * 1$ in $\mathbb{P}_{\kappa} * \mathbb{P}\left(\kappa, \dot{S}_{\kappa}, j(\kappa)\right)$. As $i\left(q^{\prime}\right)$ is in $F, q * 1$ is in $F$. This completes the proof that $H=\bar{H} \cap \mathbb{P}_{\kappa}$.

Now $j\left\lceil\mathbb{P}_{\kappa}\right.$ is the identity, as can be easily seen, so $j[H]=H=\bar{H} \cap \mathbb{P}_{\kappa} \subseteq \bar{H}$. Therefore, we can extend $j$ to $j: V[G][H] \rightarrow M[\bar{G}][\bar{H}]$ such that $j(H)=\bar{H}$, so $j(G * H)=j(G) * j(H)=\bar{G} * \bar{H}$. Since $\operatorname{Colv}\left(\omega_{1},<\kappa\right) * \mathbb{P}_{\kappa}$ is $\kappa$-c.c., $M[G * H]^{\kappa^{+}} \cap$ $V[G * H] \subseteq M[G * H]$. 
Recall that $M[\bar{G}][\bar{H}]=M[\bar{G}][F]$, where $F$ is a generic filter on $\mathbb{P}_{\kappa} * \mathbb{P}\left(\kappa, \dot{S}_{\kappa}, j(\kappa)\right)$ over $M[\bar{G}]$. By standard facts about two-step iterations, if we let $H^{\prime}=\left\{\dot{s}^{H}\right.$ : $\exists p p * \dot{s} \in F\}$, then $H^{\prime}$ is a generic filter on $\mathbb{P}\left(\kappa, S_{\kappa}, j(\kappa)\right)$ over $M[\bar{G}][H]$, and $M[\bar{G}][F]=M[\bar{G}][H]\left[H^{\prime}\right]$. Hence, $M[\bar{G}][\bar{H}]=M[\bar{G}][H]\left[H^{\prime}\right]$.

It is immediate by the elementarity of $j$ and the fact that $j(H)=\bar{H}$ that $j\left(S_{\kappa}\right)=S_{j(\kappa)}$. If $b$ is in $S_{\kappa}$, then by elementarity, $j(b)=b$ is in $j\left(S_{\kappa}\right)=S_{j(\kappa)}$. Therefore, $S_{\kappa} \subseteq S_{j(\kappa)}$.

By definition, $S\left(\kappa, S_{\kappa}, j(\kappa)\right)$ is equal to $\bigcup\left\{Y: \exists K(Y, K) \in H^{\prime}\right\}$. As a subclaim, let us prove that $S\left(\kappa, S_{\kappa}, j(\kappa)\right)$ is equal to $\bigcup\left\{Z^{\kappa}: \exists L(Z, L) \in \bar{H} \cap \mathbb{P}_{\kappa, j(\kappa)}\right\}$.

On the one hand, suppose $b$ is in $Z^{\kappa}$, where for some $L,(Z, L)$ is in $\bar{H} \cap \mathbb{P}_{\kappa, j(\kappa)}$. Then $i(Z, L)=\left(Z_{\kappa}, L_{\kappa}\right) *\left(\check{Z}^{\kappa}, \check{L}^{\kappa}\right)$ is in $F$. By the definition of $H^{\prime},\left(Z^{\kappa}, L^{\kappa}\right)$ is in $H^{\prime}$, so $Z^{\kappa} \subseteq S\left(\kappa, S_{\kappa}, j(\kappa)\right)$. Hence $b$ is in $S\left(\kappa, S_{\kappa}, j(\kappa)\right)$.

On the other hand, suppose $b$ is in $S\left(\kappa, S_{\kappa}, j(\kappa)\right)$. Then there is $(Y, K)$ in $H^{\prime}$ such that $b$ is in $Y$. By the definition of $H^{\prime}$, fix $p * \dot{s}$ in $F$ such that $\dot{s}^{H}=(Y, K)$. Since $p * \dot{s}$ is in $F$, there is $(Z, L)$ in $\bar{H} \cap \mathbb{P}_{\kappa, j(\kappa)}$ such that $i(Z, L)=\left(Z_{\kappa}, L_{\kappa}\right) *\left(\check{Z}^{\kappa}, \check{L}^{\kappa}\right) \leq p * \dot{s}$. By Lemma 5.6, $(Z, L) \leq\left(Z_{\kappa}, L_{\kappa}\right)$ in $\mathbb{P}_{j(\kappa)}$, so $\left(Z_{\kappa}, L_{\kappa}\right)$ is in $\bar{H} \cap \mathbb{P}_{\kappa}=H$. Since $\left(Z_{\kappa}, L_{\kappa}\right) *\left(\check{Z}^{\kappa}, \check{L}^{\kappa}\right) \leq p * \dot{s}$ and $\left(Z_{\kappa}, L_{\kappa}\right) \in H,\left(Z^{\kappa}, L^{\kappa}\right) \leq \dot{s}^{H}=(Y, K)$. In particular, $Y \subseteq Z^{\kappa}$. Therefore, $b$ is in $Z^{\kappa}$. This proves $S\left(\kappa, S_{\kappa}, j(\kappa)\right) \subseteq \bigcup\left\{Z^{\kappa}: \exists L(Z, L) \in\right.$ $\left.\bar{H} \cap \mathbb{P}_{\kappa, j(\kappa)}\right\}$, finishing the proof of the subclaim.

Let us prove that $S_{j(\kappa)}=S_{\kappa} \cup S\left(\kappa, S_{\kappa}, j(\kappa)\right)$. We already observed $S_{\kappa} \subseteq S_{j(\kappa)}$. Let $b$ be in $S\left(\kappa, S_{\kappa}, j(\kappa)\right)$. By the subclaim, there is $(Z, L)$ in $\bar{H} \cap \mathbb{P}_{\kappa, j(\kappa)}$ such that $b$ is in $Z^{\kappa}$. Since $(Z, L)$ is in $\bar{H}, Z \subseteq S_{j(\kappa)}$. However, $b$ is in $Z^{\kappa} \subseteq Z$, so $b$ is in $S_{j(\kappa)}$.

Now let $b$ be in $S_{j(\kappa)}$, and we show that $b$ is in $S_{\kappa} \cup S\left(\kappa, S_{\kappa}, j(\kappa)\right)$. Fix $(Y, K)$ in $\bar{H}$ such that $b$ is in $Y$. Since $\mathbb{P}_{\kappa, j(\kappa)}$ is dense in $\mathbb{P}_{j(\kappa)}$, fix $(Z, L) \leq(Y, K)$ in $\bar{H} \cap \mathbb{P}_{\kappa, j(\kappa)}$. Then $b$ is in $Z$. By the subclaim, $Z^{\kappa} \subseteq S\left(\kappa, S_{\kappa}, j(\kappa)\right)$. Therefore, if $b$ is in $Z^{\kappa}$, then $b$ is in $S\left(\kappa, S_{\kappa}, j(\kappa)\right)$. Otherwise $b \subseteq \kappa$, so $b$ is in $Z_{\kappa}$. By Lemma 5.6, $(Z, L) \leq\left(Z_{\kappa}, L_{\kappa}\right)$ in $\mathbb{P}_{j(\kappa)}$, so $\left(Z_{\kappa}, L_{\kappa}\right)$ is in $\bar{H} \cap \mathbb{P}_{\kappa}=H$. This implies $Z_{\kappa} \subseteq S_{\kappa}$, and hence $b$ is in $S_{\kappa}$.

We have proven $S_{j(\kappa)}=S_{\kappa} \cup S\left(\kappa, S_{\kappa}, j(\kappa)\right)$. Now every set in $S_{\kappa}$ is a subset of $\kappa$, and every set in $S\left(\kappa, S_{\kappa}, j(\kappa)\right)$ is not a subset of $\kappa$. Therefore, it is immediate that $S_{\kappa}=\left\{b \in S_{j(\kappa)}: b \subseteq \kappa\right\}$ and $S\left(\kappa, S_{\kappa}, j(\kappa)\right)=\left\{b \in S_{j(\kappa)}: b \nsubseteq \kappa\right\}$.

\section{Adding a Club}

We briefly describe a forcing poset due to Sakai 3 . for adding a club subset of $P_{\omega_{1}}\left(\omega_{2}\right)$. Specifically, for a given set $T \subseteq P_{\omega_{1}}\left(\omega_{2}\right)$, we define a forcing poset $\mathbb{Q}(T)$ which adds a generic function $F: \omega_{2} \times \omega_{2} \rightarrow \omega_{1}$ for which there is no set in $T$ closed under $F$. In the next section we describe an iteration of this forcing poset.

Definition 7.1. Let $T$ be a subset of $P_{\omega_{1}}\left(\omega_{2}\right)$. Define $\mathbb{Q}(T)$ as the forcing poset consisting of conditions $p$ satisfying:

- $p$ is a function of the form $p: a^{p} \times a^{p} \rightarrow \omega_{1}$, where $a^{p}$ is a countable subset of $\omega_{2}$,

- for all $x$ in $T$, if $x \subseteq a^{p}$, then $x$ is not closed under $p$.

Let $q \leq p$ if $q$ extends $p$ as a function, that is, if $a^{p} \subseteq a^{q}$ and $q \uparrow\left(a^{p} \times a^{p}\right)=p$.

Notation 7.2. For a countable set $a \subseteq \omega_{2}$, we write $a^{2}$ for $a \times a$. 
Lemma 7.3. Let $p$ and $q$ be conditions in $\mathbb{Q}(T)$, and assume $p\left\lceil\left(a^{p} \cap a^{q}\right)^{2}=q \uparrow\right.$ $\left(a^{p} \cap a^{q}\right)^{2}$. Then $p$ and $q$ are compatible.

In particular, if $a^{p} \cap a^{q}=\emptyset$, then $p$ and $q$ are compatible.

Proof. We define a condition $r$ which is below $p$ and $q$. Let $a^{r}=a^{p} \cup a^{q}$. As $a^{r}$ is countable, fix an ordinal $\zeta$ in $\omega_{1} \backslash a^{r}$. Define $r:\left(a^{r}\right)^{2} \rightarrow \omega_{1}$ as follows. Let $(i, j)$ be in $\left(a^{r}\right)^{2}$. If $i$ and $j$ are both in $a^{p}$, let $r(i, j)=p(i, j)$. If $i$ and $j$ are both in $a^{q}$, let $r(i, j)=q(i, j)$. Note this is well-defined, since if $i$ and $j$ are both in $a^{p} \cap a^{q}$, then $p(i, j)=q(i, j)$ by assumption. The remaining possibility is that $i$ is in $a^{p} \backslash a^{q}$ and $j$ is in $a^{q} \backslash a^{p}$, or vice versa. In this case, let $r(i, j)=\zeta$.

Therefore, $r$ is a function of the form $r:\left(a^{r}\right)^{2} \rightarrow \omega_{1}$, where $a^{r}$ is a countable subset of $\omega_{2}$. Note that $r$ extends both $p$ and $q$. To show $r$ is a condition, suppose $x$ is in $T$ and $x \subseteq a^{r}$. If $x$ is a subset of $a^{p}$, then $x$ is not closed under $p$, since $p$ is a condition. Hence, $x$ is not closed under $r$. Likewise, if $x$ is a subset of $a^{q}$, then $x$ is not closed under $q$, so $x$ is not closed under $r$. If neither of the above holds, then there is $i$ in $a^{r} \backslash a^{q}$ and $j$ in $a^{r} \backslash a^{p}$. But then $i$ is in $a^{p} \backslash a^{q}$ and $j$ is in $a^{q} \backslash a^{p}$. By the definition of $r, r(i, j)=\zeta$. Since $\zeta$ is not in $a^{r}, \zeta$ is not in $x$, so $x$ is not closed under $r$.

Lemma 7.4. Let $b$ be a countable subset of $\omega_{2}$. Then there are densely many conditions $t$ in $\mathbb{Q}(T)$ such that $b \subseteq a^{t}$.

Proof. Let $p:\left(a^{p}\right)^{2} \rightarrow \omega_{1}$ be a given condition. If $b \subseteq a^{p}$, then we are done. Otherwise $b \backslash a^{p}$ is non-empty. As $b$ is countable, fix an ordinal $\zeta$ in $\omega_{1} \backslash b$. Define $s$ by letting $a^{s}=b \backslash a^{p}$, and for all $(i, j)$ in $\left(a^{s}\right)^{2}$, let $s(i, j)=\zeta$. If $x$ is in $T$ and is a subset of $a^{s}$, then $\zeta$ is not in $x$, so $x$ is not closed under $s$. This shows $s$ is in $\mathbb{Q}(T)$. Now $a^{s} \cap a^{p}=\emptyset$, so by Lemma 7.3,s and $p$ are compatible. Let $t \leq s, p$. Then $b \subseteq a^{s} \cup a^{p} \subseteq a^{t}$.

Suppose $I$ is a generic filter on $\mathbb{Q}(T)$ over $V$. Then by the last lemma, it is easy to see that $F=\bigcup I$ is a total function $F: \omega_{2}^{V} \times \omega_{2}^{V} \rightarrow \omega_{1}^{V}$, and if $b$ is in $T$, then $b$ is not closed under $F$. Therefore, if $\omega_{1}$ and $\omega_{2}$ are preserved, then $\mathbb{Q}(T)$ destroys the stationarity of $T$.

Depending on the assumptions of the ground model and on the choice of $T, \mathbb{Q}(T)$ may or may not preserve cardinals. In the sections which follow, we will define and analyze an iteration of forcing posets of the form $\mathbb{Q}(T)$. In our context, the forcing posets $\mathbb{Q}(T)$ which we use, together with the iteration itself, are $\omega_{1}$-distributive and $\omega_{2}$-c.c.

\section{ITERATION OF CLUB ADDING}

In this section we define an iteration of forcing posets of the form $\mathbb{Q}(T)$ from the previous section. We work in a model $V[H]$, where $H$ is a generic filter on $\mathbb{P}_{\omega_{2}}$ over $V$. As usual, let $S_{\omega_{2}}=\bigcup\{X: \exists F(X, F) \in H\}$. Therefore, $S_{\omega_{2}}$ is a stationary subset of $P_{\omega_{1}}\left(\omega_{2}\right)$ in $V[H]$ which does not reflect to any ordinal in $\omega_{2}$ with cofinality $\omega_{1}$.

Our goal is to define a forcing poset $\mathbb{Q}$ which forces that every stationary subset of $S_{\omega_{2}}$ reflects to an uncountable ordinal in $\omega_{2}$ with cofinality $\omega$. The forcing poset $\mathbb{Q}$ will be an iteration of forcing posets of the form $\mathbb{Q}(T)$, where $T$ is a subset of $S_{\omega_{2}}$ which does not reflect to any uncountable ordinal in $\omega_{2}$ of cofinality $\omega$.

The iteration we define is an adaptation of an iteration developed by Sakai, which he used to prove the following theorem. 
Theorem 8.1 (Sakai, [3]). Assume GCH and $\square_{\omega_{1}}$. Then there exists a forcing poset which forces that there is a stationary set $S \subseteq P_{\omega_{1}}\left(\omega_{2}\right)$ such that every stationary subset of $S$ reflects to an uncountable ordinal in $\omega_{2}$ with cofinality $\omega$.

Let us describe in more detail the context in which we will define $\mathbb{Q}$. We start with a ground model $V$ which satisfies $2^{\omega}=\omega_{1}, 2^{\omega_{1}}=\omega_{2}$, and $2^{\omega_{2}}=\omega_{3}$. Let $H$ be a generic filter on $\mathbb{P}_{\omega_{2}}$ over $V$. Let $S_{\omega_{2}}=\bigcup\{X: \exists F(X, F) \in H\}$.

We verify that in $V[H]$ it is still the case that $2^{\omega}=\omega_{1}, 2^{\omega_{1}}=\omega_{2}$, and $2^{\omega_{2}}=\omega_{3}$. Since $\mathbb{P}_{\omega_{2}}$ is $\omega_{1}$-distributive, $\mathbb{P}_{\omega_{2}}$ does not add any new subsets of $\omega$. Therefore, $2^{\omega}=\omega_{1}$ in $V[H]$. As $\mathrm{CH}$ holds in $V$, it is easy to see that $\mathbb{P}_{\omega_{2}}$ has cardinality $\omega_{2}$. Since $\mathbb{P}_{\omega_{2}}$ is $\omega_{2}$-c.c., it has at most $\omega_{2}^{\omega_{1}}=2^{\omega_{1}}=\omega_{2}$ many antichains. Therefore, the number of nice $\mathbb{P}_{\omega_{2}}$-names in $V$ for a subset of $\omega_{1}$ is bounded by $\omega_{2}^{\omega_{1}}=2^{\omega_{1}}=\omega_{2}$. It follows that $2^{\omega_{1}}=\omega_{2}$ in $V[H]$. Similarly, the number of nice $\mathbb{P}_{\omega_{2}}$-names in $V$ for a subset of $\omega_{2}$ is $\omega_{2}^{\omega_{2}}=2^{\omega_{2}}=\omega_{3}$, so $2^{\omega_{2}}=\omega_{3}$ in $V[H]$.

We will work in $V[H]$ to define a forcing iteration $\mathbb{Q}$ which destroys the stationarity of any subset of $S_{\omega_{2}}$ which does not reflect to an uncountable ordinal in $\omega_{2}$ with cofinality $\omega$. Actually, we will find it convenient to define $\mathbb{Q}$ in a way that is not literally a forcing iteration, although it is forcing equivalent to one.

Before beginning the definition of $\mathbb{Q}$, we fix several auxiliary objects which will be needed in the definition. Since we will remind the reader of these objects later in the paper, we provide them with numbers for reference.

Notation 8.2. For the purposes of bookkeeping, fix a surjective function $f: \omega_{3} \rightarrow$ $\omega_{3} \times \omega_{3}$ satisfying that whenever $f(\alpha)=(i, j)$, then $i \leq \alpha$.

It will be convenient to have at hand an auxiliary poset $\mathbb{R}$, which will be useful for notational purposes. However, we will not be interested in forcing with $\mathbb{R}$.

Notation 8.3. Let $\mathbb{R}$ denote the set of all non-empty partial functions $s$ of the form $s:\left(a^{s}\right)^{2} \rightarrow \omega_{1}$, where $a^{s}$ is a countable subset of $\omega_{2}$. Let $t \leq_{\mathbb{R}} s$ if $t$ extends $s$ as a function, that is, $a^{s} \subseteq a^{t}$ and $t \uparrow\left(a^{s}\right)^{2}=s$.

Note that by $\mathrm{CH}, \mathbb{R}$ has cardinality $\omega_{2}$.

Notation 8.4. For each ordinal $\alpha$ in $\omega_{2}$, fix a surjective function $g_{\alpha}: \omega_{1} \rightarrow \alpha$. Let $E$ be the set of $b$ in $P_{\omega_{1}}\left(\omega_{2}\right)$ such that ot $(b)$ is a limit ordinal, and for all $\alpha$ in $b, b$ is closed under $g_{\alpha}$. Note that $E$ is a club subset of $P_{\omega_{1}}\left(\omega_{2}\right)$.

We are now ready to define by recursion the forcing poset $\mathbb{Q}$ in the model $V[H]$. Specifically, we define by recursion sequences $\left\langle\mathbb{Q}_{\alpha}: \alpha \leq \omega_{3}\right\rangle$ and $\left\langle\dot{T}_{j}^{i}: i, j \leq \omega_{3}\right\rangle$. For each $\alpha<\omega_{3}$, we let $\dot{T}(\alpha)$ be $\dot{T}_{j}^{i}$, where $f(\alpha)=(i, j)$. Finally, the forcing poset $\mathbb{Q}$ is defined as $\mathbb{Q}_{\omega_{3}}$.

We will maintain several recursion hypotheses.

Recursion hypotheses 8.5. For all $\alpha \leq \omega_{3}$ :

(1) If $p$ is in $\mathbb{Q}_{\alpha}$, then $p$ is a partial function $p: \alpha \rightarrow \mathbb{R}$ with a countable domain, and for all $p$ and $q$ in $\mathbb{Q}_{\alpha}, q \leq p$ iff $\operatorname{dom}(p) \subseteq \operatorname{dom}(q)$, and for all $\nu$ in $\operatorname{dom}(p), q(\nu) \leq_{\mathbb{R}} p(\nu)$.

(2) Let $\beta<\alpha$. Then: (a) for all $q$ in $\mathbb{Q}_{\alpha}, q \uparrow \beta$ is in $\mathbb{Q}_{\beta}$, (b) $\mathbb{Q}_{\beta} \subseteq \mathbb{Q}_{\alpha}$, (c) if $q$ is in $\mathbb{Q}_{\alpha}$ and $s \leq q \uparrow \beta$ in $\mathbb{Q}_{\beta}$, then letting $t=s \cup(q \uparrow[\beta, \alpha))$, $t$ is in $\mathbb{Q}_{\alpha}$ and $t \leq s, q$ in $\mathbb{Q}_{\alpha}$, and (d) the inclusion map $\mathbb{Q}_{\beta} \rightarrow \mathbb{Q}_{\alpha}$ is a complete embedding. 
(3) $\mathbb{Q}_{\alpha}$ is $\omega_{1}$-distributive and $\omega_{2}$-c.c.

(4) If $\alpha<\omega_{3}$, then the sequence $\left\langle\dot{T}_{i}^{\alpha}: i<\omega_{3}\right\rangle$ is an enumeration of all nice $\mathbb{Q}_{\alpha}$-names $\dot{T}$ for a subset of $P_{\omega_{1}}\left(\omega_{2}\right)$ satisfying the property that $\mathbb{Q}_{\alpha}$ forces $\dot{T} \subseteq S_{\omega_{2}} \cap E$ and $\dot{T}$ does not reflect to any uncountable ordinal in $\omega_{2}$ with cofinality $\omega$.

Let $\alpha \leq \omega_{3}$ be given, and assume the sequences $\left\langle\mathbb{Q}_{i}: i<\alpha\right\rangle$ and $\left\langle\dot{T}_{i}^{\beta}: \beta<\alpha, i<\right.$ $\left.\omega_{3}\right\rangle$ are defined and satisfy the recursion hypotheses.

If $\alpha<\omega_{3}$ and we have defined $\mathbb{Q}_{\alpha}$ which satisfies recursion hypotheses (1), (2), and (3), then recursion hypothesis (4) is straightforward. This is clear if $\alpha=0$. Suppose $\alpha>0$. By $\mathrm{CH}$ the poset $\mathbb{R}$ has cardinality $\omega_{2}$, so the collection of all partial functions $p: \alpha \rightarrow \mathbb{R}$ with a countable domain has cardinality $\omega_{2}$. Since $\mathbb{Q}_{\alpha}$ is a subset of this collection by recursion hypothesis (1), $\mathbb{Q}_{\alpha}$ has cardinality at most $\omega_{2}$. By recursion hypothesis $(3), \mathbb{Q}_{\alpha}$ is $\omega_{2}$-c.c., so the number of antichains of $\mathbb{Q}_{\alpha}$ is at most $\omega_{2}^{\omega_{1}}=\omega_{2}$. Hence the number of nice $\mathbb{Q}_{\alpha}$-names for a subset of $P_{\omega_{1}}\left(\omega_{2}\right)$ is at most $\omega_{2}^{\omega_{2}}=\omega_{3}$. Consequently, it is possible to choose an enumeration $\left\langle\dot{T}_{i}^{\alpha}: i<\omega_{3}\right\rangle$ satisfying recursion hypothesis (4).

Now we define $\mathbb{Q}_{\alpha}$. The definition splits into three cases.

\section{Case 1. $\boldsymbol{\alpha}$ is equal to $\mathbf{0 .}$}

Let $\mathbb{Q}_{0}$ be the trivial forcing poset consisting of just the empty function. Note that recursion hypotheses (1), (2), and (3) are trivially true.

\section{Case 2. $\alpha$ is a limit ordinal.}

Let $\mathbb{Q}_{\alpha}$ consist of all partial functions $p: \alpha \rightarrow \mathbb{R}$ with a countable domain which satisfy the property that for all $\beta<\alpha, p\left\lceil\beta\right.$ is in $\mathbb{Q}_{\beta}$. Let $q \leq p$ in $\mathbb{Q}_{\alpha}$ if $\operatorname{dom}(p) \subseteq \operatorname{dom}(q)$, and for all $\nu$ in $\operatorname{dom}(p), q(\nu) \leq \mathbb{R} p(\nu)$.

Note that recursion hypothesis (1) is immediate.

\section{Case 3. $\alpha$ is a successor ordinal.}

Let $\alpha=\alpha_{0}+1$. Recall our bookkeeping function $f$ from Notation 8.2. Let $f\left(\alpha_{0}\right)=(i, j)$. Then $i \leq \alpha_{0}$ and $j<\omega_{3}$, so $\dot{T}_{j}^{i}$ is defined and is equal to $\dot{T}\left(\alpha_{0}\right)$.

By recursion hypothesis $(4), \dot{T}\left(\alpha_{0}\right)$ is a nice $\mathbb{Q}_{i}$-name for a subset of $P_{\omega_{1}}\left(\omega_{2}\right)$. Since the inclusion map $\mathbb{Q}_{i} \rightarrow \mathbb{Q}_{\alpha_{0}}$ is a complete embedding by recursion hypothesis (2), $\dot{T}\left(\alpha_{0}\right)$ is a nice $\mathbb{Q}_{\alpha_{0}}$-name for a subset of $P_{\omega_{1}}\left(\omega_{2}\right)$. Also by $(4), \mathbb{Q}_{i}$ forces that $\dot{T}\left(\alpha_{0}\right)$ is a subset of $S_{\omega_{2}} \cap E$ which does not reflect to any uncountable ordinal in $\omega_{2}$ with cofinality $\omega$. But this property of $\dot{T}\left(\alpha_{0}\right)$ is upwards absolute between a generic extension by $\mathbb{Q}_{i}$ and a generic extension by $\mathbb{Q}_{\alpha_{0}}$. Therefore, $\mathbb{Q} \alpha_{0}$ forces that $\dot{T}\left(\alpha_{0}\right)$ is a subset of $S_{\omega_{2}} \cap E$ which does not reflect to any uncountable ordinal in $\omega_{2}$ with cofinality $\omega$.

Let $\mathbb{Q}_{\alpha}$ be the set of all partial functions $p: \alpha \rightarrow \mathbb{R}$ with a countable domain which satisfy that $p\left\lceil\alpha_{0}\right.$ is in $\mathbb{Q}_{\alpha_{0}}$, and if $\alpha_{0}$ is in $\operatorname{dom}(p)$, then $p\left\lceil\alpha_{0} \Vdash_{\mathbb{Q}_{\alpha_{0}}} p\left(\alpha_{0}\right) \in\right.$ $\mathbb{Q}\left(\dot{T}\left(\alpha_{0}\right)\right)$. Let $q \leq p$ if $\operatorname{dom}(p) \subseteq \operatorname{dom}(q)$, and for all $\nu$ in $\operatorname{dom}(p), q(\nu) \leq_{\mathbb{R}} p(\nu)$.

Note that recursion hypothesis (1) is immediate.

Lemma 8.6. Let $p$ and $q$ be in $\mathbb{Q}_{\alpha_{0}+1}$. Then $q \leq p$ iff $q\left\lceil\alpha_{0} \leq p\left\lceil\alpha_{0}\right.\right.$ in $\mathbb{Q}_{\alpha_{0}}$, and if $\alpha_{0}$ is in $\operatorname{dom}(p)$, then $\alpha_{0}$ is in $\operatorname{dom}(q)$ and $q\left(\alpha_{0}\right) \leq_{\mathbb{R}} p\left(\alpha_{0}\right)$.

The proof is straightforward, using recursion hypothesis (1) applied to $\mathbb{Q}_{\alpha_{0}}$.

Lemma 8.7. The forcing poset $\mathbb{Q}_{\alpha_{0}+1}$ is isomorphic to a dense subset of $\mathbb{Q}_{\alpha_{0}}$ * $\mathbb{Q}\left(\dot{T}\left(\alpha_{0}\right)\right)$. Hence, $\mathbb{Q}_{\alpha_{0}+1}$ and $\mathbb{Q}_{\alpha_{0}} * \mathbb{Q}\left(\dot{T}\left(\alpha_{0}\right)\right)$ are forcing equivalent. 
Proof. Define $i: \mathbb{Q}_{\alpha_{0}+1} \rightarrow \mathbb{Q}_{\alpha_{0}} * \mathbb{Q}\left(\dot{T}\left(\alpha_{0}\right)\right)$ by letting $i(p)=\left(p\left\lceil\alpha_{0}\right) * \dot{1}\right.$ if $\alpha_{0}$ is not in $\operatorname{dom}(p)$, and $i(p)=\left(p\left\lceil\alpha_{0}\right) * p\left(\alpha_{0}\right)\right.$ if $\alpha_{0}$ is in $\operatorname{dom}(p)$. By the definition of $\mathbb{Q}_{\alpha_{0}+1}$, it is immediate that $i(p)$ is in $\mathbb{Q}_{\alpha_{0}} * \mathbb{Q}\left(\dot{T}\left(\alpha_{0}\right)\right)$.

Clearly $i$ is an injection. Note that $\mathbb{Q}_{\alpha_{0}}$ forces that for all $a$ and $b$ in $\mathbb{Q}\left(\dot{T}\left(\alpha_{0}\right)\right)$, $a$ and $b$ are in $\mathbb{R}$, and $b \leq a$ in $\mathbb{Q}\left(\dot{T}\left(\alpha_{0}\right)\right)$ iff $b \leq_{\mathbb{R}} a$. Using this observation and Lemma 8.6, it is routine to verify that for all $p$ and $q$ in $\mathbb{Q}_{\alpha_{0}+1}, q \leq p$ iff $i(q) \leq i(p)$.

It remains to show that the range of $i$ is dense. Let $p * \dot{s}$ be in $\mathbb{Q}_{\alpha_{0}} * \mathbb{Q}\left(\dot{T}\left(\alpha_{0}\right)\right)$. By recursion hypothesis (3), $\mathbb{Q}_{\alpha_{0}}$ is $\omega_{1}$-distributive, so $\mathbb{Q}_{\alpha_{0}}$ forces $\dot{s}$ is in the ground model. Hence we can find a set $s$ and a condition $q \leq p$ in $\mathbb{Q}_{\alpha_{0}}$ such that $q \Vdash \check{s}=\dot{s}$. Let $r=q \cup\left\{\left(\alpha_{0}, s\right)\right\}$ if $s$ is non-empty and $r=q$ if $s$ is empty. Then $r$ is in $\mathbb{Q}_{\alpha_{0}+1}$ and $i(r)=q * \check{s} \leq p * \dot{s}$.

This completes the recursive definition.

We have taken care of recursion hypotheses (1) and (4). Proving that $\mathbb{Q}_{\alpha}$ is $\omega_{1}$-distributive and $\omega_{2}$-c.c. will take some work, and we postpone it until the next section. The proof of recursion hypothesis (2) is routine, but we include it for completeness.

Lemma 8.8. Let $\beta<\alpha$. Then:

(a) for all $q$ in $\mathbb{Q}_{\alpha}, q \uparrow \beta \in \mathbb{Q}_{\beta}$,

(b) $\mathbb{Q}_{\beta} \subseteq \mathbb{Q}_{\alpha}$,

(c) if $q$ is in $\mathbb{Q}_{\alpha}$ and $s \leq q\left\lceil\beta\right.$ in $\mathbb{Q}_{\beta}$, then letting $t=s \cup(q\lceil[\beta, \alpha))$, $t$ is in $\mathbb{Q}_{\alpha}$ and $t \leq s, q$ in $\mathbb{Q}_{\alpha}$,

(d) the inclusion map $\mathbb{Q}_{\beta} \rightarrow \mathbb{Q}_{\alpha}$ is a complete embedding.

Proof. (a) Let $q$ be in $\mathbb{Q}_{\alpha}$. If $\alpha$ is a limit ordinal, then $q \uparrow \beta$ is in $\mathbb{Q}_{\beta}$ by the definition of $\mathbb{Q}_{\alpha}$. Otherwise $\alpha$ is a successor ordinal. Let $\alpha=\alpha_{0}+1$. If $\beta=\alpha_{0}$, then $q\left\lceil\alpha_{0}\right.$ is in $\mathbb{Q}_{\alpha_{0}}$ by the definition of $\mathbb{Q}_{\alpha}$. If $\beta<\alpha_{0}$, then $q\lceil\beta$ is equal to $\left(q\left\lceil\alpha_{0}\right) \uparrow \beta\right.$, which is in $\mathbb{Q}_{\beta}$ by recursion hypothesis (2)(a) applied to $\mathbb{Q}_{\alpha_{0}}$.

(b) First suppose $\alpha$ is a successor ordinal. Let $\alpha=\alpha_{0}+1$. Note that by the definition of $\mathbb{Q}_{\alpha}, \mathbb{Q}_{\alpha_{0}} \subseteq \mathbb{Q}_{\alpha}$. By recursion hypothesis (2)(b) applied to $\mathbb{Q}_{\alpha_{0}}$, $\mathbb{Q}_{\beta} \subseteq \mathbb{Q}_{\alpha_{0}}$. Therefore, $\mathbb{Q}_{\beta} \subseteq \mathbb{Q}_{\alpha}$. Now assume $\alpha$ is a limit ordinal, and let $p$ be in $\mathbb{Q}_{\beta}$. To show $p$ is in $\mathbb{Q}_{\alpha}$, we show that $p$ is a partial function $p: \alpha \rightarrow \mathbb{R}$ with a countable domain, and for all $\gamma<\alpha, p\left\lceil\gamma\right.$ is in $\mathbb{Q}_{\gamma}$. The first statement follows from recursion hypothesis (1) applied to $\mathbb{Q}_{\beta}$. Now let $\gamma<\alpha$, and we show $p\lceil\gamma$ is in $\mathbb{Q}_{\gamma}$. If $\gamma<\beta$, then $p\left\lceil\gamma\right.$ is in $\mathbb{Q}_{\gamma}$ by recursion hypothesis (2)(a) applied to $\mathbb{Q}_{\beta}$. If $\beta \leq \gamma$, then $p\left\lceil\gamma=p\right.$, and by recursion hypothesis (2)(b) applied to $\mathbb{Q}_{\gamma}, p$ is in $\mathbb{Q}_{\gamma}$.

(c) Let $q$ be in $\mathbb{Q}_{\alpha}$, and suppose $s \leq q\left\lceil\beta\right.$ in $\mathbb{Q}_{\beta}$. Let $t=s \cup(q \uparrow[\beta, \alpha))$. We will prove $t$ is in $\mathbb{Q}_{\alpha}$. It is then easy to verify that $t \leq s, q$ in $\mathbb{Q}_{\alpha}$. First assume $\alpha$ is a limit ordinal. Clearly $t$ is a partial function $t: \alpha \rightarrow \mathbb{R}$ with a countable domain. Let $\gamma<\alpha$, and we show $t \uparrow \gamma$ is in $\mathbb{Q}_{\gamma}$. If $\gamma \leq \beta$, then $t \uparrow \gamma=s \uparrow \gamma$, which is in $\mathbb{Q}_{\gamma}$ by recursion hypothesis (2)(a) applied to $\mathbb{Q}_{\beta}$. Suppose $\beta<\gamma$. Let $\bar{q}=q \uparrow \gamma$. Then by (a), $\bar{q}$ is in $\mathbb{Q}_{\gamma}$ and $s \leq q \uparrow \beta=\bar{q} \uparrow \beta$. By recursion hypothesis (2)(c) applied to $\mathbb{Q}_{\gamma}, s \cup(\bar{q} \uparrow[\beta, \gamma))$ is in $\mathbb{Q}_{\gamma}$. However, $s \cup(\bar{q} \uparrow[\beta, \gamma))$ is equal to $t\lceil\gamma$.

Now assume $\alpha$ is a successor ordinal. Let $\alpha=\alpha_{0}+1$. First consider the case when $\beta=\alpha_{0}$. If $\alpha_{0}$ is not in $\operatorname{dom}(q)$, then $t=s$, which is in $\mathbb{Q}_{\alpha}$ by (b). Suppose $\alpha_{0}$ is in $\operatorname{dom}(q)$. Then $t=s \cup\left\{\left(\alpha_{0}, q\left(\alpha_{0}\right)\right)\right\}$. Therefore, $t\left\lceil\alpha_{0}=s\right.$, which is in $\mathbb{Q}_{\alpha_{0}}$. By the definition of $\mathbb{Q}_{\alpha}, q\left\lceil\alpha_{0} \Vdash q\left(\alpha_{0}\right) \in \mathbb{Q}\left(\dot{T}\left(\alpha_{0}\right)\right)\right.$. As $s \leq q\left\lceil\alpha_{0}, s=t\left\lceil\alpha_{0}\right.\right.$, and $t\left(\alpha_{0}\right)=q\left(\alpha_{0}\right), t\left\lceil\alpha_{0} \Vdash t\left(\alpha_{0}\right) \in \mathbb{Q}\left(\dot{T}\left(\alpha_{0}\right)\right)\right.$, so $t$ is in $\mathbb{Q}_{\alpha}$. 
Now suppose $\beta<\alpha_{0}$. Then $s \leq q\left\lceil\beta=\left(q\left\lceil\alpha_{0}\right) \uparrow \beta\right.\right.$. By recursion hypothesis (2)(c) applied to $\mathbb{Q}_{\alpha_{0}}$, letting $t^{\prime}=s \cup\left(q\left\lceil\left[\beta, \alpha_{0}\right)\right)\right.$, $t^{\prime}$ is in $\mathbb{Q}_{\alpha_{0}}$ and $t^{\prime} \leq s, q\left\lceil\alpha_{0}\right.$ in $\mathbb{Q}_{\alpha_{0}}$. However, $t^{\prime}=t \uparrow \alpha_{0}$, so $t \uparrow \alpha_{0}$ is in $\mathbb{Q}_{\alpha_{0}}$. If $\alpha_{0}$ is not in $\operatorname{dom}(q)$, then $t^{\prime}=t$ and we are done by (b). Otherwise since $t^{\prime} \leq q\left\lceil\alpha_{0}, t^{\prime}\right.$ forces $q\left(\alpha_{0}\right)=t\left(\alpha_{0}\right)$ is in $\mathbb{Q}\left(\dot{T}\left(\alpha_{0}\right)\right)$. Therefore, $t$ is in $\mathbb{Q}_{\alpha}$.

(d) We show that the inclusion map from $\mathbb{Q}_{\beta}$ to $\mathbb{Q}_{\alpha}$ is a complete embedding. Let $q \leq p$ in $\mathbb{Q}_{\beta}$. Then $\operatorname{dom}(p) \subseteq \operatorname{dom}(q)$, and for all $\nu$ in $\operatorname{dom}(p), q(\nu) \leq_{\mathbb{R}} p(\nu)$. However, this is also the definition of the ordering on $\mathbb{Q}_{\alpha}$, and so $q \leq p$ in $\mathbb{Q}_{\alpha}$. Suppose $p_{1}$ and $p_{2}$ are in $\mathbb{Q}_{\beta}$ and $r \leq p_{1}, p_{2}$ in $\mathbb{Q}_{\alpha}$. By (a), $r \uparrow \beta$ is in $\mathbb{Q}_{\beta}$, and clearly $r \uparrow \beta \leq p_{1}, p_{2}$ in $\mathbb{Q}_{\beta}$. Finally, let $q$ be in $\mathbb{Q}_{\alpha}$. By (c), if $p \leq q \uparrow \beta$ in $\mathbb{Q}_{\beta}$, then $p \cup(q \uparrow[\beta, \alpha))$ is in $\mathbb{Q}_{\alpha}$ and is below $p$ and $q$ in $\mathbb{Q}_{\alpha}$. Thus $p$ and $q$ are compatible in $\mathbb{Q}_{\alpha}$.

\section{Distributivity AND The ChAin CONDition}

Our next goal is to prove that $\mathbb{Q}_{\alpha}$ is $\omega_{1}$-distributive and $\omega_{2}$-c.c., which will complete the verification of the recursion hypotheses. For this purpose, it will be useful to first analyze the iteration in more detail. The proofs in this section are independent of whether $\alpha$ is a successor ordinal or a limit ordinal.

Notation 9.1. Suppose that $p$ is a partial function $p: \alpha \rightarrow \mathbb{R}$ with a countable domain (for example, if $p$ is in $\mathbb{Q}_{\alpha}$ ). For each $\xi$ in $\operatorname{dom}(p)$ ), let $a_{\xi}^{p}$ be $a^{p(\xi)}$, that is, $a_{\xi}^{p}$ is the non-empty countable set $a$ such that $\operatorname{dom}(p(\xi))=a^{2}$.

Occasionally we will consider a partial function $p: \alpha \rightarrow \mathbb{R}$, and write $a_{\xi}^{p}$ and $p(\xi)$, even though we do not know whether $\xi$ is in $\operatorname{dom}(p)$. In this case, $a_{\xi}^{p}$ and $p(\xi)$ both denote the empty set if $\xi$ is not in $\operatorname{dom}(p)$.

The next observation is obvious, but we record it for later reference.

Lemma 9.2. Let $p$ be in $\mathbb{Q}_{\alpha}$. Suppose $\beta$ is in $\operatorname{dom}(p)$ and $x$ is a countable subset of $a_{\beta}^{p}$ which is closed under $p(\beta)$. Then $p\left\lceil\beta \Vdash_{\mathbb{Q}_{\beta}} x \notin \dot{T}(\beta)\right.$.

Proof. By Lemma 8.8(a), $p \uparrow(\beta+1)$ is in $\mathbb{Q}_{\beta+1}$. By the definition of $\mathbb{Q}_{\beta+1}$, $p\left\lceil\beta \Vdash_{\mathbb{Q}_{\beta}} p(\beta) \in \mathbb{Q}(\dot{T}(\beta))\right.$. The conclusion of the lemma now follows by the definition of $\mathbb{Q}(\dot{T}(\beta))$ as given in Definition 7.1.

The next lemma will be useful throughout the remainder of the paper. The proof is straightforward, but we include it for completeness.

Lemma 9.3. Suppose $p$ is a partial function $p: \alpha \rightarrow \mathbb{R}$ with a countable domain. If $p$ is not in $\mathbb{Q}_{\alpha}$, then there exist $\beta, t$, and $x$ such that:

- $\beta$ is in $\operatorname{dom}(p)$,

- $p\left\lceil\beta\right.$ is in $\mathbb{Q}_{\beta}$,

- $t \leq p\left\lceil\beta\right.$ in $\mathbb{Q}_{\beta}$,

- $x$ is a countable subset of $a_{\beta}^{p}$ closed under $p(\beta)$,

- $t \Vdash_{\mathbb{Q}_{\beta}} x \in \dot{T}(\beta)$.

Proof. We assume the statement holds for $\mathbb{Q}_{\nu}$, for all $\nu<\alpha$, and verify that it also holds for $\mathbb{Q}_{\alpha}$. It is clear when $\alpha=0$.

Assume $\alpha$ is a successor ordinal $\alpha_{0}+1$. Let $p$ be a partial function $p: \alpha \rightarrow \mathbb{R}$ with a countable domain, and suppose $p$ is not in $\mathbb{Q}_{\alpha}$. There are two ways this 
could happen. The first possibility is that $p\left\lceil\alpha_{0}\right.$ is not in $\mathbb{Q}_{\alpha_{0}}$. In this case, the conclusion of the lemma follows from the induction hypothesis applied to $p\left\lceil\alpha_{0}\right.$ in $\mathbb{Q}_{\alpha_{0}}$.

The second possibility is that $p\left\lceil\alpha_{0}\right.$ is in $\mathbb{Q}_{\alpha_{0}}, \alpha_{0}$ is in $\operatorname{dom}(p)$, and $p\left\lceil\alpha_{0}\right.$ does not force that $p\left(\alpha_{0}\right)$ is in $\mathbb{Q}\left(\dot{T}\left(\alpha_{0}\right)\right)$. Therefore, there is $t_{0} \leq p\left\lceil\alpha_{0}\right.$ such that $t_{0}$ forces $p\left(\alpha_{0}\right)$ is not in $\mathbb{Q}\left(\dot{T}\left(\alpha_{0}\right)\right)$. Since $p\left(\alpha_{0}\right)$ is in $\mathbb{R}, t_{0}$ forces that there exists a countable set $x \subseteq a^{p\left(\alpha_{0}\right)}$ such that $x$ is closed under $p\left(\alpha_{0}\right)$ and $x$ is in $\dot{T}\left(\alpha_{0}\right)$. By recursion hypothesis (3) applied to $\mathbb{P}_{\alpha_{0}}, \mathbb{P}_{\alpha_{0}}$ is $\omega_{1}$-distributive. Hence $t_{0}$ forces that there is such a set $x$ in the ground model. Hence we can choose $t \leq t_{0}$ and $x$ such that $t$ forces $x$ satisfies the above properties. Then $x$ is a countable subset of $a_{\alpha_{0}}^{p}$ closed under $p\left(\alpha_{0}\right)$ and $t$ forces $x$ is in $\dot{T}\left(\alpha_{0}\right)$.

Now assume $\alpha$ is a limit ordinal. If $p$ is not in $\mathbb{Q}_{\alpha}$, then by the definition of $\mathbb{Q}_{\alpha}$, there is $\gamma<\alpha$ such that $p\left\lceil\gamma\right.$ is not in $\mathbb{Q}_{\gamma}$. The conclusion of the lemma now follows by applying the induction hypothesis to $p\left\lceil\gamma\right.$ in $\mathbb{Q}_{\gamma}$.

Recall that $\Vdash_{\mathbb{Q}_{\beta}} \dot{T}(\beta) \subseteq S_{\omega_{2}} \cap E$. Therefore, if $t \Vdash_{\mathbb{Q}_{\beta}} x \in \dot{T}(\beta)$, as in the conclusion of Lemma 9.3, then $x$ is in $S_{\omega_{2}} \cap E$.

Lemma 9.4. Suppose $p$ and $q$ are conditions in $\mathbb{Q}_{\alpha}$ such that for all $\beta$ in $\operatorname{dom}(p) \cap$ $\operatorname{dom}(q), p(\beta)\left\lceil\left(a_{\beta}^{p} \cap a_{\beta}^{q}\right)^{2}=q(\beta)\left\lceil\left(a_{\beta}^{p} \cap a_{\beta}^{q}\right)^{2}\right.\right.$. Then $p$ and $q$ are compatible. Moreover, there is $r \leq p, q$ such that $\operatorname{dom}(r)=\operatorname{dom}(p) \cup \operatorname{dom}(q)$, for all $\beta$ in $\operatorname{dom}(p) \backslash \operatorname{dom}(q), r(\beta)=p(\beta)$, and for all $\beta$ in $\operatorname{dom}(q) \backslash \operatorname{dom}(p), r(\beta)=q(\beta)$.

In particular, if $p$ and $q$ are conditions in $\mathbb{Q}_{\alpha}$ such that for all $\beta$ in $\operatorname{dom}(p) \cap$ $\operatorname{dom}(q), a_{\beta}^{p} \cap a_{\beta}^{q}$ is empty, then $p$ and $q$ are compatible.

Proof. Choose an ordinal $\zeta$ in $\omega_{1}$ which is not in $a_{\beta}^{p} \cup a_{\beta}^{q}$ for any $\beta$ in $\operatorname{dom}(p) \cap \operatorname{dom}(q)$. Define $r$ as follows. The domain of $r$ is equal to $\operatorname{dom}(p) \cup \operatorname{dom}(q)$. Let $\beta$ be in $\operatorname{dom}(r)$, and we define $r(\beta)$. If $\beta \in \operatorname{dom}(p) \backslash \operatorname{dom}(q)$, let $r(\beta)=p(\beta)$. If $\beta \in \operatorname{dom}(q) \backslash \operatorname{dom}(p)$, let $r(\beta)=q(\beta)$. Suppose $\beta$ is in $\operatorname{dom}(p) \cap \operatorname{dom}(q)$. Define $a_{\beta}^{r}=a_{\beta}^{p} \cup a_{\beta}^{q}$. Let $(i, j)$ be in $\left(a_{\beta}^{r}\right)^{2}$, and we define $r(\beta)(i, j)$. If $(i, j)$ is in $\left(a_{\beta}^{p}\right)^{2}$, let $r(\beta)(i, j)=p(\beta)(i, j)$. If $(i, j)$ is in $\left(a_{\beta}^{q}\right)^{2}$, let $r(\beta)(i, j)=q(\beta)(i, j)$. Note this is well-defined, since if $i$ and $j$ are both in $a_{\beta}^{p} \cap a_{\beta}^{q}$, then $p(\beta)(i, j)=q(\beta)(i, j)$. In any other case, let $r(\beta)(i, j)=\zeta$.

It is easy to see that if $r$ is a condition in $\mathbb{Q}_{\alpha}$, then $r \leq p, q$. Suppose for a contradiction that $r$ is not in $\mathbb{Q}_{\alpha}$. By Lemma 9.3 , fix $\beta, t$, and $x$ such that $\beta$ is in $\operatorname{dom}(r), r \uparrow \beta$ is in $\mathbb{Q}_{\beta}, t \leq r \uparrow \beta$ in $\mathbb{Q}_{\beta}, x$ is a countable subset of $a_{\beta}^{r}$ closed under $r(\beta)$, and $t \Vdash_{\mathbb{Q}_{\beta}} x \in \dot{T}(\beta)$. Note that $r \uparrow \beta$ is below $p \uparrow \beta$ and $q \uparrow \beta$ in $\mathbb{Q}_{\beta}$. Therefore, $t \leq p\lceil\beta, q \uparrow \beta$.

We consider different cases regarding $\beta$, and derive a contradiction in each case. First consider the case that $\beta$ is in $\operatorname{dom}(p) \backslash \operatorname{dom}(q)$. Then by the definition of $r$, $r(\beta)=p(\beta)$. Therefore, $x$ is a countable subset of $a_{\beta}^{p}$ closed under $p(\beta)$. By Lemma 9.2, $p\left\lceil\beta \Vdash_{\mathbb{Q}_{\beta}} x \notin \dot{T}(\beta)\right.$, but this contradicts that $t \leq p\lceil\beta$. A similar argument handles the case when $\beta$ is in $\operatorname{dom}(q) \backslash \operatorname{dom}(p)$.

Now consider the case when $\beta$ is in $\operatorname{dom}(p) \cap \operatorname{dom}(q)$. If $x$ is a subset of $a_{\beta}^{p}$ or $x$ is a subset of $a_{\beta}^{q}$, then the same argument as in the previous paragraph yields a contradiction. Otherwise there is $i$ in $x \backslash a_{\beta}^{p}$ and $j$ in $x \backslash a_{\beta}^{q}$. Then by the definition of $r, r(\beta)(i, j)=\zeta$, which is not in $x$. Therefore, $x$ is not closed under $r(\beta)$, contradicting the choice of $x$. 
Lemma 9.5. Let $z$ be a countable subset of $\alpha$ and let $b$ be a countable subset of $\omega_{2}$. Then there are densely many conditions $t$ in $\mathbb{Q}_{\alpha}$ such that $z \subseteq \operatorname{dom}(t)$, and for all $\beta$ in $z, b \subseteq a_{\beta}^{t}$.

Proof. Let $p$ be a condition in $\mathbb{Q}_{\alpha}$. We will find a condition $t \leq p$ such that $z \subseteq \operatorname{dom}(t)$, and for all $\beta$ in $z, b \subseteq a_{\beta}^{t}$. Fix $\zeta$ in $\omega_{1} \backslash b$. Let $X$ be the set of $\beta$ in $\operatorname{dom}(p) \cap z$ such that $b \subseteq a_{\beta}^{p}$.

Define $s$ in $\mathbb{Q}_{\alpha}$ as follows. The domain of $s$ is equal to $z \backslash X$. Consider $\beta$ in $\operatorname{dom}(s)$. If $\beta$ is in $z \backslash \operatorname{dom}(p)$, let $s(\beta): b^{2} \rightarrow \omega_{1}$ satisfy $s(\beta)(i, j)=\zeta$ for all $(i, j) \in b^{2}$. Suppose $\beta$ is in $z \cap \operatorname{dom}(p)$. Since $\beta$ is not in $X, b \backslash a_{\beta}^{p}$ is non-empty. Define $s(\beta):\left(b \backslash a_{\beta}^{p}\right)^{2} \rightarrow \omega_{1}$ by letting $s(\beta)(i, j)=\zeta$ for all $(i, j)$ in $\left(b \backslash a_{\beta}^{p}\right)^{2}$.

The set $s$ is a partial function $s: \alpha \rightarrow \mathbb{R}$ with a countable domain. Note that $s$ satisfies the property that for all $\beta$ in $\operatorname{dom}(s)$, there are no subsets of $a_{\beta}^{s}$ which are closed under $s(\beta)$. It follows easily from Lemma 9.3 that $s$ is in $\mathbb{Q}_{\alpha}$. Also note that if $\beta$ is in $\operatorname{dom}(s) \cap \operatorname{dom}(p)$, then $a_{\beta}^{s} \cap a_{\beta}^{p}$ is empty. By Lemma 9.4, $s$ and $p$ are compatible. Fix $t \leq s, p$ in $\mathbb{Q}_{\alpha}$. Then clearly $t$ is as desired.

It will be useful to consider a certain kind of condition in $\mathbb{Q}_{\alpha}$, which we call a square condition.

Notation 9.6. A condition $p$ in $\mathbb{Q}_{\alpha}$ is square if there is a set $a$ such that for all $\beta$ in $\operatorname{dom}(p), a_{\beta}^{p}=a$. In this case, we let $a^{p}$ denote the set $a$ as above.

Proposition 9.7. The forcing poset $\mathbb{Q}_{\alpha}$ is $\omega_{1}$-distributive. The set of square conditions is dense in $\mathbb{Q}_{\alpha}$.

Proof. We prove both statements simultaneously. Let $\left\{D_{n}: n<\omega\right\}$ be a family of dense open subsets of $\mathbb{Q}_{\alpha}$, and let $p$ be a condition in $\mathbb{Q}_{\alpha}$. We prove there exists a square condition $q \leq p$ which is in $\bigcap\left\{D_{n}: n<\omega\right\}$.

Fix a regular cardinal $\theta$ much larger than $\mathbb{Q}_{\alpha}$. Recall that we are working in the model $V[H]$, where $H$ is a generic filter on $\mathbb{P}_{\omega_{2}}$ over $V$. We will apply Lemma 4.7 to the forcing poset $\mathbb{P}_{\omega_{2}}=\mathbb{P}\left(\emptyset, 0, \omega_{2}\right)$. By Lemma 4.7, in $V[H]$ there are stationarily many $N$ in $P_{\omega_{1}}(H(\theta))$ such that $S_{\omega_{2}} \cap P\left(N \cap \omega_{2}\right) \subseteq N$. Choose $N$ in $P_{\omega_{1}}(H(\theta))$ such that $N \prec H(\theta)$, the objects $\mathbb{Q}_{\alpha}, p$, and $\left\{D_{n}: n<\omega\right\}$ are in $N$, and $S_{\omega_{2}} \cap P\left(N \cap \omega_{2}\right) \subseteq N$.

Choose an $N$-generic sequence $\left\langle p_{n}: n<\omega\right\rangle$ for $\mathbb{Q}_{\alpha}$, with $p_{0}=p$. By Lemma 9.5 and $N$-genericity, it is easy to see that $\bigcup\left\{\operatorname{dom}\left(p_{n}\right): n<\omega\right\}=N \cap \alpha$, and for each $\beta$ in $N \cap \alpha, \bigcup\left\{a_{\beta}^{p_{n}}: n<\omega\right\}=N \cap \omega_{2}$.

Define $q$ by letting $\operatorname{dom}(q)=\bigcup\left\{\operatorname{dom}\left(p_{n}\right): n<\omega\right\}$, and for each $\beta$ in $\operatorname{dom}(q)$, by letting $q(\beta)$ be the union of the set of functions $\left\{p_{n}(\beta): n<\omega\right\}$. Thus $q$ is a partial function $q: \alpha \rightarrow \mathbb{R}$ with a countable domain. We will prove that $q$ is in $\mathbb{Q}_{\alpha}$. It is then easy to see that $q \leq p_{n}$ for all $n<\omega$, and hence $q$ is in $\bigcap\left\{D_{n}: n<\omega\right\}$. Also, for all $\beta$ in $\operatorname{dom}(q), a_{\beta}^{q}=N \cap \omega_{2}$, so $q$ is a square condition.

Suppose for a contradiction $q$ is not in $\mathbb{Q}_{\alpha}$. By Lemma 9.3, fix $\beta, t$, and $x$ such that $\beta$ is in $\operatorname{dom}(q)=N \cap \alpha, q \uparrow \beta$ is in $\mathbb{Q}_{\beta}, t \leq q \uparrow \beta$ in $\mathbb{Q}_{\beta}, x$ is a countable subset of $a_{\beta}^{q}=N \cap \omega_{2}$ closed under $q(\beta)$, and $t \Vdash_{\mathbb{Q}_{\beta}} x \in \dot{T}(\beta)$. Note that $q \uparrow \beta \leq p_{n} \uparrow \beta$ for all $n<\omega$. Since $t \Vdash_{\mathbb{Q}_{\beta}} x \in \dot{T}(\beta), x$ is in $S_{\omega_{2}}$. Also, $x \subseteq N \cap \omega_{2}$. Therefore, $x$ is in $S_{\omega_{2}} \cap P\left(N \cap \omega_{2}\right)$. By the choice of $N, x$ is in $N$.

Let $D$ be the set of conditions $s$ in $\mathbb{Q}_{\alpha}$ such that $\beta$ is in $\operatorname{dom}(s)$ and $x \subseteq a_{\beta}^{s}$. By Lemma 9.5, $D$ is dense open, and by elementarity, $D$ is in $N$. Fix $n<\omega$ such 
that $p_{n}$ is in $D$. Since $x$ is closed under $q(\beta)$ and $q(\beta) \uparrow\left(a_{\beta}^{p_{n}}\right)^{2}=p_{n}(\beta), x$ is closed under $p_{n}(\beta)$. By Lemma 9.2, $p_{n}\left\lceil\beta \Vdash_{\mathbb{Q}_{\beta}} x \notin \dot{T}(\beta)\right.$. As $t \Vdash_{\mathbb{Q}_{\beta}} x \in \dot{T}(\beta), p_{n}\lceil\beta$ and $t$ are incompatible. But $t \leq q \uparrow \beta \leq p_{n} \uparrow \beta$, so we have a contradiction.

Proposition 9.8. The forcing poset $\mathbb{Q}_{\alpha}$ is $\omega_{2}$-c.c.

Proof. Recall that $\mathrm{CH}$ holds in $V[H]$. We will show that $\mathbb{Q}_{\alpha}$ is $\omega_{2}$-Knaster. Let $\left\langle p_{i}: i<\omega_{2}\right\rangle$ be a sequence of conditions in $\mathbb{Q}_{\alpha}$. Without loss of generality we may assume each $p_{i}$ is a square condition.

Using $\mathrm{CH}$, apply the $\Delta$-System Lemma to the sequence of countable sets $\left\langle\operatorname{dom}\left(p_{i}\right): i<\omega_{2}\right\rangle$ to get an unbounded set $Z_{0} \subseteq \omega_{2}$ and a countable set $b$ such that for all $i<j$ in $Z_{0}, \operatorname{dom}\left(p_{i}\right) \cap \operatorname{dom}\left(p_{j}\right)=b$. Now again apply the $\Delta$-System Lemma to the sequence of countable sets $\left\langle a^{p_{i}}: i \in Z_{0}\right\rangle$ to get an unbounded set $Z_{1} \subseteq Z_{0}$ and a countable set $c$ such that for all $i<j$ in $Z_{1}, a^{p_{i}} \cap a^{p_{j}}=c$.

An easy counting argument using $\mathrm{CH}$ shows there are at most $\omega_{1}$ many possibilities for a sequence $\left\langle p_{i}(\beta)\left\lceil c^{2}: \beta \in b\right\rangle\right.$, for $i$ in $Z_{1}$. Hence, we can fix an unbounded set $Z_{2} \subseteq Z_{1}$ such that for all $i<j$ in $Z_{2}$ and for all $\beta$ in $b, p_{i}(\beta)\left\lceil c^{2}=p_{j}(\beta)\left\lceil c^{2}\right.\right.$.

Consider $i<j$ in $Z_{2}$. Then for all $\beta$ in $\operatorname{dom}\left(p_{i}\right) \cap \operatorname{dom}\left(p_{j}\right)=b, p_{i}(\beta)\left\lceil\left(a^{p_{i}} \cap\right.\right.$ $\left.a^{p_{j}}\right)^{2}=p_{i}(\beta)\left\lceil c^{2}=p_{j}(\beta) \uparrow c^{2}=p_{j}(\beta) \uparrow\left(a^{p_{i}} \cap a^{p_{j}}\right)^{2}\right.$. By Lemma 9.4, $p_{i}$ and $p_{j}$ are compatible.

This completes the verification of the recursion hypotheses.

We conclude this section with several technical lemmas which will be needed later in the paper. Recall that $\mathbb{Q}$ is $\mathbb{Q}_{\omega_{3}}$.

Lemma 9.9. Let $p$ and $q$ be conditions in $\mathbb{Q}$, and assume $\operatorname{dom}(p) \subseteq \operatorname{dom}(q)$, and for all $\beta$ in $\operatorname{dom}(p), a_{\beta}^{p} \subseteq a_{\beta}^{q}$. If $p$ and $q$ are compatible, then $q \leq p$.

Proof. To show $q \leq p$, it suffices to show that whenever $\beta$ is in $\operatorname{dom}(p), q(\beta) \uparrow$ $\left(a_{\beta}^{p}\right)^{2}=p(\beta)$. Fix $r \leq p, q$. Then $q(\beta) \uparrow\left(a_{\beta}^{p}\right)^{2}=r(\beta) \uparrow\left(a_{\beta}^{p}\right)^{2}=p(\beta)$.

Lemma 9.10. Suppose $I$ is a generic filter on $\mathbb{Q}$ over $V[H]$. Let $\left\{p_{n}: n<\omega\right\}$ be a countable subset of $I$. Then there is a condition $r$ in $I$ such that $r \leq p_{n}$ for all $n<\omega$.

Proof. Since $\mathbb{Q}$ is $\omega_{1}$-distributive, the set $\left\{p_{n}: n<\omega\right\}$ is in the ground model $V[H]$. Let $z=\bigcup\left\{\operatorname{dom}\left(p_{n}\right): n<\omega\right\}$ and let $b=\bigcup\left\{a_{\beta}^{p_{n}}: n<\omega, \beta \in \operatorname{dom}\left(p_{n}\right)\right\}$. By Lemma 9.5 , there are densely many conditions $t$ in $\mathbb{Q}$ such that $z \subseteq \operatorname{dom}(t)$, and for all $\beta$ in $z, b \subseteq a_{\beta}^{t}$. Fix such a condition $t$ in $I$. Let $n<\omega$, and we show $t \leq p_{n}$. By the choice of $t, \operatorname{dom}\left(p_{n}\right) \subseteq z \subseteq \operatorname{dom}(t)$, and for all $\beta$ in $\operatorname{dom}\left(p_{n}\right), a_{\beta}^{p_{n}} \subseteq b \subseteq a_{\beta}^{t}$. Also $p_{n}$ and $t$ are both in $I$, so they are compatible. By Lemma $9.9, t \leq p_{n}$.

The next easy lemma is technical and unmotivated, but we will need it in a construction later in the paper.

Lemma 9.11. Suppose $p$ is a square condition in $\mathbb{Q}$. Let $\nu$ be an ordinal in $\omega_{2} \backslash a^{p}$ and let $\zeta$ be an ordinal in $\omega_{1}$ which is different from $\nu$. Let $b$ be a subset of $\operatorname{dom}(p)$. Then there is a condition $t \leq p$ in $\mathbb{Q}$ such that $\operatorname{dom}(t)=\operatorname{dom}(p)$, for all $\beta$ in $b$, $t(\beta)=p(\beta)$, and for all $\beta$ in $\operatorname{dom}(t) \backslash b, \nu \in a_{\beta}^{t}$ and $t(\beta)(\nu, \nu)=\zeta$.

Proof. Define $s$ with domain equal to $\operatorname{dom}(p) \backslash b$ so that for all $\beta$ in $\operatorname{dom}(s), a_{\beta}^{s}=\{\nu\}$ and $s(\beta)(\nu, \nu)=\zeta$. Since for each $\beta$ in $\operatorname{dom}(s)$ there do not exist any subsets of $a_{\beta}^{s}$ which are closed under $s(\beta)$, it follows easily from Lemma 9.3 that $s$ is a condition 
in $\mathbb{Q}$. Now for all $\beta$ in $\operatorname{dom}(p) \cap \operatorname{dom}(s), a_{\beta}^{p} \cap a_{\beta}^{s}$ is empty. By Lemma 9.4, we can choose $t \leq s, p$ such that $\operatorname{dom}(t)=\operatorname{dom}(s) \cup \operatorname{dom}(p)$ (which is equal to $\operatorname{dom}(p)$ ), and for all $\beta$ in $\operatorname{dom}(p) \backslash \operatorname{dom}(s), t(\beta)=p(\beta)$.

Let us verify that $t$ is as desired. We already noted that $\operatorname{dom}(t)=\operatorname{dom}(p)$. If $\beta$ is in $b$, then by the choice of $s, \beta$ is in $\operatorname{dom}(p) \backslash \operatorname{dom}(s)$, so $t(\beta)=p(\beta)$. If $\beta$ is in $\operatorname{dom}(t) \backslash b$, then $\beta$ is in $\operatorname{dom}(p) \backslash b=\operatorname{dom}(s)$. By the choice of $s, \nu$ is in $a_{\beta}^{s}$. Since $t \leq s, \nu$ is in $a_{\beta}^{t}$, and $t(\beta)(\nu, \nu)=s(\beta)(\nu, \nu)=\zeta$.

\section{Preserving the stationarity of $S_{\omega_{2}}$}

In this section we will prove that $\mathbb{Q}$ preserves the stationarity of $S_{\omega_{2}}$ and forces that every stationary subset of $S_{\omega_{2}}$ reflects to an uncountable ordinal in $\omega_{2} \cap \operatorname{cof}(\omega)$.

Proposition 10.1. The forcing poset $\mathbb{Q}$ forces that $S_{\omega_{2}}$ is stationary in $P_{\omega_{1}}\left(\omega_{2}\right)$.

Proof. Recall that $\mathbb{Q}$ is defined in the model $V[H]$, where $H$ is a generic filter on $\mathbb{P}_{\omega_{2}}$ over $V$. Hence, it suffices to show that in $V$ the forcing poset $\mathbb{P}_{\omega_{2}} * \dot{\mathbb{Q}}$ forces $\dot{S}_{\omega_{2}}$ is stationary.

Let $(X, F) * \dot{p}$ be a condition in $\mathbb{P}_{\omega_{2}} * \dot{\mathbb{Q}}$, and suppose $(X, F) * \dot{p}$ forces $\dot{h}:\left[\omega_{2}\right]^{<\omega} \rightarrow$ $\omega_{2}$ is a function. We will find $(Y, K) * \dot{q} \leq(X, F) * \dot{p}$ and a set $z$ such that $(Y, K) * \dot{q}$ forces $z$ is in $\dot{S}_{\omega_{2}}$ and $z$ is closed under $\dot{h}$. Note that $(X, F) * \dot{p}$ forces there are club many ordinals $\nu<\omega_{2}$ which are closed under $\dot{h}$. Choose $\left(X^{\prime}, F^{\prime}\right) * \dot{p}^{\prime} \leq(X, F) * \dot{p}$ and an uncountable ordinal $\nu$ in $\omega_{2} \cap \operatorname{cof}(\omega)$ such that $\left(X^{\prime}, F^{\prime}\right) * \dot{p}^{\prime}$ forces $\nu$ is closed under $\dot{h}$.

For each $\alpha<\omega_{3}, \mathbb{P}_{\omega_{2}} * \dot{\mathbb{Q}}_{\alpha}$ forces that $\dot{T}(\alpha)$ is a subset of $P_{\omega_{1}}\left(\omega_{2}\right)$ which does not reflect to any uncountable ordinal in $\omega_{2}$ with cofinality $\omega$. In particular, $\mathbb{P}_{\omega_{2}} * \dot{\mathbb{Q}}_{\alpha}$ forces that $\dot{T}(\alpha)$ does not reflect to $\nu$. Therefore, choose a $\mathbb{P}_{\omega_{2}} * \dot{\mathbb{Q}}_{\alpha}$-name $\dot{f}_{\alpha}$ for a function $\dot{f}_{\alpha}:[\nu]^{<\omega} \rightarrow \nu$ such that $\mathbb{P}_{\omega_{2}} * \dot{\mathbb{Q}}_{\alpha}$ forces there is no set in $\dot{T}(\alpha) \cap P_{\omega_{1}}(\nu)$ which is closed under $\dot{f}_{\alpha}$.

Fix a regular cardinal $\theta$ much larger than $\mathbb{P}_{\omega_{2}} * \dot{\mathbb{Q}}, \dot{h}$, and $\left\langle\dot{f}_{\alpha}: \alpha<\omega_{3}\right\rangle$. Let $N$ be a countable elementary substructure of $H(\theta)$ which contains as elements the objects $\mathbb{P}_{\omega_{2}} * \dot{\mathbb{Q}},\left(X^{\prime}, F^{\prime}\right) * \dot{p}^{\prime}, \dot{h}, \nu$, and $\left\langle\dot{f}_{\alpha}: \alpha<\omega_{3}\right\rangle$. We attempt to define a condition $(Y, K) * \dot{q}$ below $\left(X^{\prime}, F^{\prime}\right) * \dot{p}^{\prime}$ which is $N$-generic for $\mathbb{P}_{\omega_{2}} * \mathbb{Q}$.

Choose an $N$-generic sequence $\left\langle\left(X_{n}, F_{n}\right) * \dot{p}_{n}: n<\omega\right\rangle$ such that $\left(X_{0}, F_{0}\right) *$ $\dot{p}_{0}=\left(X^{\prime}, F^{\prime}\right) * \dot{p}^{\prime}$. Let $K$ be the infinum of the sequence $\left\langle F_{n}: n<\omega\right\rangle$. Let $Y=\bigcup\left\{X_{n}: n<\omega\right\} \cup\{N \cap \nu\}$. By Proposition 3.8, $(Y, K)$ is a condition in $\mathbb{P}_{\omega_{2}}$ and $(Y, K) \leq\left(X_{n}, F_{n}\right)$ for all $n<\omega$. In particular, $(Y, K)$ is $N$-generic.

Since $N \cap \nu$ is in $Y,(Y, K) \Vdash N \cap \nu \in \dot{S}_{\omega_{2}}$. Using Lemma 3.7, it is easy to see that $\bigcup Y=N \cap \omega_{2}$, and so by Lemma $4.5,(Y, K) \Vdash \dot{S}_{\omega_{2}} \cap P\left(N \cap \omega_{2}\right)=Y$.

Let $H$ be a generic filter on $\mathbb{P}_{\omega_{2}}$ over $V$ which contains the condition $(Y, K)$. Since $(Y, K)$ is $N$-generic, $N[H] \cap V=N$. For each $n<\omega$, let $p_{n}=\dot{p}_{n}^{H}$. Then $\left\langle p_{n}: n<\omega\right\rangle$ is an $N[H]$-generic sequence for $\mathbb{Q}$. By Lemma 9.5 and $N[H]$-genericity, $\bigcup\left\{\operatorname{dom}\left(p_{n}\right): n<\omega\right\}=N[H] \cap \omega_{3}=N \cap \omega_{3}$, and for each $\beta$ in $\bigcup\left\{\operatorname{dom}\left(p_{n}\right): n<\omega\right\}$, $\bigcup\left\{a_{\beta}^{p_{n}}: n<\omega\right\}=N[H] \cap \omega_{2}=N \cap \omega_{2}$.

We define a lower bound $q$ of the sequence $\left\langle p_{n}: n<\omega\right\rangle$. Let the domain of $q$ be equal to $\bigcup\left\{\operatorname{dom}\left(p_{n}\right): n<\omega\right\}$, and for each $\beta$ in $\operatorname{dom}(q)$, let $q(\beta)$ be the union of the set of functions $\left\{p_{n}(\beta): n<\omega\right\}$. Clearly $q$ is a partial function $q: \omega_{3} \rightarrow \mathbb{R}$ with a countable domain. Also, $\operatorname{dom}(q)=N \cap \omega_{3}$, and for each $\beta$ in $\operatorname{dom}(q), a_{\beta}^{q}=N \cap \omega_{2}$. 
We show that $q$ is a condition in $\mathbb{Q}$. It is then clear that $q \leq p_{n}$ for all $n<\omega$. Suppose for a contradiction that $q$ is not a condition. By Lemma 9.3, fix $\beta$, t, and $x$ such that $\beta$ is in $\operatorname{dom}(q), q\left\lceil\beta\right.$ is in $\mathbb{Q}_{\beta}, t \leq q \uparrow \beta$ in $\mathbb{Q}_{\beta}, x$ is a countable subset of $a_{\beta}^{q}$ which is closed under $q(\beta)$, and $t \Vdash_{\mathbb{Q}_{\beta}} x \in \dot{T}(\beta)$. Note that $\beta$ is in $N \cap \omega_{3}$ and $x$ is in $S_{\omega_{2}} \cap P\left(N \cap \omega_{2}\right)=Y$. Clearly $q\left\lceil\beta \leq p_{n} \uparrow \beta\right.$ for all $n<\omega$, so in particular, $q \uparrow \beta$ is $N[H]$-generic for $\mathbb{Q}_{\beta}$.

Now $Y=\bigcup\left\{X_{n}: n<\omega\right\} \cup\{N \cap \nu\}$ and $\bigcup\left\{X_{n}: n<\omega\right\} \subseteq N$, so either $x$ is in $N$ or $x=N \cap \nu$. First suppose $x$ is in $N$. Let $D$ be the dense set of conditions $s$ in $\mathbb{Q}$ such that $\beta \in \operatorname{dom}(s)$ and $x \subseteq a_{\beta}^{s}$. By elementarity, $D$ is in $N$. Fix $n<\omega$ such that $p_{n} \in D$. Then $x \subseteq a_{\beta}^{p_{n}}$. Since $x$ is closed under $q(\beta)$ and $q(\beta) \uparrow\left(a_{\beta}^{p_{n}}\right)^{2}=p_{n}(\beta), x$ is closed under $p_{n}(\beta)$. By Lemma 9.2, $p_{n}\left\lceil\beta \Vdash_{\mathbb{Q}_{\beta}} x \notin \dot{T}(\beta)\right.$. Since $t \Vdash_{\mathbb{Q}_{\beta}} x \in \dot{T}(\beta)$, $p_{n}\left\lceil\beta\right.$ and $t$ are incompatible. However, $t \leq q \uparrow \beta \leq p_{n} \uparrow \beta$, and we have a contradiction.

Now assume $x=N \cap \nu$. Recall that $\mathbb{P}_{\omega_{2}} * \dot{\mathbb{Q}}_{\beta}$ forces that $\dot{f}_{\beta}:[\nu]^{<\omega} \rightarrow \nu$ is a function such that there is no set in $\dot{T}(\beta) \cap P_{\omega_{1}}(\nu)$ which is closed under $\dot{f}_{\beta}$. Since $\beta$ is in $N, \dot{f}_{\beta}$ is in $N$. Let $I_{\beta}$ be a generic filter on $\mathbb{Q}_{\beta}$ over $V[H]$ such that $t$ is in $I_{\beta}$. Since $t \leq q \uparrow \beta$ and $q \uparrow \beta$ is $N[H]$-generic, $N[H]\left[I_{\beta}\right] \cap V[H]=N[H]$. In particular, $N[H]\left[I_{\beta}\right] \cap \nu=N[H] \cap \nu=N \cap \nu$. Let $f_{\beta}=\dot{f}_{\beta}^{H * I_{\beta}}$. Since $\dot{f}_{\beta}$ is in $N$, $f_{\beta}$ is in $N[H]\left[I_{\beta}\right]$. By the elementarity of $N[H]\left[I_{\beta}\right], N[H]\left[I_{\beta}\right] \cap \nu=N \cap \nu$ is closed under $f_{\beta}$. On the other hand, as $t$ is in $I_{\beta}, x=N \cap \nu$ is in $T(\beta)=\dot{T}(\beta)^{I_{\beta}}$. Hence, $x$ is a member of $T(\beta) \cap P_{\omega_{1}}(\nu)$ which is closed under $f_{\beta}$, contradicting the choice of $f_{\beta}$. This completes the proof that $q$ is in $\mathbb{Q}$.

Let $\dot{q}$ be a $\mathbb{P}_{\omega_{2}}$-name such that $(Y, K)$ forces $\dot{q}$ is in $\dot{\mathbb{Q}}$ and $\dot{q} \leq \dot{p}_{n}$ for all $n<\omega$. Then $(Y, K) * \dot{q}$ is $N$-generic for $\mathbb{P}_{\omega_{2}} * \dot{\mathbb{Q}}$, and $(Y, K) * \dot{q} \leq(X, F) * \dot{p}$. We claim that $(Y, K) * \dot{q}$ forces that $N \cap \nu$ is in $\dot{S}_{\omega_{2}}$ and is closed under $\dot{h}$, which completes the proof. Since $(Y, K) * \dot{q} \leq\left(X^{\prime}, F^{\prime}\right) * \dot{p}^{\prime},(Y, K) * \dot{q}$ forces $\nu$ is closed under $\dot{h}$. As $\dot{h}$ is in $N,(Y, K) * \dot{q}$ forces $N[\dot{H} * \dot{I}] \cap \omega_{2}$ is closed under $\dot{h}$, where $\dot{H} * \dot{I}$ is the canonical $\mathbb{P}_{\omega_{2}} * \dot{\mathbb{Q}}$-name for the generic filter. Therefore, $(Y, K) * \dot{q}$ forces $N[\dot{H} * \dot{I}] \cap \nu$ is closed under $\dot{h}$. As $(Y, K) * \dot{q}$ is $N$-generic, $(Y, K) * \dot{q} \Vdash N[\dot{H} * \dot{I}] \cap \nu=N \cap \nu$. However, $N \cap \nu$ is in $Y$, so $(Y, K) * \dot{q}$ forces $N \cap \nu$ is in $\dot{S}_{\omega_{2}}$ and is closed under $\dot{h}$.

Theorem 10.2. The forcing poset $\mathbb{Q}$ forces that $S_{\omega_{2}}$ is a stationary subset of $P_{\omega_{1}}\left(\omega_{2}\right)$ which does not reflect to any ordinal in $\omega_{2}$ with cofinality $\omega_{1}$, and for every stationary set $T \subseteq S_{\omega_{2}}$, there is an uncountable ordinal $\nu$ in $\omega_{2}$ with cofinality $\omega$ such that $T$ reflects to $\nu$.

Proof. Let $p$ be a condition in $\mathbb{Q}$ and suppose $p$ forces $\dot{U}$ is a subset of $S_{\omega_{2}}$ which does not reflect to any uncountable ordinal in $\omega_{2}$ with cofinality $\omega$. We will prove that $p$ forces $\dot{U}$ is non-stationary. Since $\mathbb{Q}$ is $\omega_{1}$-distributive, it is easy to see that the set $E$ from Notation 8.4 is still club in any generic extension by $\mathbb{Q}$. It suffices to show that $p$ forces $\dot{U} \cap E$ is non-stationary.

Fix a nice $\mathbb{Q}$-name $\dot{T}$ for a subset of $P_{\omega_{1}}\left(\omega_{2}\right)$ such that $p \Vdash_{\mathbb{Q}} \dot{T}=\dot{U} \cap E$, and $\mathbb{Q}$ forces that if $p$ is not in the generic filter, then $\dot{T}$ is the empty set. For some sequence of antichains $\left\langle A_{x}: x \in P_{\omega_{1}}\left(\omega_{2}\right)\right\rangle$ of $\mathbb{Q}, \dot{T}$ is equal to $\bigcup\left\{A_{x} \times\{\check{x}\}: x \in P_{\omega_{1}}\left(\omega_{2}\right)\right\}$. As $\mathbb{Q}$ is $\omega_{2}$-c.c., each $A_{x}$ has cardinality at most $\omega_{1}$. Since $P_{\omega_{1}}\left(\omega_{2}\right)$ has cardinality $\omega_{2}$ and $\mathbb{Q}=\bigcup\left\{\mathbb{Q}_{\alpha}: \alpha<\omega_{3}\right\}$, we can fix $\beta<\omega_{3}$ such that for all $x$ in $P_{\omega_{1}}\left(\omega_{2}\right)$, $A_{x} \subseteq \mathbb{Q}_{\beta}$. Then $\dot{T}$ is a nice $\mathbb{Q}_{\beta}$-name. 
For each $\nu$ in $\omega_{2}$ with cofinality $\omega, \Vdash_{\mathbb{Q}} \dot{T}$ does not reflect to $\nu$. Hence, we can choose a nice $\mathbb{Q}$-name $\dot{C}_{\nu}$ for a club subset of $P_{\omega_{1}}(\nu)$ which is disjoint from $\dot{T}$. As above, choose $\xi<\omega_{3}$ larger than $\beta$ such that for all such $\nu, \dot{C}_{\nu}$ is a $\mathbb{Q}_{\xi}$-name. Now it is easy to see that $\mathbb{Q}_{\xi}$ forces that $\dot{C}_{\nu} \cap \dot{T}$ is empty, for all uncountable $\nu$ in $\omega_{2}$ with cofinality $\omega$.

In summary, $\dot{T}$ is a nice $\mathbb{Q}_{\xi}$-name for a subset of $P_{\omega_{1}}\left(\omega_{2}\right)$ such that $\mathbb{Q}_{\xi}$ forces that $\dot{T} \subseteq S_{\omega_{2}} \cap E$, and for all uncountable $\nu$ in $\omega_{2}$ with cofinality $\omega, \dot{T}$ does not reflect to $\nu$. By recursion hypothesis (4), there is $i<\omega_{3}$ such that $\dot{T}=\dot{T}_{i}^{\xi}$.

Let $\gamma<\omega_{3}$ be an ordinal such that $f(\gamma)=(\xi, i)$. Then $\dot{T}(\gamma)=\dot{T}_{i}^{\xi}=\dot{T}$. By Lemma 8.7, $\mathbb{Q}_{\gamma+1}$ adds a club subset of $P_{\omega_{1}}\left(\omega_{2}\right)$ which is disjoint from $\dot{T}$. Since the inclusion map $\mathbb{Q}_{\gamma+1} \rightarrow \mathbb{Q}$ is a complete embedding, $\mathbb{Q}$ adds a club which is disjoint from $\dot{T}$. As $p \Vdash \dot{T}=\dot{U} \cap E, p$ forces $\dot{U} \cap E$ is non-stationary.

\section{Extending an elementary embedding, Part 2}

For the remainder of the section fix a cardinal $\kappa$ which is $\kappa^{+}$-supercompact, and assume $2^{\kappa}=\kappa^{+}$. Let $j: V \rightarrow M$ be an elementary embedding with critical point $\kappa$ such that $j(\kappa)>\kappa^{+}$and $M^{\kappa^{+}} \subseteq M$. In Section 6 we showed how to extend the elementary embedding $j$ after forcing with $\operatorname{CoLL}\left(\omega_{1},<\kappa\right) * \dot{\mathbb{P}}_{\kappa}$. Specifically, let $\bar{G} * \bar{H}$ be a generic filter on $j\left(\operatorname{Colv}\left(\omega_{1},<\kappa\right) * \dot{\mathbb{P}}_{\kappa}\right)$ over $V$. Let $G=\bar{G} \cap \operatorname{Colv}\left(\omega_{1},<\kappa\right)$ and $H=\bar{H} \cap \mathbb{P}_{\kappa}$. Then $G * H$ is a generic filter on $\operatorname{CoLL}\left(\omega_{1},<\kappa\right) * \dot{\mathbb{P}}_{\kappa}$ over $V$, and in $V[\bar{G} * \bar{H}]$ we can extend $j$ to $j: V[G * H] \rightarrow M[\bar{G} * \bar{H}]$ such that $j(G * H)=\bar{G} * \bar{H}$.

In this section we will prove that in $M[\bar{G} * \bar{H}]$ there is a projection mapping $\pi: j(\mathbb{Q}) \rightarrow \mathbb{Q}$. As a consequence, if $\bar{I}$ is a generic filter for $j(\mathbb{Q})$ over $V[\bar{G} * \bar{H}]$, then in $V[\bar{G} * \bar{H} * \bar{I}]$ we can extend $j$ to $j: V[G * H * I] \rightarrow M[\bar{G} * \bar{H} * \bar{I}]$ such that $j(G * H * I)=\bar{G} * \bar{H} * \bar{I}$, where $I$ is the filter on $\mathbb{Q}$ generated by $\pi[\bar{I}]$.

As in Section 6, we write $\kappa^{+}$to indicate the ordinal which is the successor of $\kappa$ in $V$, even when working in a model in which this ordinal is no longer a cardinal. In the models $M[G * H]$ and $V[G * H], \kappa$ is equal to $\omega_{2}$ and $\kappa^{+}$is equal to $\omega_{3}$. However, in the model $M[\bar{G} * \bar{H}], j(\kappa)$ is equal to $\omega_{2}$, and $\kappa$ and $\kappa^{+}$are both ordinals of cofinality $\omega_{1}$ strictly between $\omega_{1}$ and $\omega_{2}$.

By Theorem 6.1(1), $M[G * H]^{\kappa^{+}} \cap V[G * H] \subseteq M[G * H]$. It easily follows from this fact and from Recursion hypotheses 8.5 (1) and (4) that the sequences $\left\langle\mathbb{Q}_{\alpha}: \alpha \leq \kappa^{+}\right\rangle$and $\left\langle\dot{T}(\alpha): \alpha<\kappa^{+}\right\rangle$are in $M[G * H]$.

Recall that in $V[G * H], \mathbb{R}$ is the poset consisting of all non-empty functions $s:\left(a^{s}\right)^{2} \rightarrow \omega_{1}$, where $a^{s}$ is a countable subset of $\omega_{2}$, ordered by extension of functions.

In the next lemma and in what follows, $\mathbb{R}$ means $(\mathbb{R})^{V[G * H]}$.

Lemma 11.1. In $M[\bar{G} * \bar{H}], \mathbb{R}=\left\{s \in j(\mathbb{R}): a^{s} \subseteq \kappa\right\}$. Hence, if $t$ is in $j(\mathbb{R})$, then $t \uparrow \kappa^{2}$ is in $\mathbb{R}$.

Proof. Let $s$ be in $\mathbb{R}$. Since $s$ is a countable subset of $\kappa^{2} \times \omega_{1}, j(s)=s$. Therefore, by the elementarity of $j, s$ is in $j(\mathbb{R})$, and obviously $a^{s} \subseteq \kappa$. On the other hand, suppose $s$ is in $j(\mathbb{R})$ and $a^{s} \subseteq \kappa$. Since $s$ is a countable subset of $\kappa^{2} \times \omega_{1}$ and $M[\bar{G} * \bar{H}]$ is a generic extension of $M$ by an $\omega_{1}$-distributive forcing poset, $s$ is in $M$. Hence, $s$ is in $V$, and thus $s$ is in $V[G * H]$. As above, $j(s)=s$. Since $j(s)$ is in $j(\mathbb{R}), s$ is in $\mathbb{R}$. 
We will define for each $\alpha \leq \kappa^{+}$a map $\pi_{\alpha}: j\left(\mathbb{Q}_{\alpha}\right) \rightarrow \mathbb{Q}_{\alpha}$ in $M[\bar{G} * \bar{H}]$. Fix $\alpha \leq \kappa^{+}$, and consider a condition $q$ in $j\left(\mathbb{Q}_{\alpha}\right)$. Then $q$ is a partial function $q: j(\alpha) \rightarrow j(\mathbb{R})$ with a countable domain. Therefore, $\operatorname{dom}(q) \cap j[\alpha]$ is a countable subset of $j[\alpha]$, and the set $j^{-1}(\operatorname{dom}(q) \cap j[\alpha])$ is a countable subset of $\alpha$. Since $M[\bar{G} * \bar{H}]$ is a generic extension of $M$ by an $\omega_{1}$-distributive forcing poset, $j^{-1}(\operatorname{dom}(q) \cap j[\alpha])$ is in $M$ and hence is in $V[G * H]$.

For each ordinal $\gamma$ in $j^{-1}(\operatorname{dom}(q) \cap j[\alpha]), q(j(\gamma))$ is in $j(\mathbb{R})$, so $q(j(\gamma)) \uparrow \kappa^{2}$ is in $\mathbb{R}$ by Lemma 11.1. Define $\pi_{\alpha}(q)$ as the function with domain equal to $j^{-1}(\operatorname{dom}(q) \cap j[\alpha])$ such that for all $\gamma$ in this domain, $\pi_{\alpha}(q)(\gamma)=q(j(\gamma)) \uparrow \kappa^{2}$. Clearly $\pi_{\alpha}(q)$ is a partial function $\pi_{\alpha}(q): \alpha \rightarrow \mathbb{R}$ with a countable domain, and again by distributivity, $\pi_{\alpha}(q)$ is in $V[G * H]$. It will take some work to prove that $\pi_{\alpha}(q)$ is in $\mathbb{Q}_{\alpha}$.

We record our definition of $\pi_{\alpha}$ for easy reference.

Definition 11.2. Let $\alpha \leq \kappa^{+}$. In $M[\bar{G} * \bar{H}]$ define a map $\pi_{\alpha}$ with domain $j\left(\mathbb{Q}_{\alpha}\right)$ as follows. Given $q$ in $j\left(\mathbb{Q}_{\alpha}\right)$, let the domain of $\pi_{\alpha}(q)$ be equal to $j^{-1}(\operatorname{dom}(q) \cap j[\alpha])$, and for each $\gamma$ in this domain, let $\pi_{\alpha}(q)(\gamma)=q(j(\gamma)) \uparrow \kappa^{2}$.

The next lemma is immediate from the definition.

Lemma 11.3. Let $\alpha \leq \kappa^{+}$. For all $q$ in $j\left(\mathbb{Q}_{\alpha}\right), j\left[\operatorname{dom}\left(\pi_{\alpha}(q)\right)\right]=\operatorname{dom}(q) \cap j[\alpha]$, and for all $\gamma$ in the domain of $\pi_{\alpha}(q), a_{\gamma}^{\pi_{\alpha}(q)}=a_{j(\gamma)}^{q} \cap \kappa$.

Lemma 11.4. Let $\alpha_{1} \leq \alpha_{2} \leq \kappa^{+}$and let $q$ be in $j\left(\mathbb{Q}_{\alpha_{2}}\right)$. Then $\pi_{\alpha_{1}}\left(q \uparrow j\left(\alpha_{1}\right)\right)=$ $\pi_{\alpha_{2}}(q) \uparrow \alpha_{1}$.

Proof. The domain of $\pi_{\alpha_{1}}\left(q \uparrow j\left(\alpha_{1}\right)\right)$ is equal to $j^{-1}\left(\operatorname{dom}\left(q \uparrow j\left(\alpha_{1}\right)\right) \cap j\left[\alpha_{1}\right]\right)$, whereas the domain of $\pi_{\alpha_{2}}(q) \uparrow \alpha_{1}$ is $j^{-1}\left(\operatorname{dom}(q) \cap j\left[\alpha_{2}\right]\right) \cap \alpha_{1}$, and it is easy to see these sets are equal. Let $\gamma$ be in the domain of $\pi_{\alpha_{1}}\left(q\left\lceil j\left(\alpha_{1}\right)\right)\right.$. Then $\pi_{\alpha_{1}}\left(q\left\lceil j\left(\alpha_{1}\right)\right)(\gamma)\right.$ is equal to $\left(q\left\lceil j\left(\alpha_{1}\right)\right)(j(\gamma)) \uparrow \kappa^{2}=q(j(\gamma)) \uparrow \kappa^{2}=\pi_{\alpha_{2}}(q)(\gamma)=\left(\pi_{\alpha_{2}}(q) \uparrow \alpha_{1}\right)(\gamma)\right.$.

Lemma 11.5. Let $\alpha \leq \kappa^{+}$and let $q$ be in $j\left(\mathbb{Q}_{\alpha}\right)$. Supposet is in $\mathbb{Q}_{\alpha}, \operatorname{dom}\left(\pi_{\alpha}(q)\right) \subseteq$ $\operatorname{dom}(t)$, and for all $\gamma$ in $\operatorname{dom}\left(\pi_{\alpha}(q)\right), t(\gamma) \leq_{\mathbb{R}} \pi_{\alpha}(q)(\gamma)$. Then $j(t)$ and $q$ are compatible in $j\left(\mathbb{Q}_{\alpha}\right)$. In particular, if $\pi_{\alpha}(q)$ is in $\mathbb{Q}_{\alpha}$ and $t \leq \pi_{\alpha}(q)$ in $\mathbb{Q}_{\alpha}$, then $j(t)$ and $q$ are compatible in $j\left(\mathbb{Q}_{\alpha}\right)$.

Proof. To show that $j(t)$ and $q$ are compatible in $j\left(\mathbb{Q}_{\alpha}\right)$, by Lemma 9.4 it suffices to show that for all $\beta$ in $\operatorname{dom}(j(t)) \cap \operatorname{dom}(q)$,

$$
j(t)(\beta) \uparrow\left(a_{\beta}^{j(t)} \cap a_{\beta}^{q}\right)^{2}=q(\beta) \uparrow\left(a_{\beta}^{j(t)} \cap a_{\beta}^{q}\right)^{2} .
$$

First we claim that

$$
\operatorname{dom}(j(t)) \cap \operatorname{dom}(q)=\operatorname{dom}(q) \cap j[\alpha] .
$$

The domain of $t$ is a countable subset of $\alpha$, so $\operatorname{dom}(j(t))=j(\operatorname{dom}(t))=j[\operatorname{dom}(t)]$, which is a subset of $j[\alpha]$. Therefore, $\operatorname{dom}(j(t)) \cap \operatorname{dom}(q) \subseteq \operatorname{dom}(q) \cap j[\alpha]$. Suppose on the other hand $\beta$ is in $\operatorname{dom}(q) \cap j[\alpha]$. Recall that $\operatorname{dom}\left(\pi_{\alpha}(q)\right)=j^{-1}(\operatorname{dom}(q) \cap j[\alpha])$. Therefore, letting $\gamma=j^{-1}(\beta), \gamma$ is in the domain of $\pi_{\alpha}(q)$. But $\operatorname{dom}\left(\pi_{\alpha}(q)\right) \subseteq$ $\operatorname{dom}(t)$ by assumption, so $\gamma$ is in the domain of $t$. Hence $j(\gamma)=\beta$ is in $\operatorname{dom}(j(t)) \cap$ $\operatorname{dom}(q)$.

Next we claim that for all $\beta$ in the domain of $j(t)$, letting $\gamma=j^{-1}(\beta)$,

$$
a_{\beta}^{j(t)}=a_{\gamma}^{t}
$$


and

$$
j(t)(\beta)=t(\gamma) .
$$

Let $\beta=j(\gamma)$ be in the domain of $j(t)$. Then $t(\gamma):\left(a_{\gamma}^{t}\right)^{2} \rightarrow \omega_{1}$, where $a_{\gamma}^{t}$ is a countable subset of $\kappa$, so $a_{\beta}^{j(t)}=j\left(a_{\gamma}^{t}\right)=a_{\gamma}^{t}$, proving the first claim. Now for any pair $(i, k)$ in $\left(a_{\beta}^{j(t)}\right)^{2}, j(t)(\beta)(i, k)=j(t)(j(\gamma))(j(i), j(k))=j(t(\gamma)(i, k))=$ $t(\gamma)(i, k)$, so $j(t)(\beta)=t(\gamma)$.

Now we prove that for all $\beta$ in $\operatorname{dom}(j(t)) \cap \operatorname{dom}(q)$, letting $\gamma=j^{-1}(\beta)$,

$$
a_{\beta}^{j(t)} \cap a_{\beta}^{q}=a_{\gamma}^{\pi_{\alpha}(q)} .
$$

This makes sense since if $\beta$ is in $\operatorname{dom}(j(t)) \cap \operatorname{dom}(q)$, then $\beta$ is in $\operatorname{dom}(q) \cap j[\alpha]$, so $\gamma$ is in $j^{-1}(\operatorname{dom}(q) \cap j[\alpha])=\operatorname{dom}\left(\pi_{\alpha}(q)\right)$. Since $a_{\beta}^{j(t)}=a_{\gamma}^{t}$, which is a subset of $\kappa$, $a_{\beta}^{j(t)} \cap a_{\beta}^{q} \subseteq a_{\beta}^{q} \cap \kappa=a_{\gamma}^{\pi_{\alpha}(q)}$. On the other hand, $t(\gamma) \leq_{\mathbb{R}} \pi_{\alpha}(q)(\gamma)$ by assumption. In particular, $a_{\gamma}^{\pi_{\alpha}(q)} \subseteq a_{\gamma}^{t}=a_{\beta}^{j(t)}$. Also $a_{\gamma}^{\pi_{\alpha}(q)}=a_{\beta}^{q} \cap \kappa \subseteq a_{\beta}^{q}$, so $a_{\gamma}^{\pi_{\alpha}(q)} \subseteq a_{\beta}^{j(t)} \cap a_{\beta}^{q}$.

We finish the proof. Let $\beta$ be in $\operatorname{dom}(j(t)) \cap \operatorname{dom}(q)$. Then $\beta$ is in $\operatorname{dom}(q) \cap j[\alpha]$. Let $\gamma=j^{-1}(\beta)$. Then $a_{\beta}^{j(t)} \cap a_{\beta}^{q}=a_{\gamma}^{\pi_{\alpha}(q)}$ and $j(t)(\beta)=t(\gamma)$. Since $t(\gamma) \leq_{\mathbb{R}}$ $\pi_{\alpha}(q)(\gamma), j(t)(\beta) \uparrow\left(a_{\beta}^{j(t)} \cap a_{\beta}^{q}\right)^{2}=t(\gamma) \uparrow\left(a_{\gamma}^{\pi_{\alpha}(q)}\right)^{2}=\pi_{\alpha}(q)(\gamma)=q(\beta) \uparrow\left(a_{\gamma}^{\pi_{\alpha}(q)}\right)^{2}=$ $q(\beta) \uparrow\left(a_{\beta}^{j(t)} \cap a_{\beta}^{q}\right)^{2}$.

Lemma 11.6. Let $\alpha \leq \kappa^{+}$and let $t$ be in $\mathbb{Q}_{\alpha}$. Suppose $u \leq j(t)$ in $j\left(\mathbb{Q}_{\alpha}\right)$. Then $\operatorname{dom}(t) \subseteq \operatorname{dom}\left(\pi_{\alpha}(u)\right)$, and for all $\gamma$ in $\operatorname{dom}(t), \pi_{\alpha}(u)(\gamma) \leq_{\mathbb{R}} t(\gamma)$. In particular, if $\pi_{\alpha}(u)$ is in $\mathbb{Q}_{\alpha}$, then $\pi_{\alpha}(u) \leq t$ in $\mathbb{Q}_{\alpha}$.

Proof. Since $\operatorname{dom}(t)$ is a countable subset of $\alpha, \operatorname{dom}(j(t))=j(\operatorname{dom}(t))=j[\operatorname{dom}(t)]$. As $u \leq j(t)$ in $j\left(\mathbb{Q}_{\alpha}\right), j[\operatorname{dom}(t)] \subseteq \operatorname{dom}(u)$. Therefore, if $\gamma$ is in $\operatorname{dom}(t), j(\gamma)$ is in $\operatorname{dom}(u) \cap j[\alpha]$, so $\gamma$ is in $j^{-1}(\operatorname{dom}(u) \cap j[\alpha])=\operatorname{dom}\left(\pi_{\alpha}(u)\right)$. This shows $\operatorname{dom}(t) \subseteq \operatorname{dom}\left(\pi_{\alpha}(u)\right)$.

Now let $\gamma$ be in $\operatorname{dom}(t)$, and we show $\pi_{\alpha}(u)(\gamma) \leq_{\mathbb{R}} t(\gamma)$. Note that $j(t)(j(\gamma))=$ $j(t(\gamma))=t(\gamma)$, because $t(\gamma)$ is a countable subset of $\kappa^{2} \times \omega_{1}$. Since $u \leq j(t)$ in $j\left(\mathbb{Q}_{\alpha}\right), u(j(\gamma)) \leq_{j(\mathbb{R})} j(t)(j(\gamma))=t(\gamma)$. In other words, $u(j(\gamma))$ extends $t(\gamma)$ as a function. But $a_{\gamma}^{t}$ is a subset of $\kappa$, so $u(j(\gamma)) \uparrow \kappa^{2}=\pi_{\alpha}(u)(\gamma)$ extends $t(\gamma)$ as a function. Therefore, $\pi_{\alpha}(u)(\gamma) \leq_{\mathbb{R}} t(\gamma)$.

Now we are ready to show $\pi_{\alpha}: j\left(\mathbb{Q}_{\alpha}\right) \rightarrow \mathbb{Q}_{\alpha}$ is a projection mapping.

Proposition 11.7. For all $\alpha \leq \kappa^{+}, \pi_{\alpha}$ is a projection mapping $\pi_{\alpha}: j\left(\mathbb{Q}_{\alpha}\right) \rightarrow \mathbb{Q}_{\alpha}$. Moreover, if $t \leq \pi_{\alpha}(q)$ in $\mathbb{Q}_{\alpha}$, then $j(t)$ and $q$ are compatible in $j\left(\mathbb{Q}_{\alpha}\right)$.

Proof. The proof is by induction on $\alpha \leq \kappa^{+}$. Let $\alpha \leq \kappa^{+}$be given, and assume for all $\beta<\alpha$ that $\pi_{\beta}$ satisfies the statement of the proposition.

First we prove that if $q$ is in $j\left(\mathbb{Q}_{\alpha}\right)$, then $\pi_{\alpha}(q)$ is in $\mathbb{Q}_{\alpha}$. We observed above that $\pi_{\alpha}(q)$ is in $V[G * H]$ and $\pi_{\alpha}(q)$ is a partial function $\pi_{\alpha}(q): \alpha \rightarrow \mathbb{R}$ with a countable domain. Hence, if $\pi_{\alpha}(q)$ is not in $\mathbb{Q}_{\alpha}$, then by Lemma 9.3 , we can fix $\beta$, $u$, and $x$ such that $\beta$ is in $\operatorname{dom}\left(\pi_{\alpha}(q)\right), \pi_{\alpha}(q)\left\lceil\beta\right.$ is in $\mathbb{Q}_{\beta}, u \leq \pi_{\alpha}(q)\left\lceil\beta\right.$ in $\mathbb{Q}_{\beta}, x$ is a countable subset of $a_{\beta}^{\pi_{\alpha}(q)}$ which is closed under $\pi_{\alpha}(q)(\beta)$, and $u \Vdash_{\mathbb{Q}_{\beta}} x \in \dot{T}(\beta)$.

The set $x$ is a countable subset of $\kappa$, so $j(x)=x$. By Lemma 11.4, $\pi_{\beta}(q \uparrow$ $j(\beta))=\pi_{\alpha}(q)\left\lceil\beta\right.$. Therefore, $u \leq \pi_{\beta}\left(q\lceil j(\beta))\right.$ in $\mathbb{Q}_{\beta}$. Applying Lemma 11.5 to $u$ and $q \uparrow j(\beta)$, we get that $j(u)$ and $q \uparrow j(\beta)$ are compatible in $j\left(\mathbb{Q}_{\beta}\right)$. Fix $s \leq j(u), q\left\lceil j(\beta)\right.$ in $j\left(\mathbb{Q}_{\beta}\right)$. 
Recall that $u \Vdash_{\mathbb{Q}_{\beta}} x \in \dot{T}(\beta)$. Since $j(x)=x$ and $s \leq j(u)$, by the elementarity of $j, s \Vdash_{j\left(\mathbb{Q}_{\beta}\right)} x \in j(\dot{T}(\beta))$. The set $x$ is a subset of $a_{\beta}^{\pi_{\alpha}(q)}=a_{j(\beta)}^{q} \cap \kappa$, and $x$ is closed under $\pi_{\alpha}(q)(\beta)=q(j(\beta)) \uparrow \kappa^{2}$. Therefore, $x$ is a countable subset of $a_{j(\beta)}^{q}$ which is closed under $q(j(\beta))$. By Lemma $9.2, q\left\lceil j(\beta) \Vdash_{j\left(\mathbb{Q}_{\beta}\right)} x \notin j(\dot{T}(\beta))\right.$. However, $s \leq q\left\lceil j(\beta)\right.$ and $s \Vdash_{j\left(\mathbb{Q}_{\beta}\right)} x \in j(\dot{T}(\beta))$. This is a contradiction. Therefore, indeed $\pi_{\alpha}(q)$ is in $\mathbb{Q}_{\alpha}$.

Now we prove that $\pi_{\alpha}: j\left(\mathbb{Q}_{\alpha}\right) \rightarrow \mathbb{Q}_{\alpha}$ is a projection mapping. Clearly $\pi_{\alpha}$ sends the empty condition in $j\left(\mathbb{Q}_{\alpha}\right)$ to the empty condition in $\mathbb{Q}_{\alpha}$. Suppose $r \leq q$ in $j\left(\mathbb{Q}_{\alpha}\right)$, and we show $\pi_{\alpha}(r) \leq \pi_{\alpha}(q)$ in $\mathbb{Q}_{\alpha}$. Since $\operatorname{dom}(q) \subseteq \operatorname{dom}(r), \operatorname{dom}\left(\pi_{\alpha}(q)\right)=$ $j^{-1}(\operatorname{dom}(q) \cap j[\alpha]) \subseteq j^{-1}(\operatorname{dom}(r) \cap j[\alpha])=\operatorname{dom}\left(\pi_{\alpha}(r)\right)$. Let $\gamma$ be in $\operatorname{dom}\left(\pi_{\alpha}(q)\right)$. Then $j(\gamma)$ is in $\operatorname{dom}(q)$, and since $r \leq q, r(j(\gamma))$ extends $q(j(\gamma))$ as a function. It follows that $r(j(\gamma)) \uparrow \kappa^{2}$ extends $q(j(\gamma)) \uparrow \kappa^{2}$ as a function, that is, $\pi_{\alpha}(r)(\gamma) \leq_{\mathbb{R}}$ $\pi_{\alpha}(q)(\gamma)$. Finally, let $q$ be in $j\left(\mathbb{Q}_{\alpha}\right)$, and suppose $v \leq \pi_{\alpha}(q)$ in $\mathbb{Q}_{\alpha}$. We show there is $u \leq q$ in $j\left(\mathbb{Q}_{\alpha}\right)$ such that $\pi_{\alpha}(u) \leq v$ in $\mathbb{Q}_{\alpha}$. By Lemma 11.5, $j(v)$ and $q$ are compatible in $j\left(\mathbb{Q}_{\alpha}\right)$, so fix $u \leq j(v), q$. Then by Lemma 11.6, $\pi_{\alpha}(u) \leq v$ in $\mathbb{Q}_{\alpha}$.

We have established that $\pi_{\alpha}: j\left(\mathbb{Q}_{\alpha}\right) \rightarrow \mathbb{Q}_{\alpha}$ is a projection mapping. If $t \leq \pi_{\alpha}(q)$ in $\mathbb{Q}_{\alpha}$, then $j(t)$ and $q$ are compatible in $j\left(\mathbb{Q}_{\alpha}\right)$ by Lemma 11.5 .

Let $\pi: j(\mathbb{Q}) \rightarrow \mathbb{Q}$ be the map $\pi_{\kappa^{+}}$.

We now turn to showing that we can extend the elementary embedding $j$ after forcing with $\mathbb{Q}$. First we need several easy technical lemmas.

Lemma 11.8. Let $\alpha \leq \kappa^{+}$. If $q$ is in $j\left(\mathbb{Q}_{\alpha}\right)$ and $\pi_{\alpha}(q) \leq p$ in $\mathbb{Q}_{\alpha}$, then $q \leq j(p)$ in $j\left(\mathbb{Q}_{\alpha}\right)$.

Proof. The domain of $j(p)$ is equal to $j(\operatorname{dom}(p))=j[\operatorname{dom}(p)]$, and for all $j(\gamma)$ in $\operatorname{dom}(j(p)), j(p)(j(\gamma))=j(p(\gamma))=p(\gamma)$, since $p(\gamma)$ is a countable subset of $\kappa^{2} \times \omega_{1}$.

First we show $\operatorname{dom}(j(p)) \subseteq \operatorname{dom}(q)$. Let $\beta$ be in $\operatorname{dom}(j(p))$. Then $\beta=j(\gamma)$ for some $\gamma$ in $\operatorname{dom}(p)$. Since $\pi_{\alpha}(q) \leq p, \gamma$ is in $\operatorname{dom}\left(\pi_{\alpha}(q)\right)=j^{-1}(\operatorname{dom}(q) \cap j[\alpha])$. Hence, $j(\gamma)=\beta$ is in $\operatorname{dom}(q)$.

Now let $\beta=j(\gamma)$ be in $\operatorname{dom}(j(p))$. Then $q(\beta)\left\lceil\kappa^{2}=\pi_{\alpha}(q)(\gamma) \leq_{\mathbb{R}} p(\gamma)=\right.$ $j(p)(\beta)$, that is, $q(\beta) \uparrow \kappa^{2}$ extends $j(p)(\beta)$ as a function. Therefore, $q(\beta)$ extends $j(p)(\beta)$ as a function, that is, $q(\beta) \leq_{j(\mathbb{R})} j(p)(\beta)$.

Lemma 11.9. Let $\alpha \leq \kappa^{+}$. If $p$ is in $\mathbb{Q}_{\alpha}$, then $\pi_{\alpha}(j(p))=p$.

Proof. The domain of $\pi_{\alpha}(j(p))$ is equal to $j^{-1}(\operatorname{dom}(j(p)) \cap j[\alpha])$. However, $\operatorname{dom}(j(p))=j(\operatorname{dom}(p))=j[\operatorname{dom}(p)]$, which is a subset of $j[\alpha]$. Therefore, the domain of $\pi_{\alpha}(j(p))$ is equal to $j^{-1}(j[\operatorname{dom}(p)])=\operatorname{dom}(p)$. For each $\gamma$ in the domain of $p, \pi_{\alpha}(j(p))(\gamma)=j(p)(j(\gamma)) \uparrow \kappa^{2}=j(p(\gamma)) \uparrow \kappa^{2}$. However, $p(\gamma)$ is a countable subset of $\kappa^{2} \times \omega_{1}$, so $j(p(\gamma))=p(\gamma)$. Since the domain of $p(\gamma)$ is a subset of $\kappa^{2}$, $\pi_{\alpha}(j(p))(\gamma)=j(p(\gamma))\left\lceil\kappa^{2}=p(\gamma)\left\lceil\kappa^{2}=p(\gamma)\right.\right.$, so $\pi_{\alpha}(j(p))=p$.

Since $\pi: j(\mathbb{Q}) \rightarrow \mathbb{Q}$ is a projection mapping, if $\bar{I}$ is a generic filter on $j(\mathbb{Q})$ over $V[\bar{G} * \bar{H}]$, then $\pi[\bar{I}]$ generates a generic filter $I$ on $\mathbb{Q}$ over $V[\bar{G} * \bar{H}]$.

Lemma 11.10. $\pi[\bar{I}]=I$.

Proof. It suffices to show $\pi[\bar{I}]$ is upwards closed. Let $q$ be in $\bar{I}$ and suppose $\pi(q) \leq p$ in $\mathbb{Q}$. By Lemma 11.8, $q \leq j(p)$ in $j(\mathbb{Q})$. Therefore, $j(p)$ is in $\bar{I}$. By Lemma 11.9, $\pi(j(p))=p$, so $p$ is in $\pi[\bar{I}]$. 
The next theorem is an extension of Theorem 6.1.

Theorem 11.11. Let $\bar{I}$ be a generic filter on $j(\mathbb{Q})$ over $V[\bar{G} * \bar{H}]$. Let $I=\pi[\bar{I}]$. Then $j$ can be lifted to $j: V[G * H * I] \rightarrow M[\bar{G} * \bar{H} * \bar{I}]$ such that $j(G * H * I)=\bar{G} * \bar{H} * \bar{I}$.

Proof. It suffices to show $j[I] \subseteq \bar{I}$. Let $p$ be in $I$, and we show $j(p)$ is in $\bar{I}$. Since $p$ is in $I=\pi[\bar{I}]$, fix $q$ in $\bar{I}$ such that $\pi(q)=p$. In particular, $\pi(q) \leq p$. By Lemma $11.8, q \leq j(p)$ in $j(\mathbb{Q})$. As $q$ is in $\bar{I}, j(p)$ is in $\bar{I}$.

\section{Preserving stationary sets}

As usual, fix a cardinal $\kappa$ which is $\kappa^{+}$-supercompact, and assume $2^{\kappa}=\kappa^{+}$. Let $j: V \rightarrow M$ be an elementary embedding with critical point $\kappa$ such that $j(\kappa)>\kappa^{+}$ and $M^{\kappa^{+}} \subseteq M$. Let $\bar{G} * \bar{H} * \bar{I}$ be a generic filter on $j\left(\operatorname{CoLL}\left(\omega_{1},<\kappa\right) * \mathbb{\mathbb { P }}_{\kappa} * \dot{\mathbb{Q}}\right)$ over $V$. Let $G=\bar{G} \cap \operatorname{CoLL}\left(\omega_{1},<\kappa\right), H=\bar{H} \cap \mathbb{P}_{\kappa}$, and $I=\pi[\bar{I}]$. Extend $j$ to $j: V[G * H * I] \rightarrow M[\bar{G} * \bar{H} * \bar{I}]$.

Our goal in this section is to prove the following theorem. After we complete the proof of this result, we will have all the tools necessary to prove the main theorem of the paper.

Theorem 12.1. In $V[G * H * I]$, let $T$ be a stationary subset of $P_{\omega_{1}}(\kappa)$ which is disjoint from $S_{\kappa}$. Then $T$ is a stationary subset of $P_{\omega_{1}}(\kappa)$ in the model $M[\bar{G} * \bar{H} * \bar{I}]$.

By Theorem 6.1, $M[\bar{G} * \bar{H}]$ is equal to $M[\bar{G}][H]\left[H^{\prime}\right]$, where $H^{\prime}$ is a generic filter on $\mathbb{P}\left(\kappa, S_{\kappa}, j(\kappa)\right)$ over $M[\bar{G}][H]$. Let $G^{\prime}=G \cap \operatorname{CoLL}\left(\omega_{1},[\kappa, j(\kappa))\right)$. Then $M[\bar{G}]=M[G]\left[G^{\prime}\right]$, so $M[\bar{G} * \bar{H}]=M[G]\left[G^{\prime}\right][H]\left[H^{\prime}\right]$.

Lemma 12.2. $M[\bar{G} * \bar{H} * \bar{I}]=M[G * H * I]\left[G^{\prime}\right]\left[H^{\prime}\right][\bar{I}]$.

Proof. We already know that

$$
M[\bar{G} * \bar{H} * \bar{I}]=M[G]\left[G^{\prime}\right][H]\left[H^{\prime}\right][\bar{I}] .
$$

Since $\pi: j(\mathbb{Q}) \rightarrow \mathbb{Q}$ is a projection mapping and $I=\pi[\bar{I}]$, we have

$$
M[G]\left[G^{\prime}\right][H]\left[H^{\prime}\right][\bar{I}]=M[G]\left[G^{\prime}\right][H]\left[H^{\prime}\right][I][\bar{I}] .
$$

Therefore,

$$
M[\bar{G} * \bar{H} * \bar{I}]=M[G]\left[G^{\prime}\right][H]\left[H^{\prime}\right][I][\bar{I}] .
$$

Now we repeatedly apply the Product Lemma. Since $\mathbb{P}_{\kappa}$ is in $M[G]$,

$$
M[G]\left[G^{\prime}\right][H]=M[G][H]\left[G^{\prime}\right],
$$

so

$$
M[\bar{G} * \bar{H} * \bar{I}]=M[G][H]\left[G^{\prime}\right]\left[H^{\prime}\right][I][\bar{I}] .
$$

Since $\mathbb{Q}$ is in $M[G][H]$,

$$
M[G][H]\left[G^{\prime}\right]\left[H^{\prime}\right][I]=M[G][H][I]\left[G^{\prime}\right]\left[H^{\prime}\right] .
$$

Therefore,

$$
M[\bar{G} * \bar{H} * \bar{I}]=M[G * H * I]\left[G^{\prime}\right]\left[H^{\prime}\right][\bar{I}] .
$$

Now we begin the (long) proof of Theorem 12.1. 
Proof. Let $T$ be a stationary subset of $P_{\omega_{1}}(\kappa)$ in $V[G * H * I]$ which is disjoint from $S_{\kappa}$. Since $\operatorname{Colv}\left(\omega_{1},<\kappa\right) * \dot{\mathbb{P}}_{\kappa} * \dot{\mathbb{Q}}$ is $\kappa$-c.c., $M[G * H * I]^{\kappa^{+}} \cap V[G * H * I] \subseteq M[G * H * I]$. Hence, $T$ is in $M[G * H * I]$.

By the preceding lemma, $M[\bar{G} * \bar{H} * \bar{I}]$ is equal to $M[G * H * I]\left[G^{\prime}\right]\left[H^{\prime}\right][\bar{I}]$. Since $\operatorname{Colv}\left(\omega_{1},[\kappa, j(\kappa))\right)$ is $\omega_{1}$-closed, and hence proper, $T$ remains a stationary subset of $P_{\omega_{1}}(\kappa)$ in the model $M[G * H * I]\left[G^{\prime}\right]$.

By Corollary 4.8, letting $\alpha=\kappa, A=S_{\kappa}$, and $\beta=j(\kappa)$, we get that $\mathbb{P}\left(\kappa, S_{\kappa}, j(\kappa)\right)$ forces over $M[G * H * I]\left[G^{\prime}\right]$ that $T$ is stationary in $P_{\omega_{1}}(\kappa)$. Thus $T$ is stationary in the model $M[G * H * I]\left[G^{\prime}\right]\left[H^{\prime}\right]$. To show that $T$ is stationary in $M[G * H *$ $I]\left[G^{\prime}\right]\left[H^{\prime}\right][\bar{I}]$, it suffices to show that in the model $M[G * H * I]\left[G^{\prime}\right]\left[H^{\prime}\right]$, the forcing poset $j(\mathbb{Q}) / \mathbb{Q}=\{q \in j(\mathbb{Q}): \pi(q) \in I\}$ forces that $T$ is stationary in $P_{\omega_{1}}(\kappa)$.

Goal: To prove that in the model $M[G * H * I]\left[G^{\prime}\right]\left[H^{\prime}\right]=M[\bar{G} * \bar{H}][I]$, the forcing poset $j(\mathbb{Q}) / \mathbb{Q}$ forces that $T$ is a stationary subset of $P_{\omega_{1}}(\kappa)$.

Before beginning the proof, let us recall some of the notation used in the definition of $\mathbb{Q}$ and fix some additional parameters.

The forcing poset $\mathbb{Q}$ is defined in the model $V[G * H]$, and in this model, $\kappa=\omega_{2}$ and $\kappa^{+}=\omega_{3}$. (As before, we will always write $\kappa^{+}$to denote the ordinal which is the successor of $\kappa$ in the ground model $V$.) In $M[\bar{G} * \bar{H}]$, both $\kappa$ and $\kappa^{+}$are ordinals with cofinality $\omega_{1}$ strictly between $\omega_{1}$ and $j(\kappa)=\omega_{2}$.

Recall Notation 8.4, where we fixed, in $V[G * H]$, a surjective function $g_{\alpha}: \omega_{1} \rightarrow \alpha$ for each $\alpha$ in $\omega_{2}=\kappa$. We let $E$ be the set of $b$ in $P_{\omega_{1}}(\kappa)$ such that ot $(b)$ is a limit ordinal, and for all $\alpha$ in $b, b$ is closed under $g_{\alpha}$. Note that the sequence $\left\langle g_{\alpha}: \alpha \in \kappa\right\rangle$ is in $M[G * H]$.

The definition of $\mathbb{Q}$ involved the sequence of nice names $\left\langle\dot{T}(\alpha): \alpha<\kappa^{+}\right\rangle$. Recall that for all $\alpha<\kappa^{+}, \mathbb{Q}_{\alpha}$ forces that $\dot{T}(\alpha)$ is a subset of $S_{\kappa} \cap E$ which does not reflect to any ordinal in $\kappa$ with cofinality $\omega$. By elementarity, $j(\mathbb{Q})$ is defined in $M[\bar{G} * \bar{H}]$ by the sequence of nice names $j\left(\left\langle\dot{T}(\alpha): \alpha<\kappa^{+}\right\rangle\right)$, which we denote by $\left\langle\dot{T}^{j}(\beta): \beta<j\left(\kappa^{+}\right)\right\rangle$.

By upwards absoluteness, in the model $M[\bar{G} * \bar{H}][I], S_{\kappa}$ is a subset of $P_{\omega_{1}}(\kappa)$ which does not reflect to any ordinal in $\kappa$ with cofinality $\omega_{1}$. Applying Lemma 4.10, fix a club set $D \subseteq P_{\omega_{1}}(\kappa)$ as follows.

Notation 12.3. Let $D$ be a club subset of $P_{\omega_{1}}(\kappa)$ in $M[\bar{G} * \bar{H}][I]$ such that for all $b$ in $D$, for any $\nu$ in $b \cap \operatorname{cof}\left(\omega_{1}\right), b \cap \nu$ is not in $S_{\kappa}$.

For the remainder of the proof, we work in the model $M[\bar{G} * \bar{H}][I]$, unless specified otherwise. We will prove that in this model, the forcing poset $j(\mathbb{Q}) / \mathbb{Q}$ forces that $T$ is a stationary subset of $P_{\omega_{1}}(\kappa)$. To prove this, it suffices to show that whenever $p$ is a condition in $j(\mathbb{Q}) / \mathbb{Q}$ and $p \Vdash \dot{C} \subseteq P_{\omega_{1}}(\kappa)$ is club, then there is $q \leq p$ in $j(\mathbb{Q}) / \mathbb{Q}$ and a set $z$ in $T$ such that $q \Vdash z \in \dot{C}$. Therefore, let $p$ and $\dot{C}$ be given, and assume $p \Vdash \dot{C} \subseteq P_{\omega_{1}}(\kappa)$ is club.

Fix a regular cardinal $\theta$ much larger than $j(\mathbb{Q}) / \mathbb{Q}$ and $\dot{C}$. We use Lemma 4.7 to choose an appropriate elementary substructure of $H(\theta)$. The model we are working in, namely $M[\bar{G} * \bar{H}][I]$, is equal to $M[G * H * I]\left[G^{\prime}\right]\left[H^{\prime}\right]$, which is a generic extension of $M[G * H * I]\left[G^{\prime}\right]$ by the forcing poset $\mathbb{P}\left(\kappa, S_{\kappa}, j(\kappa)\right)$. In $M[G * H * I]\left[G^{\prime}\right], \theta$ is a regular cardinal larger than $\omega_{2}=j(\kappa), \kappa$ has cofinality 
$\omega_{1}$, and $T$ is a stationary subset of $P_{\omega_{1}}(\kappa)$ which is disjoint from $S_{\kappa}$. By Lemma 4.7 , in $M[G * H * I]\left[G^{\prime}\right]\left[H^{\prime}\right]$ there are stationarily many $N$ in $P_{\omega_{1}}(H(\theta))$ such that $S\left(\kappa, S_{\kappa}, j(\kappa)\right) \cap P(N \cap j(\kappa)) \subseteq N$ and $N \cap \kappa \in T$.

Notation 12.4. Let $N$ be a countable subset of $H(\theta)$ in $M[\bar{G} * \bar{H}][I]$ satisfying:

- $N \prec H(\theta)$,

- $N \cap \kappa \in T \cap D$,

- $S\left(\kappa, S_{\kappa}, j(\kappa)\right) \cap P(N \cap j(\kappa)) \subseteq N$,

- $N$ contains as members the objects $j(\mathbb{Q}),\left\langle\dot{T}^{j}(\beta): \beta<j\left(\kappa^{+}\right)\right\rangle, I, j(\mathbb{Q}) / \mathbb{Q}$, $p, \dot{C}, j\left\lceil\kappa^{+}\right.$, and $\left\langle g_{\alpha}: \alpha \in \kappa\right\rangle$.

Since $N \cap \kappa$ is in $T$, it suffices to find $q \leq p$ in $j(\mathbb{Q}) / \mathbb{Q}$ such that $q \Vdash N \cap \kappa \in \dot{C}$. If $q \leq p$ is $N$-generic, then since $\dot{C}$ is in $N, q \Vdash N[\dot{K}] \cap \kappa=N \cap \kappa \in \dot{C}$, where $\dot{K}$ is the canonical $j(\mathbb{Q}) / \mathbb{Q}$-name for the generic filter. Therefore, it suffices to define an $N$-generic sequence $\left\langle p_{n}: n<\omega\right\rangle$ for $j(\mathbb{Q}) / \mathbb{Q}$, with $p_{0}=p$, and to find a lower bound $q$ of this sequence.

We will choose the $N$-generic sequence carefully. Enumerate all dense open subsets of $j(\mathbb{Q}) / \mathbb{Q}$ in $N$ as $\left\langle D_{n}: n<\omega\right\rangle$. By the $\omega_{1}$-distributivity of the forcing posets, the set $N \cap \kappa$ is in $M[G * H]$. Therefore, by Lemma 4.6, $S_{\kappa} \cap P(N \cap \kappa)$ is countable in $M[G * H]$, and hence is countable in $M[\bar{G} * \bar{H}][I]$. Let $\left\langle x_{n}: n<\omega\right\rangle$ be an enumeration of $S_{\kappa} \cap P(N \cap \kappa)$ in which each set appears infinitely often.

Now we define the $N$-generic sequence $\left\langle p_{n}: n<\omega\right\rangle$. Let $p_{0}=p$. Suppose $n<\omega$ and $p_{n}$ is defined. Choose a condition $p_{n}^{*} \leq p_{n}$ which is in $D_{n} \cap N$. Since the set of square conditions in $j(\mathbb{Q})$ is dense in $j(\mathbb{Q})$, the set of square conditions in $j(\mathbb{Q}) / \mathbb{Q}$ is dense in $j(\mathbb{Q}) / \mathbb{Q}$ by Lemma 1.1. Choose a square condition $p_{n}^{\prime} \leq p_{n}^{*}$ in $(j(\mathbb{Q}) / \mathbb{Q}) \cap N$. We split the definition of $p_{n+1}$ into three cases.

Case 1. $x_{n} \subseteq a^{p_{n}^{\prime}}$.

In this case, let $p_{n+1}=p_{n}^{\prime}$.

Case 2. Case 1 fails and $N \cap \omega_{1} \subseteq x_{n}$.

In this case, again let $p_{n+1}=p_{n}^{\prime}$.

Case 3. Case 1 and Case 2 fail.

By the failure of Case $1, x_{n}$ is not a subset of $a^{p_{n}^{\prime}}$. Fix an ordinal $\nu_{n}$ which is in $x_{n} \backslash a^{p_{n}^{\prime}}$. By the failure of Case $2, N \cap \omega_{1}$ is not a subset of $x_{n}$. Fix an ordinal $\zeta_{n}$ in $\left(N \cap \omega_{1}\right) \backslash x_{n}$. Since $\nu_{n}$ is in $x_{n}$ and $\zeta_{n}$ is not in $x_{n}, \nu_{n}$ is not equal to $\zeta_{n}$.

To define $p_{n+1}$, we apply Lemma 9.11. The condition $p_{n}^{\prime}$ is a square condition in $j(\mathbb{Q})$. The ordinal $\nu_{n}$ is in $j(\kappa) \backslash a^{p_{n}^{\prime}}$, and $\zeta_{n}$ is an ordinal in $\omega_{1}$ different from $\nu_{n}$. Let $b=\operatorname{dom}\left(p_{n}^{\prime}\right) \cap j\left[\kappa^{+}\right]$. By Lemma 9.11 , there is a condition $p_{n+1} \leq p_{n}^{\prime}$ in $j(\mathbb{Q})$ such that $\operatorname{dom}\left(p_{n+1}\right)=\operatorname{dom}\left(p_{n}^{\prime}\right)$, for all $\beta$ in $b, p_{n+1}(\beta)=p_{n}^{\prime}(\beta)$, and for all $\beta$ in $\operatorname{dom}\left(p_{n+1}\right) \backslash b, \nu_{n}$ is in $a_{\beta}^{p_{n+1}}$ and $p_{n+1}(\beta)\left(\nu_{n}, \nu_{n}\right)=\zeta_{n}$. Since $j(\mathbb{Q}), p_{n}^{\prime}, b, \nu_{n}$, and $\zeta_{n}$ are all in $N$, by elementarity we can choose $p_{n+1}$ in $N$.

We claim that $p_{n+1}$ is in $j(\mathbb{Q}) / \mathbb{Q}$, that is, $\pi\left(p_{n+1}\right) \in I$. Now $\operatorname{dom}\left(p_{n+1}\right)=$ $\operatorname{dom}\left(p_{n}^{\prime}\right)$ and $p_{n+1}(\beta)=p_{n}^{\prime}(\beta)$ for all $\beta$ in $\operatorname{dom}\left(p_{n}^{\prime}\right) \cap j\left[\kappa^{+}\right]$. It follows that $\pi\left(p_{n+1}\right)=\pi\left(p_{n}^{\prime}\right)$. For $\operatorname{dom}\left(\pi\left(p_{n+1}\right)\right)=j^{-1}\left(\operatorname{dom}\left(p_{n+1}\right) \cap j\left[\kappa^{+}\right]\right)=j^{-1}\left(\operatorname{dom}\left(p_{n}^{\prime}\right) \cap\right.$ $\left.j\left[\kappa^{+}\right]\right)=\operatorname{dom}\left(\pi\left(p_{n}^{\prime}\right)\right)$ and for all $\gamma$ in $\operatorname{dom}\left(\pi\left(p_{n+1}\right)\right), \pi\left(p_{n+1}\right)(\gamma)=p_{n+1}(j(\gamma))$ । $\kappa^{+}=p_{n}^{\prime}(j(\gamma))\left\lceil\kappa^{2}=\pi\left(p_{n}^{\prime}\right)(\gamma)\right.$. Since $\pi\left(p_{n}^{\prime}\right)$ is in $I, \pi\left(p_{n+1}\right)$ is in $I$. Therefore $p_{n+1}$ is in $j(\mathbb{Q}) / \mathbb{Q}$.

This completes the definition of the $N$-generic sequence $\left\langle p_{n}: n<\omega\right\rangle$. 
We define $q$, a lower bound of the sequence $\left\langle p_{n}: n<\omega\right\rangle$, as follows. Let the domain of $q$ be equal to $\bigcup\left\{\operatorname{dom}\left(p_{n}\right): n<\omega\right\}$. If $\beta$ is in the domain of $q$, define $q(\beta)$ as the union of the set of functions $\left\{p_{n}(\beta): n<\omega\right\}$. Clearly $q$ is a partial function $q: j\left(\kappa^{+}\right) \rightarrow j(\mathbb{R})$ with a countable domain. Furthermore, by the $N$-genericity of the sequence $\left\langle p_{n}: n\langle\omega\rangle, \operatorname{dom}(q)=N \cap j\left(\kappa^{+}\right)\right.$, and for each $\beta$ in $\operatorname{dom}(q)$, $a_{\beta}^{q}=N \cap j(\kappa)$.

We will prove that $q$ is in $j(\mathbb{Q}) / \mathbb{Q}$. It then follows easily that $q \leq p_{n}$ for all $n<\omega$, which completes the proof. The proof that $q$ is in $j(\mathbb{Q}) / \mathbb{Q}$ splits into two parts: $(1)$ proving that $q$ is in $j(\mathbb{Q})$, and $(2)$ proving that $\pi(q)$ is in $I$. The second part can be handled quickly, so we take care of it first.

Claim 12.5. Suppose $q$ is in $j(\mathbb{Q})$. Then $\pi(q) \in I$, so $q$ is in $j(\mathbb{Q}) / \mathbb{Q}$.

Proof. Assume $q$ is in $j(\mathbb{Q})$. Then $\pi(q)$ is in $\mathbb{Q}$. As a subclaim, we prove

$$
\operatorname{dom}(\pi(q))=\bigcup\left\{\operatorname{dom}\left(\pi\left(p_{n}\right)\right): n<\omega\right\},
$$

and for all $\gamma$ in $\operatorname{dom}(\pi(q))$,

$$
\pi(q)(\gamma)=\bigcup\left\{\pi\left(p_{n}\right)(\gamma): n<\omega\right\} .
$$

We have that $\operatorname{dom}(\pi(q))=j^{-1}\left(\operatorname{dom}(q) \cap j\left[\kappa^{+}\right]\right)=j^{-1}\left(\left(\bigcup\left\{\operatorname{dom}\left(p_{n}\right): n<\omega\right\}\right) \cap\right.$ $\left.j\left[\kappa^{+}\right]\right)=j^{-1}\left(\bigcup\left\{\operatorname{dom}\left(p_{n}\right) \cap j\left[\kappa^{+}\right]: n<\omega\right\}\right)=\bigcup\left\{j^{-1}\left(\operatorname{dom}\left(p_{n}\right) \cap j\left[\kappa^{+}\right]\right): n<\omega\right\}=$ $\bigcup\left\{\operatorname{dom}\left(\pi\left(p_{n}\right)\right): n<\omega\right\}$. Now let $\gamma$ be in $\operatorname{dom}(\pi(q))$. Then $\pi(q)(\gamma)=q(j(\gamma)) \uparrow$ $\kappa^{2}=\left(\bigcup\left\{p_{n}(j(\gamma)): n<\omega\right\}\right)\left\lceil\kappa^{2}=\bigcup\left\{p_{n}(j(\gamma))\left\lceil\kappa^{2}: n<\omega\right\}=\bigcup\left\{\pi\left(p_{n}\right)(\gamma): n<\right.\right.\right.$ $\omega\}$.

For each $n<\omega, p_{n}$ is in $j(\mathbb{Q}) / \mathbb{Q}$, and so $\pi\left(p_{n}\right)$ is in $I$. Hence $\left\langle\pi\left(p_{n}\right): n<\omega\right\rangle$ is a descending sequence of conditions in $I$. By Lemma 9.10, there is $r$ in $I$ such that $r \leq \pi\left(p_{n}\right)$ for all $n<\omega$. It follows from the subclaim that $\operatorname{dom}(\pi(q)) \subseteq \operatorname{dom}(r)$, and for all $\gamma$ in $\operatorname{dom}(\pi(q)), r(\gamma)\left\lceil a_{\gamma}^{\pi(q)}=\pi(q)(\gamma)\right.$. Thus $r \leq \pi(q)$ in $\mathbb{Q}$. Since $r$ is in $I$ and $I$ is a filter, $\pi(q)$ is in $I$.

It remains to show that $q$ is in $j(\mathbb{Q})$. We defined the condition $q$ in the model $M[\bar{G} * \bar{H}][I]$, whereas the forcing poset $j(\mathbb{Q})$ is defined in $M[\bar{G} * \bar{H}]$. But $\mathbb{Q}$ is $\omega_{1}$-distributive in $M[\bar{G} * \bar{H}]$. This is true since $j(\mathbb{Q})$ is $\omega_{1}$-distributive in $M[\bar{G} * \bar{H}]$ and $\pi: j(\mathbb{Q}) \rightarrow \mathbb{Q}$ is a projection mapping. It follows that $q$ is in $M[\bar{G} * \bar{H}]$. We will prove in the model $M[\bar{G} * \bar{H}]$ that $q$ is in $j(\mathbb{Q})$.

Suppose for a contradiction that $q$ is not in $j(\mathbb{Q})$. Applying Lemma 9.3 in the model $M[\bar{G} * \bar{H}]$, we can fix $\beta$, $t$, and $x$ such that:

- $\beta$ is in $\operatorname{dom}(q)$,

- $q\left\lceil\beta\right.$ is in $j(\mathbb{Q})_{\beta}$,

- $t \leq q \uparrow \beta$ in $j(\mathbb{Q})_{\beta}$,

- $x$ is a countable subset of $a_{\beta}^{q}$ closed under $q(\beta)$,

- $t \Vdash_{j(\mathbb{Q})_{\beta}} x \in \dot{T}^{j}(\beta)$.

Recall that $\Vdash_{j(\mathbb{Q})_{\beta}} \dot{T}^{j}(\beta) \subseteq j\left(S_{\kappa}\right) \cap j(E)$. Therefore, since $t \Vdash_{j(\mathbb{Q})_{\beta}} x \in \dot{T}^{j}(\beta), x$ is in $j\left(S_{\kappa}\right) \cap j(E)$. By Theorem 6.1, $j\left(S_{\kappa}\right)=S_{j(\kappa)}=S_{\kappa} \cup S\left(\kappa, S_{\kappa}, j(\kappa)\right)$. Therefore, there are two possibilities concerning $x$ : either $x \subseteq \kappa$ and $x \in S_{\kappa}$, or $x \nsubseteq \kappa$ and $x \in S\left(\kappa, S_{\kappa}, j(\kappa)\right)$.

Note that $\beta$ is in $N$, since $\operatorname{dom}(q)=N \cap j\left(\kappa^{+}\right)$. Since $q \uparrow \beta$ is in $j(\mathbb{Q})_{\beta}$, it is easy to see that $q \uparrow \beta \leq p_{n} \uparrow \beta$ for all $n<\omega$. In particular, $q \uparrow \beta$ is $N$-generic for $j(\mathbb{Q})_{\beta}$. 
Claim 12.6. For all $n<\omega$, if $\beta \in \operatorname{dom}\left(p_{n}\right)$, then $x$ is not a subset of $a_{\beta}^{p_{n}}$.

Proof. Suppose $x$ is a subset of $a_{\beta}^{p_{n}}$. Since $q(\beta)$ extends $p_{n}(\beta)$ as a function, $x$ is closed under $p_{n}(\beta)$. By Lemma 9.2, $p_{n}\left\lceil\beta \Vdash_{j(\mathbb{Q})_{\beta}} x \notin \dot{T}^{j}(\beta)\right.$. However, $t \leq q\lceil\beta \leq$ $p_{n}\left\lceil\beta\right.$, so $t \Vdash_{j(\mathbb{Q})_{\beta}} x \notin \dot{T}^{j}(\beta)$, contradicting the choice of $t$.

Claim 12.7. The set $x$ is not in $N$.

Proof. Let $D$ be the dense open set of conditions $s$ in $j(\mathbb{Q}) / \mathbb{Q}$ such that $\beta$ is in $\operatorname{dom}(s)$ and $x \subseteq a_{\beta}^{s}$. If $x$ is in $N$, then by elementarity, $D$ is in $N$. By $N$-genericity, fix $n<\omega$ such that $p_{n}$ is in $D$. Then $x \subseteq a_{\beta}^{p_{n}}$, contradicting the last claim.

Claim 12.8. The set $x$ is in $S_{\kappa}$.

Proof. If $x$ is not in $S_{\kappa}$, then $x$ is in $S\left(\kappa, S_{\kappa}, j(\kappa)\right)$. Since $q(\beta)=\bigcup\left\{p_{n}(\beta): n<\omega\right\}$, $a_{\beta}^{q}=\bigcup\left\{a_{\beta}^{p_{n}}: n<\omega\right\}$, which by $N$-genericity is equal to $N \cap j(\kappa)$. Since $x \subseteq a_{\beta}^{q}$, $x$ is a subset of $N \cap j(\kappa)$. Therefore $x$ is in $S\left(\kappa, S_{\kappa}, j(\kappa)\right) \cap P(N \cap j(\kappa))$. By the choice of $N$, as described in Notation 12.4, $S\left(\kappa, S_{\kappa}, j(\kappa)\right) \cap P(N \cap j(\kappa)) \subseteq N$. Thus $x$ is in $N$. This contradicts Claim 12.7 .

Therefore, $x$ is a subset of $\kappa$. Also, $x$ is a subset of $a_{\beta}^{q}$, which is equal to $N \cap j(\kappa)$, so $x$ is a subset of $N \cap \kappa$.

Let $j\left(\left\langle g_{\alpha}: \alpha<\kappa\right\rangle\right)=\left\langle g_{\delta}^{j}: \delta<j(\kappa)\right\rangle$. For all $\alpha<\kappa, g_{\alpha}^{j}=g_{j(\alpha)}^{j}=j\left(g_{\alpha}\right)$, which is equal to $g_{\alpha}$, since $g_{\alpha}$ is a subset of $\omega_{1} \times \alpha$. Since $x$ is in $j(E)$, it follows that for all $\alpha$ in $x, x$ is closed under $g_{\alpha}$.

Claim 12.9. The ordinal $\beta$ is not in $j\left[\kappa^{+}\right]$.

Proof. Suppose for a contradiction that $\beta$ is in $j\left[\kappa^{+}\right]$. Let $\gamma=j^{-1}(\beta)$, so $j(\gamma)=\beta$. Then $\gamma$ is in $j^{-1}\left(\operatorname{dom}(q) \cap j\left[\kappa^{+}\right]\right)=\operatorname{dom}(\pi(q))$.

By Claim 12.8, $x \subseteq \kappa$. Since $q(\beta)\left\lceil\kappa^{2}=\pi(q)(\gamma), x\right.$ is a countable subset of $a_{\gamma}^{\pi(q)}$ which is closed under $\pi(q)(\gamma)$. Since $M[\bar{G} * \bar{H}]$ is a generic extension of $M$ by an $\omega_{1}$-distributive forcing poset, $x$ is in $M$ and hence is in $V[G * H]$. Applying Lemma 9.2 in $V[G * H], \pi(q)\left\lceil\gamma \Vdash_{\mathbb{Q}_{\gamma}} x \notin \dot{T}(\gamma)\right.$.

By Lemma 11.4, $\pi_{\gamma}(q \uparrow \beta)=\pi(q) \uparrow \gamma$, so $\pi_{\gamma}(q \uparrow \beta) \Vdash_{\mathbb{Q}_{\gamma}} x \notin \dot{T}(\gamma)$. Now $t \leq q \uparrow \beta$, so $\pi_{\gamma}(t) \leq \pi_{\gamma}\left(q\lceil\beta)\right.$. Hence $\pi_{\gamma}(t) \Vdash_{\mathbb{Q}_{\gamma}} x \notin \dot{T}(\gamma)$. Since $j(x)=x, j\left(\mathbb{Q}_{\gamma}\right)=j(\mathbb{Q})_{\beta}$, and $j(\dot{T}(\gamma))=\dot{T}^{j}(\beta)$, by the elementarity of $j$ we have $j\left(\pi_{\gamma}(t)\right) \Vdash_{j(\mathbb{Q})_{\beta}} x \notin \dot{T}^{j}(\beta)$. On the other hand, $t \Vdash_{j(\mathbb{Q})_{\beta}} x \in \dot{T}^{j}(\beta)$. But by Proposition 11.7, $j\left(\pi_{\gamma}(t)\right)$ and $t$ are compatible in $j(\mathbb{Q})_{\beta}$, which is a contradiction.

We split the remainder of the proof into two cases. The first case is that $x$ is an initial segment of $N \cap \kappa$, and the second case is that $x$ is not an initial segment of $N \cap \kappa$. We will derive a contradiction in both cases. This shows that $q$ is indeed a condition in $j(\mathbb{Q})$, which completes the proof.

Case a. $x$ is an initial segment of $N \cap \kappa$.

Note that $x$ cannot be equal to $N \cap \kappa$ itself. For by the choice of $N$, as described in Notation 12.4, $N \cap \kappa \in T$. But $x$ is in $S_{\kappa}$ and $T$ is disjoint from $S_{\kappa}$.

Let $\nu$ be the least ordinal in $N \cap \kappa$ which is not in $x$. Then $x=N \cap \nu$. Recall that since $t \Vdash_{j(\mathbb{Q})_{\beta}} x \in \dot{T}^{j}(\beta), x$ is in $j\left(S_{\kappa}\right) \cap j(E)$. By the definition of $E$, this implies ot $(x)$ is a limit ordinal. It follows that $\nu$ is a limit ordinal. 
Recall by Notation 12.3 that $D$ is a club subset of $P_{\omega_{1}}(\kappa)$ in $M[\bar{G} * \bar{H}][I]$ such that for all $b$ in $D$, if $\gamma$ is in $b \cap \operatorname{cof}\left(\omega_{1}\right)$, then $b \cap \gamma$ is not in $S_{\kappa}$. By the choice of $N$, as described in Notation 12.4, $N \cap \kappa$ is in $D$. Since $N \cap \nu=x$ is in $S_{\kappa}, \nu$ is not in $(N \cap \kappa) \cap \operatorname{cof}\left(\omega_{1}\right)$. Therefore, $\operatorname{cf}(\nu)=\omega$.

In the model $M[\bar{G} * \bar{H}]$, the forcing poset $j(\mathbb{Q})_{\beta}$ forces that $\dot{T}^{j}(\beta)$ does not reflect to any ordinal in $j(\kappa)$ with cofinality $\omega$. As $\nu$ has cofinality $\omega$, we can fix a $j(\mathbb{Q})_{\beta^{-}}$ name $\dot{F}_{\nu}$ for a function $\dot{F}_{\nu}:[\nu]^{<\omega} \rightarrow \nu$ such that any $b$ in $\dot{T}^{j}(\beta)$ which is a subset of $\nu$ is not closed under $\dot{F}_{\nu}$. Since $j(\mathbb{Q}), \beta, \dot{T}^{j}(\beta)$, and $\nu$ are in $N$, by elementarity we can choose such a name $\dot{F}_{\nu}$ in $N$.

As $\dot{F}_{\nu}$ is in $N, j(\mathbb{Q})_{\beta}$ forces that $N[\dot{K}] \cap \nu$ is closed under $\dot{F}_{\nu}$, where $\dot{K}$ is the canonical $j(\mathbb{Q})_{\beta}$-name for a generic filter. Recall that $q \uparrow \beta \leq p_{n} \uparrow \beta$ for all $n<\omega$, and so $q \uparrow \beta$ is $N$-generic for $j(\mathbb{Q})_{\beta}$. Since $t \leq q \uparrow \beta$, $t$ is also $N$-generic, and so $t$ forces $N[\dot{K}] \cap \nu=N \cap \nu=x$ is closed under $\dot{F}_{\nu}$. However, $t \Vdash_{j(\mathbb{Q})_{\beta}} x \in \dot{T}^{j}(\beta)$, contradicting the choice of $\dot{F}_{\nu}$.

Case b. $x$ is not an initial segment of $N \cap \kappa$.

Fix ordinals $\gamma<\alpha<\kappa$ such that $\gamma$ is in $N \backslash x$ and $\alpha$ is in $x$. Recall that $g_{\alpha}$ is a surjection of $\omega_{1}$ onto $\alpha$. Since $\alpha$ is in $N, g_{\alpha}$ is in $N$. As $\gamma$ is in $N \cap \alpha$, by elementarity fix $\xi$ in $N \cap \omega_{1}$ such that $g_{\alpha}(\xi)=\gamma$. Since $\alpha$ is in $x, x$ is closed under $g_{\alpha}$. Therefore $\xi$ is not in $x$, since otherwise $g_{\alpha}(\xi)=\gamma$ would be in $x$.

Recall that $\left\langle x_{n}: n<\omega\right\rangle$ is an enumeration of $S_{\kappa} \cap P(N \cap \kappa)$ in which each set appears infinitely often. However, $x$ is in $S_{\kappa} \cap P(N \cap \kappa)$. Since $\beta$ is in $\bigcup\left\{\operatorname{dom}\left(p_{n}\right)\right.$ : $n<\omega\}$, choose $n$ large enough so that $\beta$ is in $\operatorname{dom}\left(p_{n}\right)$ and $x_{n}=x$.

Now recall the three cases in the definition of $p_{n+1}$. The first case is that $x_{n} \subseteq$ $a_{\beta}^{p_{n}^{\prime}}$. This is false by Claim 12.6. The second case is that $N \cap \omega_{1} \subseteq x_{n}$. This is false, since $\xi$ is in $N \cap \omega_{1}$ but not in $x$. Hence, we are in the third case in the definition of $p_{n+1}$. In that case we fixed an ordinal $\nu_{n}$ which is in $x_{n} \backslash a^{p_{n}^{\prime}}$ and an ordinal $\zeta_{n}$ which is in $\left(N \cap \omega_{1}\right) \backslash x_{n}$. Then we chose $p_{n+1}$ so that for all $\delta$ in $\operatorname{dom}\left(p_{n+1}\right) \backslash j\left[\kappa^{+}\right], \nu_{n}$ is in $a_{\delta}^{p_{n+1}}$ and $p_{n+1}(\delta)\left(\nu_{n}, \nu_{n}\right)=\zeta_{n}$. By Claim 12.9, $\beta$ is in $\operatorname{dom}\left(p_{n+1}\right) \backslash j\left[\kappa^{+}\right]$. Therefore $\nu_{n}$ is in $a_{\beta}^{p_{n+1}}$ and $p_{n+1}(\beta)\left(\nu_{n}, \nu_{n}\right)=\zeta_{n}$. It follows that $q(\beta)\left(\nu_{n}, \nu_{n}\right)=\zeta_{n}$. Since $\nu_{n}$ is in $x_{n}$ and $\zeta_{n}$ is not in $x_{n}, x_{n}$ is not closed under $q(\beta)$. However, $x=x_{n}$, and $x$ was assumed to be closed under $q(\beta)$. Thus we have reached our final contradiction.

\section{THE MAIN THEOREM}

We now have all the tools necessary to complete the proof of the main theorem of the paper.

Theorem 13.1. Suppose $\kappa$ is a $\kappa^{+}$-supercompact cardinal and $2^{\kappa}=\kappa^{+}$. Then there is a forcing poset $\mathbb{P}$, namely,

$$
\operatorname{CoLL}\left(\omega_{1},<\kappa\right) * \dot{\mathbb{P}}_{\kappa} * \dot{\mathbb{Q}},
$$

such that

$$
\Vdash_{\mathbb{P}} W R P\left(\omega_{2}\right) \wedge \neg R P\left(\omega_{2}\right) .
$$

Proof. Let $\mathbb{P}$ denote $\operatorname{ColL}\left(\omega_{1},<\kappa\right) * \dot{\mathbb{P}}_{\kappa} * \dot{\mathbb{Q}}$. Then $\mathbb{P}$ is $\omega_{1}$-distributive and $\omega_{2^{-}}$ c.c. Let $j: V \rightarrow M$ be an elementary embedding with critical point $\kappa$ such that $j(\kappa)>\kappa^{+}$and $M^{\kappa^{+}} \subseteq M$. 
Let $\varphi$ be the statement $\operatorname{WRP}\left(\omega_{2}\right) \wedge \neg \mathrm{RP}\left(\omega_{2}\right)$. To show $\Vdash_{\mathbb{P}} \varphi$, it suffices to show that whenever $\bar{G} * \bar{H} * \bar{I}$ is a generic filter on $j(\mathbb{P})$ over $V$, then letting $G=\bar{G} \cap \operatorname{CoLL}\left(\omega_{1},<\kappa\right), H=\bar{H} \cap \mathbb{P}_{\kappa}$, and $I=\pi[\bar{I}], V[G * H * I] \models \varphi$. For if $\Vdash_{\mathbb{P}} \varphi$ is false, then there is a condition $p * \dot{q} * \dot{r}$ in $\mathbb{P}$ such that $p * \dot{q} * \dot{r} \Vdash_{\mathbb{P}} \neg \varphi$. Choose $\bar{G} * \bar{H} * \bar{I}$ which contains the condition $j(p * \dot{q} * \dot{r})$. By Theorems 6.1 and 11.11, extend $j$ to $j: V[G * H * I] \rightarrow M[\bar{G} * \bar{H} * \bar{I}]$ such that $j(G * H * I)=\bar{G} * \bar{H} * \bar{I}$. Since $j(p)=p$ is in $\bar{G}=j(G), p$ is in $G$. As $j(q)=q$ is in $\bar{H}=j(H), q$ is in $H$. By Lemma $11.9, \pi(j(r))=r$ is in $\pi[\bar{I}]=I$. Therefore, $p * \dot{q} * \dot{r}$ is in $G * H * I$, and thus $V[G * H * I] \models \neg \varphi$.

Let $\bar{G} * \bar{H} * \bar{I}$ be a generic filter on $j(\mathbb{P})$ over $V$, and let $G, H$, and $I$ be as above. We prove $V[G * H * I] \models \varphi$. By Theorems 6.1 and 11.11 , we can extend $j$ to $j: V[G * H * I] \rightarrow M[\bar{G} * \bar{H} * \bar{I}]$ such that $j(G * H * I)=\bar{G} * \bar{H} * \bar{I}$. By Theorem $10.2, S_{\kappa}$ is a stationary subset of $P_{\omega_{1}}\left(\omega_{2}\right)$ in $V[G * H * I]$ which does not reflect to any ordinal in $\omega_{2}$ with cofinality $\omega_{1}$. Therefore, $V[G * H * I] \models \neg \mathrm{RP}\left(\omega_{2}\right)$.

It remains to show that $V[G * H * I] \models \mathrm{WRP}\left(\omega_{2}\right)$. Let $U$ be a stationary subset of $P_{\omega_{1}}\left(\omega_{2}\right)$ in $V[G * H * I]$. First assume $U \cap S_{\kappa}$ is stationary. Then by Theorem 10.2, there is an uncountable ordinal $\nu$ in $\omega_{2}$ with cofinality $\omega$ such that $\left(U \cap S_{\kappa}\right) \cap P_{\omega_{1}}(\nu)$ is stationary in $P_{\omega_{1}}(\nu)$. Therefore, $U$ reflects to $\nu$.

Now assume $U \cap S_{\kappa}$ is non-stationary. Let $T=U \backslash S_{\kappa}$. Then $T$ is a stationary subset of $P_{\omega_{1}}\left(\omega_{2}\right)$ in $V[G * H * I]$ which is disjoint from $S_{\kappa}$. By Theorem 12.1, T is a stationary subset of $P_{\omega_{1}}(\kappa)$ in $M[\bar{G} * \bar{H} * \bar{I}]$. For any $b$ in $T, j(b)=b$ is in $j(T)$ by the elementarity of $j$, so $T \subseteq j(T) \cap P_{\omega_{1}}(\kappa)$ in $M[\bar{G} * \bar{H} * \bar{I}]$ (in fact, these sets are equal). It follows that $j(T) \cap P_{\omega_{1}}(\kappa)$ is stationary in $P_{\omega_{1}}(\kappa)$ in $M[\bar{G} * \bar{H} * \bar{I}]$. Therefore, $M[\bar{G} * \bar{H} * \bar{I}]$ models the statement that $j(T)$ reflects to an ordinal in $\omega_{2}$ with cofinality $\omega_{1}$. By the elementarity of $j, V[G * H * I]$ models the statement that $T$ reflects to an ordinal in $\omega_{2}$ with cofinality $\omega_{1}$. Since $T \subseteq U, U$ reflects to an uncountable ordinal in $\omega_{2}$.

\section{REFERENCES}

[1] M. Foreman, M. Magidor, and S. Shelah, Martin's maximum, saturated ideals and non-regular ultrafilters I, Ann. of Math. 127 (1988), 1-47. MR924672 (89f:03043)

[2] B. Koenig, P. Larson, and Y. Yoshinobu, Guessing clubs in the generalized club-filter, Fund. Math. 195 (2007), no. 2, 177-191. MR2320769 (2008f:03064)

[3] H. Sakai, Partial stationary reflection principle in $P_{\omega_{1}}\left(\omega_{2}\right)$, Axiomatic Set Theory and SetTheoretic Topology, RIMS Kokyuroku, no. 1595, 2008, pp. 47-62.

[4] S. Shelah, Around classification theory of models, Lecture Notes of Mathematics, vol. 1182, Springer-Verlag, Berlin, 1986. MR850051 (90a:03037)

[5] _ Diamonds, Proc. Amer. Math. Soc. 138 (2010), no. 6, 2151-2161. MR2596054

[6] S. Todorcevic, Reflecting stationary sets, Handwritten Notes, 1985.

[7] B. Veličković, Forcing axioms and stationary sets, Adv. Math. 94 (1992), no. 2, 256-284. MR:1174395 (93k:03045)

Department of Mathematics, University of North Texas, 1155 Union Circle \#311430, Denton, Texas 76203

E-mail address: jkrueger@unt.edu 